\title{
DG MANIFOLDS, FORMAL EXPONENTIAL MAPS AND HOMOTOPY LIE ALGEBRAS
}

\author{
SEOKBONG SEOL, MATHIEU STIÉNON, AND PING XU
}

\begin{abstract}
This paper is devoted to the study of the relation between 'formal exponential maps,' the Atiyah class, and Kapranov $L_{\infty}[1]$ algebras associated with dg manifolds in the $C^{\infty}$ context. We prove that, for a dg manifold, a 'formal exponential map' exists if and only if the Atiyah class vanishes. Inspired by Kapranov's construction of a homotopy Lie algebra associated with the holomorphic tangent bundle of a complex manifold, we prove that the space of vector fields on a dg manifold admits an $L_{\infty}$ [1] algebra structure, unique up to isomorphism, whose unary bracket is the Lie derivative w.r.t. the homological vector field, whose binary bracket is a 1-cocycle representative of the Atiyah class, and whose higher multibrackets can be computed by a recursive formula. For the dg manifold $\left(T_{X}^{0,1}[1], \bar{\partial}\right)$ arising from a complex manifold $X$, we prove that this $L_{\infty}[1]$ algebra structure is quasi-isomorphic to the standard $L_{\infty}[1]$ algebra structure on the Dolbeault complex $\Omega^{0, \bullet}\left(T_{X}^{1,0}\right)$.
\end{abstract}

\section{Contents}

1. Introduction 2

2. Preliminaries 6

2.1. dg manifolds $\quad 6$

2.2. Atiyah class $\quad 8$

3. Formal exponential map of dg manifolds $\quad 9$

$\begin{array}{ll}\text { 3.1. dg coalgebras } & 9\end{array}$

3.2. Formal exponential map of a dg manifold 11

3.3. Proof of Proposition $3.7 \quad 13$

4. Atiyah class and homotopy Lie algebras 16

4.1. Kapranov $L_{\infty}[1]$ algebras of dg manifolds 16

4.2. Recursive formula for multibrackets 20

5. Examples 24

5.1. dg manifolds associated to $L_{\infty}[1]$ algebras 24

5.2. dg manifolds associated to complex manifolds and integrable distributions 30

Appendix A. Fedosov construction on graded manifolds 34

$\begin{array}{ll}\text { Acknowledgments } & 36\end{array}$

$\begin{array}{ll}\text { References } & 36\end{array}$

Research partially supported by NSF grants DMS-1707545 and DMS-2001599. 


\section{INTRODUCTION}

This paper, which is a sequel to [38], is devoted to the study of some differential geometric aspects of dg manifolds in the $C^{\infty}$ context. Dg manifolds (a.k.a. $Q$-manifolds $[1,39,46]$ ) have increasingly attracted attention recently due to their relevance in various fields of mathematics, in particular, mathematical physics. They first appeared in the mathematical physics literature in the study of BRST operators used to describe gauge symmetries. They play an essential role in the so called AKSZ formalism in the study of sigma model quantum field theories $[1,13]$. They arise naturally in a variety of situations in differential geometry, Lie theory, representation theory and homotopy algebras $[28,56,54,55,21]$. They are closely related to the emerging fields of derived differential geometry $[5,11,12,24,40,41,50]$ and higher Lie algebroids $[4,7,8,9,20,22,23,44,56,54,48]$ (see also [47, Letters 7 and 8]).

Recall that a $\boldsymbol{d g}$ manifold is a $\mathbb{Z}$-graded manifold $\mathcal{M}$ endowed with a homological vector field, i.e. a degree +1 derivation $Q$ of $C^{\infty}(\mathcal{M})$ satisfying $[Q, Q]=0$. When the underlying $\mathbb{Z}$-graded manifold $\mathcal{M}$ is a $\mathbb{Z}$-graded vector space, a dg manifold is equivalent to a finite dimensional curved $L_{\infty}$ algebra (or more precisely a curved $L_{\infty}$ [1] algebra). Any complex manifold naturally gives rise to a dg manifold, as does any foliation of a smooth manifold. See Examples 2.2 and 2.3.

The exponential map plays an important role in classical differential geometry. In graded geometry, it turns out that a certain 'formal exponential map' is more useful. Let us describe examples, which illustrate the concept of 'formal exponential map' we have in mind. First of all, let us recall the relation between exponential map and Poincaré-Birkhoff-Witt isomorphism (PBW isomorphism in short) in classical Lie theory. Let $G$ be a Lie group and let $\mathfrak{g}$ be its Lie algebra. The space $D_{0}^{\prime}(\mathfrak{g})$ of distributions on $\mathfrak{g}$ with support $\{0\}$ is canonically identified with the symmetric tensor algebra $S(\mathfrak{g})$, while the space $D_{e}^{\prime}(G)$ of distributions on $G$ with support $\{e\}$ is canonically identified with the universal enveloping algebra $\mathcal{U}(\mathfrak{g})$. The classical Lie-theoretic exponential map exp $: \mathfrak{g} \rightarrow G$, which is a local diffeomorphism near 0 , can be used to push forward the distributions on the Lie algebra to distributions on the Lie group. The induced isomorphism $S(\mathfrak{g}) \cong D_{0}^{\prime}(\mathfrak{g}) \stackrel{\sim}{\rightarrow} D_{e}^{\prime}(G) \cong \mathcal{U}(\mathfrak{g})$ is precisely the symmetrization map realizing the well known PBW isomorphism. This construction has an analogue for smooth manifolds. However, it requires a choice of affine connection. Given a smooth manifold $M$, let $R$ denote its algebra of smooth real-valued functions $C^{\infty}(M)$. Each affine connection $\nabla$ on $M$ determines an exponential map

$$
\exp ^{\nabla}: T_{M} \rightarrow M \times M
$$

which is a local diffeomorphism of fiber bundles

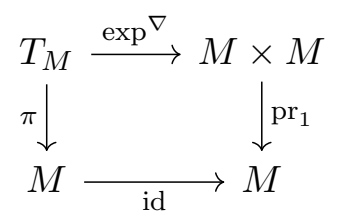

from a neighborhood of the zero section of $T_{M}$ to a neighborhood of the diagonal $\Delta$ in $M \times M$. The space of fiberwise distributions on the vector bundle $\pi: T_{M} \rightarrow M$ with support the zero section can be identified, as an $R$-coalgebra, to $\Gamma\left(S\left(T_{M}\right)\right)$. On the other hand, the space of fiberwise distributions on the fiber bundle $\operatorname{pr}_{1}: M \times M \rightarrow M$ with support the diagonal $\Delta$ can be identified, as an $R$-coalgebra, to the space $\mathcal{D}(M)$ of differential operators on $M$. Pushing distributions forward through the exponential map (1), we obtain an isomorphism of $R$-coalgebras

$$
\mathrm{pbw}^{\nabla}: \Gamma\left(S\left(T_{M}\right)\right) \rightarrow \mathcal{D}(M)
$$


called PBW map in $[30,31]$. In other words, $\mathrm{pbw}^{\nabla}$ is the fiberwise $\infty$-order jet (along the zero section) of the exponential map (1) arising from the connection $\nabla$. Therefore, one can consider it as a 'formal exponential map' associated with the affine connection $\nabla$.

We have the following explicit formula for $\mathrm{pbw}^{\nabla}$ :

$$
\operatorname{pbw}^{\nabla}\left(X_{0} \odot \cdots \odot X_{k}\right)(f)=\left.\left.\left.\frac{d}{d t_{0}}\right|_{0} \frac{d}{d t_{1}}\right|_{0} \cdots \frac{d}{d t_{k}}\right|_{0} f\left(\exp \left(t_{0} X_{0}+t_{1} X_{1}+\cdots+t_{k} X_{k}\right)\right),
$$

for all $X_{0}, X_{1}, \cdots, X_{k} \in \Gamma\left(T_{M}\right)$ and $f \in C^{\infty}(M)$.

It turns out that the map pbw ${ }^{\nabla}$ admits a nice recursive characterization which can be described in a purely algebraic way $[30,31]$ involving the connection $\nabla$, but not the exponential map (1). Therefore, despite the geometric origin of the map $\mathrm{pbw}^{\nabla}$, this 'formal exponential map' still makes sense algebraically in a much wider context. By way of this purely algebraic description, the 'formal exponential map' was extended to the context of $\mathbb{Z}$-graded manifolds over the field $\mathbb{K}$ (with $\mathbb{K}=\mathbb{R}$ or $\mathbb{C}$ ) by Liao-Stiénon [32]. The PBW map:

$$
\operatorname{pbw}^{\nabla}: \Gamma\left(S\left(T_{\mathcal{M}}\right)\right) \rightarrow \mathcal{D}(\mathcal{M})
$$

arising from an affine connection $\nabla$ on a $\mathbb{Z}$-graded manifold $\mathcal{M}$ can be thought of as the induced formal exponential map (or the fiberwise $\infty$-order jet) of a 'virtual exponential map:'

$$
\exp ^{\nabla}: T_{\mathcal{M}} \rightarrow \mathcal{M} \times \mathcal{M}
$$

Now, let $(\mathcal{M}, Q)$ be a dg manifold. Then, both $\Gamma\left(S\left(T_{\mathcal{M}}\right)\right)$ and $\mathcal{D}(\mathcal{M})$ in $(2)$ are $\mathrm{dg}$ coalgebras over the dg ring $\left(C^{\infty}(\mathcal{M}), Q\right)$ - see Propositions 3.2 and 3.3. Here $\left(\Gamma\left(S\left(T_{\mathcal{M}}\right)\right), \mathcal{L}_{Q}\right)$ can be understood as the space of fiberwise dg distributions on the $\mathrm{dg}$ vector bundle $\pi: T_{\mathcal{M}} \rightarrow \mathcal{M}$ with support the zero section - the homological vector field on $T_{\mathcal{M}}$ is the complete lift $\hat{Q}$ of the homological vector field $Q \in \mathfrak{X}(\mathcal{M})[38,51]$. On the other hand, $\left(\mathcal{D}(\mathcal{M}), \mathcal{L}_{Q}^{\mathcal{D}}\right)$ can be identified with the space of fiberwise $\mathrm{dg}$ distributions on the $\mathrm{dg}$ fiber bundle $\mathrm{pr}_{1}: \mathcal{M} \times \mathcal{M} \rightarrow \mathcal{M}$ with support the diagonal $\Delta \in \mathcal{M} \times \mathcal{M}$ - the homological vector field on $\mathcal{M} \times \mathcal{M}$ is $(Q, Q)$. Recall that for an ordinary smooth manifold $M$, equipped with a vector field $Q$, the exponential map (1) arising from a choice of affine connection $\nabla$ on $M$ identifies the complete $\operatorname{lift}^{1} \hat{Q} \in \mathfrak{X}\left(T_{M}\right)$ of $Q \in \mathfrak{X}(M)$ with the vector field $(Q, Q) \in \mathfrak{X}(M \times M)$ if and only if the connection $\nabla$ is invariant under the flow of $Q$. In the similar fashion, one may wonder whether the 'virtual exponential map' (3) is a morphism of dg manifolds. On the level of fiberwise $\infty$-order jets, this is equivalent to asking whether the map $\operatorname{pbw}^{\nabla}:\left(\Gamma\left(S\left(T_{\mathcal{M}}\right)\right), \mathcal{L}_{Q}\right) \rightarrow\left(\mathcal{D}(\mathcal{M}), \mathcal{L}_{Q}^{\mathcal{D}}\right)$ is an isomorphism of dg coalgebras over $\left(C^{\infty}(\mathcal{M}), Q\right)$. As in classical geometry, one expects that this would be true if the affine connection $\nabla$ on $\mathcal{M}$ is invariant under the (virtual) flow of the homological vector field $Q$; in other words, if the Atiyah class of the dg manifold $(\mathcal{M}, Q)$ vanishes.

Our first main theorem confirms this assertion:

Theorem A (Theorem 3.5). Let $(\mathcal{M}, Q)$ be a dg manifold. The Atiyah class $\alpha_{(\mathcal{M}, Q)}$ vanishes if and only if there exists a torsion-free affine connection $\nabla$ on $\mathcal{M}$ such that

$$
\operatorname{pbw}^{\nabla}:\left(\Gamma\left(S\left(T_{\mathcal{M}}\right)\right), \mathcal{L}_{Q}\right) \rightarrow\left(\mathcal{D}(\mathcal{M}), \mathcal{L}_{Q}^{\mathcal{D}}\right)
$$

is an isomorphism of $d g$ coalgebras over $\left(C^{\infty}(\mathcal{M}), Q\right)$.

The Atiyah class of a dg manifold was first introduced by Shoikhet [49] in terms of Lie algebra cohomology and 1-jets of tangent bundles, appeared also in the work of Lyakhovich-MosmanSharapov [34, Footnote 6], and was studied systematically in [38]. The Atiyah class of dg manifolds plays a crucial role in the Kontsevich-Duflo type theorem for dg manifolds [33, 51]. Below we recall its definition in terms of affine connections [38].

\footnotetext{
${ }^{1}$ See $[57]$.
} 
Let $(\mathcal{M}, Q)$ be a dg manifold. Given an affine connection $\nabla$ on $\mathcal{M}$, consider the $(1,2)$-tensor $\operatorname{At}_{(\mathcal{M}, Q)}^{\nabla} \in \Gamma\left(\mathcal{M} ; T_{\mathcal{M}}^{\vee} \otimes \operatorname{End}\left(T_{\mathcal{M}}\right)\right)$ of degree +1 defined by the relation

$$
\operatorname{At}_{(\mathcal{M}, Q)}^{\nabla}(X, Y)=\left[Q, \nabla_{X} Y\right]-\nabla_{[Q, X]} Y-(-1)^{|X|} \nabla_{X}[Q, Y]
$$

for any homogeneous vector fields $X, Y \in \mathfrak{X}(\mathcal{M})$. Since $\mathcal{L}_{Q}\left(\operatorname{At}_{(\mathcal{M}, Q)}^{\nabla}\right)=0$, the element $\operatorname{At}_{(\mathcal{M}, Q)}^{\nabla}$ is a 1-cocycle called the Atiyah cocycle associated with the affine connection $\nabla$. The cohomology class

$$
\alpha_{(\mathcal{M}, Q)}:=\left[\operatorname{At}_{(\mathcal{M}, Q)}^{\nabla}\right] \in H^{1}\left(\Gamma\left(\mathcal{M} ; T_{\mathcal{M}}^{\vee} \otimes \operatorname{End}\left(T_{\mathcal{M}}\right)\right)^{\bullet}, \mathcal{Q}\right)
$$

does not depend on the choice of connection $\nabla$, and therefore is an intrinsic characteristic class called Atiyah class of the dg manifold $(\mathcal{M}, Q)[38]$ — see Proposition 2.5.

As shown by the pioneering work of Kapranov [25, 42, 43], the Atiyah class of a holomorphic vector bundle gives rise to $L_{\infty}[1]$ algebras. These $L_{\infty}$ [1] algebras play an important role in derived geometry [10, 36, 42] and the construction of Rozansky-Witten invariants [25, 27, 43, 45, 53].

It is natural to expect that the Atiyah cocycle of a dg manifold gives rise to an $L_{\infty}$ [1] algebra in a similar fashion. This is indeed true: the following theorem was announced in [38], but a proof was omitted. We will give a complete proof in the present paper.

Theorem B (Theorem 4.4). Let $(\mathcal{M}, Q)$ be a dg manifold. Each choice of an affine connection $\nabla$ on $\mathcal{M}$ determines an $L_{\infty}[1]$ algebra structure on the space of vector fields $\mathfrak{X}(\mathcal{M})$. While the unary bracket $\lambda_{1}: S^{1}(\mathfrak{X}(\mathcal{M})) \rightarrow \mathfrak{X}(\mathcal{M})$ is the Lie derivative $\mathcal{L}_{Q}$ along the homological vector field, the higher multibrackets $\lambda_{k}: S^{k}(\mathfrak{X}(\mathcal{M})) \rightarrow \mathfrak{X}(\mathcal{M})$, with $k \geq 2$, arise as the composition

$$
\lambda_{k}: S^{k}(\mathfrak{X}(\mathcal{M})) \rightarrow \Gamma\left(S^{k}\left(T_{\mathcal{M}}\right)\right) \stackrel{R_{k}}{\longrightarrow} \mathfrak{X}(\mathcal{M})
$$

induced by a family of sections $\left\{R_{k}\right\}_{k \geq 2}$ of the vector bundles $S^{k}\left(T_{\mathcal{M}}^{\vee}\right) \otimes T_{\mathcal{M}}$ starting with $R_{2}=-\operatorname{At}_{(\mathcal{M}, Q)}^{\nabla}$.

Furthermore, the $L_{\infty}[1]$ algebra structures on $\mathfrak{X}(\mathcal{M})$ arising from different choices of connections are all canonically isomorphic.

The $L_{\infty}$ [1] algebras arising in this way are called the Kapranov $L_{\infty}[1]$ algebras of the dg manifold. Our proof of Theorem B is very much inspired by Kapranov's construction [25, Theorem 2.8.2]. Essentially, we endow $\Gamma\left(S\left(T_{\mathcal{M}}\right)\right)$ with a dg coalgebra structure over $\left(C^{\infty}(\mathcal{M}), Q\right)$ using the PBW map (2) and the dg coalgebra $\left(\mathcal{D}(\mathcal{M}), \mathcal{L}_{Q}^{\mathcal{D}}\right)$, whose dual dg algebra can be considered as a kind of "the algebra of functions" on the "formal neighborhood" of the diagonal $\Delta$ of the product dg manifold $(\mathcal{M} \times \mathcal{M},(Q, Q))$. By construction, $\mathrm{pbw}^{\nabla}$ is a formal exponential map identifying a 'formal neighborhood' of the zero section of $T_{\mathcal{M}}$ to a 'formal neighborhood' of the diagonal $\Delta$ of the product manifold $\mathcal{M} \times \mathcal{M}$. The dg coalgebra structure on $\mathcal{D}(\mathcal{M})$ associated with the homological vector field $(Q, Q)$ on $\mathcal{M} \times \mathcal{M}$ can be pulled back through this formal exponential map so as to obtain a dg coalgebra $\left(S(\mathfrak{X}(M)), \delta^{\nabla}\right)$, which in turn induces an $L_{\infty}[1]$ algebra on $\mathfrak{X}(\mathcal{M})$.

The Kapranov $L_{\infty}[1]$ algebra of a dg manifold as in Theorem B is completely determined by the Atiyah 1-cocycle and the sections

$$
R_{k} \in \Gamma\left(S^{k}\left(T_{\mathcal{M}}^{\vee}\right) \otimes T_{\mathcal{M}}\right) \cong \Gamma\left(\operatorname{Hom}\left(S^{k}\left(T_{\mathcal{M}}\right), T_{\mathcal{M}}\right)\right)
$$

for $k \geq 3$. It is thus natural to wonder whether the $R_{k}$ 's can be described explicitly.

For the $L_{\infty}[1]$ algebra structure on the Dolbeault complex $\left(\Omega^{0, \bullet}\left(T_{X}^{1,0}\right), \bar{\partial}\right)$ associated with the Atiyah class of the holomorphic tangent bundle $T_{X}$ of a Kähler manifold $X$, Kapranov showed that the multibrackets can be described explicitly by a very simple formula [25]: Equation (4) below. Consider the $\mathbb{C}$-linear extension of the Levi-Civita connection of the Kähler manifold $X$; this is a $T_{X}^{\mathbb{C}}$-connection $\nabla$ on $T_{X}^{\mathbb{C}}$. Since $X$ is Kähler, $\nabla$ induces a $T_{X}^{\mathbb{C}}$-connection on $T_{X}^{1,0}$, also denoted by $\nabla$, 
which decomposes as the sum $\nabla=\nabla^{\bar{\partial}}+\nabla^{1,0}$ of the canonical flat $T_{X}^{0,1}$-connection $\nabla^{\bar{\partial}}$ on $T_{X}^{1,0}$ and some $T_{X}^{1,0}$-connection $\nabla^{1,0}$ on $T_{X}^{1,0}$. Since $\nabla^{1,0}$ is torsion-free and $d^{\nabla^{1,0}} \circ d^{\nabla^{1,0}}=0 \in \Omega^{2,0}\left(\right.$ End $\left.T^{1,0}\right)$, the curvature of $\nabla$ is $R^{\nabla}=\left[d^{\nabla^{\bar{\partial}}}, d^{\nabla^{1,0}}\right]$, which equals to $R_{2} \in \Omega^{0,1}\left(S^{2}\left(T_{X}^{1,0}\right)^{\vee} \otimes T_{X}^{1,0}\right)$, the Dolbeault representative of the Atiyah 1-cocycle of the holomorphic tangent bundle $T_{X}$. Kapranov proved [25, Theorem 2.6] that, for $k \geqslant 2$, the $k$-th multibracket $\lambda_{k}$ on the Dolbeault complex $\left(\Omega^{0, \bullet}\left(T_{X}^{1,0}\right), \bar{\partial}\right)$ is the composition of the wedge product

$$
\Omega^{0, j_{1}}\left(T_{X}^{1,0}\right) \otimes \cdots \otimes \Omega^{0, j_{k}}\left(T_{X}^{1,0}\right) \rightarrow \Omega^{0, j_{1}+\cdots+j_{k}}\left(\left(T_{X}^{1,0}\right)^{\otimes k}\right)
$$

with the map

$$
\Omega^{0, j_{1}+\cdots+j_{k}}\left(\left(T_{X}^{1,0}\right)^{\otimes k}\right) \rightarrow \Omega^{0, j_{1}+\cdots+j_{k}+1}\left(T_{X}^{1,0}\right)
$$

induced by

and that, for $k \geqslant 3$,

$$
R_{k} \in \Omega^{0,1}\left(S^{k}\left(T_{X}^{1,0}\right)^{\vee} \otimes T_{X}^{1,0}\right) \subset \Omega^{0,1}\left(\operatorname{Hom}\left(\left(T_{X}^{1,0}\right)^{\otimes k}, T_{X}^{1,0}\right)\right)
$$

$$
R_{k}=d^{\nabla^{1,0}} R_{k-1} \quad \in \Omega^{0,1}\left(S^{k}\left(T_{X}^{1,0}\right)^{\vee} \otimes T_{X}^{1,0}\right) .
$$

If $X$ is a mere complex manifold rather than a Kähler manifold, the relation between the $R_{k}$ 's is more complicated: it involves the Atiyah 1-cocycle $R_{2}$, the curvature of $\nabla^{1,0}$, and their higher covariant derivatives. Nevertheless, recursive computations are still possible as shown in [31].

In the present paper, we prove that a similar characterization of the higher multibrackets holds for the Kapranov $L_{\infty}[1]$ algebra of a $d g$ manifold:

Theorem C (Theorem 4.7).

(1) The sections $R_{n} \in \Gamma\left(S^{n}\left(T_{\mathcal{M}}^{\vee}\right) \otimes T_{\mathcal{M}}\right)$, with $n \geq 3$, are completely determined, by way of a recursive formula, by the Atiyah cocycle $\operatorname{At}_{(\mathcal{M}, Q)}^{\nabla}$, the curvature $R^{\nabla}$, and their higher covariant derivatives - see (40).

(2) In particular, if $R^{\nabla}=0$, then $R_{2}=-\mathrm{At}_{(\mathcal{M}, Q)}^{\nabla}$ and $R_{n}=\frac{1}{n} \widetilde{d^{\nabla}} R_{n-1}$, for all $n \geq 3$.

Finally, we investigate the Kapranov $L_{\infty}$ [1] algebras arising from two classes of examples of dg manifolds: those corresponding to finite dimensional $L_{\infty}$ [1] algebras as described in Example 2.2, and those corresponding to manifolds endowed with integrable distributions, which include not only foliated manifolds but also complex manifolds as described in Example 2.3. For the dg manifold $\left(\mathfrak{g}[1], d_{\mathrm{CE}}\right)$ associated with a finite-dimensional $L_{\infty}[1]$ algebra $\mathfrak{g}[1]$, we prove that the multibrackets of the Kapranov $L_{\infty}[1]$ algebra structure on $\mathfrak{X}(\mathfrak{g}[1]) \cong \operatorname{Hom}(S(\mathfrak{g}[1]), \mathfrak{g}[1])$ can be expressed in terms of the multibrackets of the $L_{\infty}$ [1] algebra $\mathfrak{g}[1]$ — see Proposition 5.8. We also compute the Atiyah class of the dg manifold $\left(\mathfrak{g}[1], d_{\mathrm{CE}}\right)$ in terms of Chevalley-Eilenberg cohomology of $\mathfrak{g}[1]$ with values in the tensor product of adjoint and coadjoint modules $(\mathfrak{g}[1])^{\vee} \otimes(\mathfrak{g}[1])^{\vee} \otimes \mathfrak{g}[1]$ - see Proposition 5.6. For the dg manifold $\left(F[1], d_{F}\right)$ arising from an integrable distribution $F \subseteq T_{\mathbb{K}} M$ on a smooth manifold $M$, we show that the Kapranov $L_{\infty}$ [1] algebra structure on $\mathfrak{X}(F[1])$ is quasi-isomorphic to the $L_{\infty}$ [1] algebra $\Omega_{F}^{\bullet}\left(T_{\mathbb{K}} M / F\right)$ arising from the Lie pair $\left(T_{\mathbb{K}} M, F\right)$, which was studied extensively in [30, 31, 15]. In particular, for the $\mathrm{dg}$ manifold $\left(T_{X}^{0,1}[1], \bar{\partial}\right)$ associated with a complex manifold $X$, the Kapranov $L_{\infty}$ [1] algebra structure on $\mathfrak{X}\left(T_{X}^{0,1}[1]\right)$ is quasi-isomorphic to the $L_{\infty}$ [1] algebra structure on the Dolbeault complex $\left(\Omega^{0, \bullet}\left(T_{X}^{1,0}\right), \bar{\partial}\right)$ associated with the Atiyah class of the holomorphic tangent bundle $T_{X}$. Moreover, each map $\phi_{k}$ in the quasi-isomorphism $\left\{\phi_{k}\right\}_{k \geq 1}$ is $\Omega_{X}^{0, \bullet}$-multilinear - see Corollary 5.15.

Note that Bandiera $[2,3]$ proved that, when $X$ is a Kähler manifold, the Kapranov $L_{\infty}[1]$ algebra structure on $\Omega^{0, \bullet}\left(T_{X}^{1,0}\right)$ is homotopy abelian over the field $\mathbb{C}$. It would be interesting to investigate if the $L_{\infty}[1]$ algebra structure of Theorem $\mathrm{B}$ on the space $\mathfrak{X}(\mathcal{M})$ of vector fields over a dg manifold 
$(\mathcal{M}, Q)$ is homotopy abelian over the field $\mathbb{K}$, possibly by extending the techniques developed in $[2$, $3]$.

Notations and conventions. Throughout this paper, the symbol $\mathbb{K}$ denotes a field either $\mathbb{R}$ or $\mathbb{C}$.

We reserve the symbol $M$ to denote a smooth manifold (over $\mathbb{K}$ ) exclusively. The sheaf of smooth $\mathbb{K}$-valued functions on $M$ is denoted $\mathcal{O}_{M}=\mathcal{O}_{M}^{\mathbb{K}}$. The algebra of globally defined smooth functions on $M$ is $C^{\infty}(M)=\mathcal{O}_{M}(M)$.

A $(p, q)$-shuffle is a permutation $\sigma$ of the set $\{1,2, \cdots, p+q\}$ such that $\sigma(1)<\cdots<\sigma(p)$ and $\sigma(p+1)<\cdots<\sigma(p+q)$. The set of $(p, q)$-shuffles will be denoted by $\mathfrak{S}_{p}^{q}$.

We use Sweedler's (sumless) notation for the comultiplication $\Delta$ in any coalgebra $C$ :

$$
\Delta(c)=\sum_{(c)} c_{(1)} \otimes c_{(2)}=c_{(1)} \otimes c_{(2)}, \quad \forall c \in C .
$$

All gradings in this paper are $\mathbb{Z}$-gradings and $\mathcal{M}$ will always be a finite dimensional graded manifold. Throughout the paper, 'dg' means 'differential graded.'

Given a graded vector space $V$, the suspension of $V$ is denoted by $V[1]$ satisfying $V[1]^{n}=V^{n+1}$. We denote the (internal) degree of an element $v \in V$ by $|v|$.

Many equations throughout the paper have the following general shape:

$$
A\left(X_{1}, X_{2}, \ldots, X_{n}\right)=(-1)^{\sum_{(i, j) \in \mathscr{K}}\left|X_{\sigma(i)}\right|\left|X_{\sigma(j)}\right|} B\left(X_{\sigma(1)}, X_{\sigma(2)}, \ldots, X_{\sigma(n)}\right),
$$

where $X_{1}, X_{2}, \ldots, X_{n}$ is a finite collection of $\mathbb{Z}$-graded objects; $\sigma$ is a permutation of the set of indices $\{1,2, \ldots, n\} ; \mathscr{K}$ is the set of couples $(i, j)$ of elements of $\{1,2 \ldots, n\}$ such that $i<j$ and $\sigma(i)>\sigma(j)$; and $A$ and $B$ are $n$-ary operations on the $\mathbb{Z}$-graded objects $X_{1}, X_{2}, \ldots, X_{n}$ whose

output is an object of degree $\left|X_{1}\right|+\left|X_{2}\right|+\cdots+\left|X_{n}\right|$. The factor $(-1)^{\sum_{(i, j) \in \mathscr{K}}\left|X_{\sigma(i)}\right|\left|X_{\sigma(j)}\right|}$ appearing in the right hand side of (5) is called the Koszul sign of the permutation $\sigma$ of the graded objects $X_{1}, X_{2}, \ldots, X_{n}$. It will customarily be abbreviated as $\varepsilon$ since its actual value - either +1 or -1 can be recovered from a careful inspection of both sides of the equation. We will also use the more explicit abbreviation $\varepsilon\left(X_{1}, X_{2}, \cdots, X_{n}\right)$ if the collection of $\mathbb{Z}$-graded objects begin permuted is not immediately clear. As explained by Boardman in [6], this sign is mostly inconsequential and it is not necessary to devote much attention or thought to it. In fact, the right hand side of (5) can be a sum of several terms so it would be more correct to say that the general shape of the equations is

$$
A\left(X_{1}, X_{2}, \ldots, X_{n}\right)=\sum_{k}(-1)^{\sum_{(i, j) \in \mathscr{K}_{k}}\left|X_{\sigma_{k}(i)}\right|\left|X_{\sigma_{k}(j)}\right|} B_{k}\left(X_{\sigma_{k}(1)}, X_{\sigma_{k}(2)}, \ldots, X_{\sigma_{k}(n)}\right) .
$$

\section{PRELiminaries}

2.1. dg manifolds. Let $M$ be a smooth manifold over $\mathbb{K}$, and $\mathcal{O}_{M}$ be the sheaf of $\mathbb{K}$-valued smooth functions over $M$. A graded manifold $\mathcal{M}$ with support $M$ consists of a sheaf $\mathcal{A}$ of graded commutative $\mathcal{O}_{M}$-algebra on $M$ such that there is a $\mathbb{Z}$-graded vector space $V$ satisfying

$$
\mathcal{A}(U) \cong \mathcal{O}_{M}(U) \otimes_{\mathbb{K}} \operatorname{Hom}_{\mathbb{K}}(S(V), \mathbb{K}) \cong \mathcal{O}_{M}(U) \otimes_{\mathbb{K}} \widehat{S}\left(V^{\vee}\right)
$$

for sufficiently small open set $U \subset M$. The global section of the sheaf $\mathcal{A}$ will be denoted by $C^{\infty}(\mathcal{M})=\mathcal{A}(M)$. We say a graded manifold $\mathcal{M}$ is finite dimensional if $\operatorname{dim} M<\infty$ and $\operatorname{dim} V<\infty$. Throughout this paper, graded manifold $\mathcal{M}$ will always be finite dimensional.

Remark 2.1. In the literature, the sheaf of functions $\mathcal{A}$ over a graded manifold is defined by $\mathcal{A}(U)=\mathcal{O}_{M}(U) \otimes_{\mathbb{K}} S\left(V^{\vee}\right)$ for sufficiently small open subsets $U$ of $M$. Here, however, we allow for formal power series rather than polynomials: $\mathcal{A}(U)=\mathcal{O}_{M}(U) \otimes_{\mathbb{K}} \widehat{S}\left(V^{\vee}\right)$ for sufficiently small 
open subsets $U$ of $M$. Consequently, when we write 'dg manifold' $(\mathcal{M}, Q)$, we actually mean formal dg manifold in Kontsevich's sense [26, Section 4.1].

By $\mathcal{I}_{\mathcal{A}}$, we denote the sheaf of ideal of $\mathcal{A}$ consisting of functions vanishing at the support $M$ of $\mathcal{M}$. That is, for sufficiently small $U \subset M$,

$$
\mathcal{I}_{\mathcal{A}}(U) \cong \mathcal{O}_{M}(U) \otimes_{\mathbb{K}} \widehat{S}^{\geq 1}\left(V^{\vee}\right) .
$$

Given graded manifolds $\mathcal{M}=(M, \mathcal{A})$ and $\mathcal{N}=(N, \mathcal{B})$, a morphism $\mathcal{M} \rightarrow \mathcal{N}$ of graded manifolds consists of a pair $(f, \psi)$, where $f: M \rightarrow N$ is a morphism of smooth manifolds and $\psi: f^{*} \mathcal{B} \rightarrow \mathcal{A}$ is a morphism of sheaves of graded commutative $\mathcal{O}_{M}$-algebras such that $\psi\left(f^{*} \mathcal{I}_{\mathcal{B}}\right) \subset \mathcal{I}_{\mathcal{A}}$. We often use the notation $\phi: \mathcal{M} \rightarrow \mathcal{N}$ to denote such a morphism. Then $\psi=\phi^{*}$. Also, we write $\phi^{*}: C^{\infty}(\mathcal{N}) \rightarrow C^{\infty}(\mathcal{M})$ to denote the morphism on global sections. Note that the condition $\psi\left(f^{*} \mathcal{I}_{\mathcal{B}}\right) \subset \mathcal{I}_{\mathcal{A}}$ is equivalent to $\psi$ being continuous w.r.t the $\mathcal{I}$-adic topology.

Vector bundles in the category of graded manifolds are called graded vector bundles. Given a graded vector bundle $\Phi: \mathcal{E} \rightarrow \mathcal{M}$, a section $s: \mathcal{M} \rightarrow \mathcal{E}$ of $\mathcal{E}$ over $\mathcal{M}$ is a morphism of graded manifolds such that $\Phi \circ s=\mathrm{id}_{\mathcal{M}}$. We write the $C^{\infty}(\mathcal{M})$-module of sections of $\mathcal{E}$ over $\mathcal{M}$ by the usual notation $\Gamma(\mathcal{E})=\Gamma(\mathcal{M} ; \mathcal{E})$.

For a graded manifold $\mathcal{M}$ with support $M$, its tangent bundle $T_{\mathcal{M}}$ is a graded manifold with support $T_{M}$ and is a graded vector bundle over $\mathcal{M}$. Its sections are called vector fields on $\mathcal{M}$ and the space of vector fields $\Gamma\left(\mathcal{M} ; T_{\mathcal{M}}\right)=\Gamma\left(T_{\mathcal{M}}\right)$ can be identified with that of graded derivations of $C^{\infty}(\mathcal{M})$. We also write $\Gamma\left(\mathcal{M} ; T_{\mathcal{M}}\right)=\mathfrak{X}(\mathcal{M})$. Observe that $\mathfrak{X}(\mathcal{M})$ admits a Lie algebra structure, whose Lie bracket coincides with the graded commutator

$$
[X, Y]=X \circ Y-(-1)^{|X| \cdot|Y|} Y \circ X
$$

for homogeneous elements $X, Y \in \mathfrak{X}(\mathcal{M})$ regarded as derivations of $C^{\infty}(\mathcal{M})$. Indeed $T_{\mathcal{M}}$ is a graded Lie algebroid [37].

A differential graded manifold (dg manifold in short) is a graded manifold $\mathcal{M}$ together with a homological vector field, i.e. a vector field $Q \in \mathfrak{X}(\mathcal{M})$ of degree +1 satisfying $[Q, Q]=Q \circ Q+Q \circ Q=0$. For a dg manifold $(\mathcal{M}, Q)$, its tangent bundle $T_{\mathcal{M}}$ is naturally a dg manifold, with the homological vector field being the complete $\operatorname{lift}^{2}$ of $Q$, and in fact $T_{\mathcal{M}}$ is a dg Lie algebroid over $\mathcal{M}[37,38]$.

Example 2.2. Let $\mathfrak{g}$ be a finite dimensional Lie algebra. Then $\left(\mathfrak{g}[1], d_{\mathrm{CE}}\right)$ is a $\mathrm{dg}$ manifold - its algebra of functions is $C^{\infty}(\mathfrak{g}[1]) \cong \Lambda^{\bullet} \mathfrak{g}^{\vee}$ and its homological vector field $Q$ is the Chevalley-Eilenberg differential $d_{\mathrm{CE}}$.

This construction admits an 'up to homotopy' version: Given a $\mathbb{Z}$-graded finite dimensional vector space $\mathfrak{g}=\bigoplus_{i \in \mathbb{Z}} \mathfrak{g}_{i}$, the graded manifold $\mathfrak{g}[1]$ is a dg manifold, i.e. admits a homological vector field, if and only if $\mathfrak{g}$ admits a structure of curved $L_{\infty}$ algebra.

Example 2.3. Let $M$ be a smooth manifold. Then $\left(T_{M}[1], d_{\mathrm{dR}}\right)$ is a dg manifold - its algebra of functions is $C^{\infty}\left(T_{M}[1]\right) \cong \Omega^{\bullet}(M)$ and its homological vector field $Q$ is the de Rham differential $d_{\mathrm{dR}}$. Likewise, a complex manifold $X$ gives rise to a dg manifold $\left(T_{X}^{0,1}[1], \bar{\partial}\right)$ whose algebra of functions $C^{\infty}\left(T_{X}^{0,1}[1]\right)$ is $\Omega^{0, \bullet}(X)$ and whose homological vector field $Q$ is the Dolbeault operator $\bar{\partial}$.

Example 2.4. Let $s$ be a smooth section of a vector bundle $E \rightarrow M$. Then $\left(E[-1], \iota_{s}\right)$ is a dg manifold - its algebra of functions is $C^{\infty}(E[-1]) \cong \Gamma\left(\Lambda^{-\bullet} E^{\vee}\right)$ and its homological vector field is $Q=\iota_{s}$, the interior product with $s$. This dg manifold can be thought of as a smooth model for the (possibly singular) intersection of $s$ with the zero section of the vector bundle $E$, and is often called a 'derived intersection', or a quasi-smooth derived manifold [5].

${ }^{2}$ It is also called tangent lift in the literature [38, 33]. 
Both situations in Example 2.3 are special instances of Lie algebroids, while Example 2.4 is a special case of derived manifolds [5].

2.2. Atiyah class. Let $\mathcal{M}$ be a graded manifold and $\mathcal{E}$ be a graded vector bundle over $\mathcal{M}$. We say a $\mathbb{K}$-linear map

$$
\nabla: \mathfrak{X}(\mathcal{M}) \otimes_{\mathbb{K}} \Gamma(\mathcal{E}) \rightarrow \Gamma(\mathcal{E})
$$

of degree 0 is a linear connection on $\mathcal{E}$ over $\mathcal{M}$ if the following axioms are satisfied:

(1) $C^{\infty}(\mathcal{M})$-linear in the first argument: $\nabla_{f X} s=f \nabla_{X} s$.

(2) $\nabla_{X}$ is a derivative in the second argument: $\nabla_{X}(f s)=X(f) s+(-1)^{|f| \cdot|X|} f \nabla_{X} s$,

where $f \in C^{\infty}(\mathcal{M})$ and $X \in \mathfrak{X}(\mathcal{M})$ are homogeneous elements, and $s \in \Gamma(\mathcal{E})$.

The covariant derivative associated to a linear connection $\nabla$ is the $\mathbb{K}$-linear map

$$
d^{\nabla}: \Gamma\left(\Lambda^{p} T_{\mathcal{M}}^{\vee} \otimes \mathcal{E}\right) \rightarrow \Gamma\left(\Lambda^{p+1} T_{\mathcal{M}}^{\vee} \otimes \mathcal{E}\right)
$$

of (internal) degree 0 , defined by

$$
\begin{aligned}
\left(d^{\nabla} \omega\right)\left(X_{1} \wedge \cdots \wedge X_{p+1}\right)= & \sum_{i=1}^{p+1}(-1)^{i+1} \varepsilon \cdot \nabla_{X_{i}}\left(\omega\left(X_{1} \wedge \cdots \wedge \widehat{X}_{i} \wedge \cdots \wedge X_{p+1}\right)\right) \\
& +\sum_{i<j}(-1)^{i+j} \varepsilon \cdot \omega\left(\left[X_{i}, X_{j}\right] \wedge X_{1} \wedge \cdots \wedge \widehat{X}_{i} \wedge \cdots \wedge \widehat{X}_{j} \wedge \cdots \wedge X_{p+1}\right),
\end{aligned}
$$

for all homogeneous $\omega \in \Gamma\left(\Lambda^{p} T_{\mathcal{M}}^{\vee} \otimes \mathcal{E}\right)$ and $X_{1}, \cdots, X_{p+1} \in \mathfrak{X}(\mathcal{M})$. The symbol $\varepsilon=\varepsilon\left(\omega, X_{1}, \cdots, X_{p+1}\right)$ denotes the Koszul signs arising from the reordering of the homogeneous objects $\omega, X_{1}, \cdots, X_{p+1}$ in each term of the right hand side.

We say $\nabla$ is an affine connection on $\mathcal{M}$ if it is a linear connection on $T_{\mathcal{M}}$ over $\mathcal{M}$. Given an affine connection $\nabla$ on $\mathcal{M}$, the $(1,2)$-tensor $T^{\nabla} \in \Gamma\left(T_{\mathcal{M}}^{\vee} \otimes T_{\mathcal{M}}^{\vee} \otimes T_{\mathcal{M}}\right)$ of degree 0 , defined by

$$
T^{\nabla}(X, Y)=\nabla_{X} Y-(-1)^{|X| \cdot|Y|} \nabla_{Y} X-[X, Y]
$$

for any homogeneous vector fields $X, Y \in \mathfrak{X}(\mathcal{M})$, is called the torsion of $\nabla$. We say an affine connection $\nabla$ is torsion-free if $T^{\nabla}=0$. It is well known that affine torsion-free connections always exist [32].

The curvature of an affine connection $\nabla$ is the $(1,3)$-tensor $R^{\nabla} \in \Omega^{2}\left(\mathcal{M}\right.$, $\left.\operatorname{End}\left(T_{\mathcal{M}}\right)\right)$ of degree 0 , defined by

$$
R^{\nabla}(X, Y) Z=\nabla_{X} \nabla_{Y} Z-(-1)^{|X| \cdot|Y|} \nabla_{Y} \nabla_{X} Z-\nabla_{[X, Y]} Z
$$

for any homogeneous vector fields $X, Y, Z \in \mathfrak{X}(\mathcal{M})$. If the curvature $R^{\nabla}$ vanishes identically, the affine connection $\nabla$ is called flat.

Let $(\mathcal{M}, Q)$ be a dg manifold. We define an operator $\mathcal{Q}$ of degree +1 on the graded $C^{\infty}(\mathcal{M})$-module $\Gamma\left(\mathcal{M} ; T_{\mathcal{M}}^{\vee} \otimes \operatorname{End}\left(T_{\mathcal{M}}\right)\right):$

$$
\mathcal{Q}: \Gamma\left(\mathcal{M} ; T_{\mathcal{M}}^{\vee} \otimes \operatorname{End}\left(T_{\mathcal{M}}\right)\right)^{\bullet} \rightarrow \Gamma\left(\mathcal{M} ; T_{\mathcal{M}}^{\vee} \otimes \operatorname{End}\left(T_{\mathcal{M}}\right)\right)^{\bullet+1}
$$

by the Lie derivative along the homological vector field $Q$ :

$$
(\mathcal{Q} F)(X, Y)=[Q, F(X, Y)]-(-1)^{k} F([Q, X], Y)-(-1)^{k+|X|} F(X,[Q, Y])
$$

for any section $F \in \Gamma\left(\mathcal{M} ; T_{\mathcal{M}}^{\vee} \otimes \operatorname{End}\left(T_{\mathcal{M}}\right)\right)^{k}$ of degree $k$ and homogeneous vector fields $X, Y \in \mathfrak{X}(\mathcal{M})$. One can easily check that $\mathcal{Q}^{2}=0$. Therefore

$$
\left(\Gamma\left(\mathcal{M} ; T_{\mathcal{M}}^{\vee} \otimes \operatorname{End}\left(T_{\mathcal{M}}\right)\right)^{\bullet}, \mathcal{Q}\right)
$$

is a cochain complex. 
Now given an affine connection $\nabla$, consider the $(1,2)$-tensor $\operatorname{At}_{(\mathcal{M}, Q)}^{\nabla} \in \Gamma\left(\mathcal{M} ; T_{\mathcal{M}}^{\vee} \otimes \operatorname{End}\left(T_{\mathcal{M}}\right)\right)$ of degree +1 , defined by

$$
\operatorname{At}_{(\mathcal{M}, Q)}^{\nabla}(X, Y)=\left[Q, \nabla_{X} Y\right]-\nabla_{[Q, X]} Y-(-1)^{|X|} \nabla_{X}[Q, Y]
$$

for any homogeneous vector fields $X, Y \in \mathfrak{X}(\mathcal{M})$.

Proposition 2.5 ([38]). In the above setting, the following statements hold.

(1) If the affine connection $\nabla$ on $\mathcal{M}$ is torsion-free, then $\operatorname{At}_{(\mathcal{M}, Q)}^{\nabla} \in \Gamma\left(\mathcal{M} ; S^{2}\left(T_{\mathcal{M}}^{\vee}\right) \otimes T_{\mathcal{M}}\right)$. In other words,

$$
\operatorname{At}_{(\mathcal{M}, Q)}^{\nabla}(X, Y)=(-1)^{|X| \cdot|Y|} \operatorname{At}_{(\mathcal{M}, Q)}^{\nabla}(Y, X) .
$$

(2) The element $\operatorname{At}_{(\mathcal{M}, Q)}^{\nabla} \in \Gamma\left(\mathcal{M} ; T_{\mathcal{M}}^{\vee} \otimes \operatorname{End}\left(T_{\mathcal{M}}\right)\right)^{1}$ is a 1-cocycle.

(3) The cohomology class $\left[\operatorname{At}_{(\mathcal{M}, Q)}^{\nabla}\right]$ does not depend on the choice of connection.

The element $\operatorname{At}_{(\mathcal{M}, Q)}^{\nabla}$ is called the Atiyah cocycle associated with the affine connection $\nabla$. The cohomology class $\alpha_{(\mathcal{M}, Q)}:=\left[\operatorname{At}_{(\mathcal{M}, Q)}^{\nabla}\right] \in H^{1}\left(\Gamma\left(\mathcal{M} ; T_{\mathcal{M}}^{\vee} \otimes \operatorname{End}\left(T_{\mathcal{M}}\right)\right)^{\bullet}, \mathcal{Q}\right)$ is called the Atiyah class of the $\operatorname{dg}$ manifold $(\mathcal{M}, Q)[38]$. See also [49] and [34, Footnote 6].

\section{Formal exponential MAP OF DG MANifoldS}

\section{1. dg coalgebras.}

3.1.1. $d g$ coalgebras. Let $\mathcal{R}$ be a graded commutative ring. A graded coalgebra $C$ over $\mathcal{R}$ is a graded $\mathcal{R}$-module equipped with an $\mathcal{R}$-linear map $\Delta: C \rightarrow C \otimes_{\mathcal{R}} C$ of degree 0 called comultiplication satisfying the following conditions:

(1) (Coassociativity)

$$
\left(\Delta \otimes \operatorname{id}_{C}\right) \circ \Delta=\left(\operatorname{id}_{C} \otimes \Delta\right) \circ \Delta: C \rightarrow C \otimes_{\mathcal{R}} C \otimes_{\mathcal{R}} C .
$$

(2) (Counit) There is an $\mathcal{R}$-linear map $\epsilon: C \rightarrow \mathcal{R}$ of degree 0 such that

$$
(\epsilon \otimes \mathrm{id}) \circ \Delta=(\mathrm{id} \otimes \epsilon) \circ \Delta=\mathrm{id}_{C} .
$$

Let tw :C $\otimes_{\mathcal{R}} C \rightarrow C \otimes_{\mathcal{R}} C$ be the map defined by

$$
\operatorname{tw}\left(c_{1} \otimes c_{2}\right)=(-1)^{\left|c_{1}\right| \cdot\left|c_{2}\right|} c_{2} \otimes c_{1},
$$

for homogeneous elements $c_{1}, c_{2} \in C$. A graded coalgebra $C$ is called cocommutative if it satisfies $\Delta=\operatorname{tw} \circ \Delta$.

An $\mathcal{R}$-linear map $\phi: C \rightarrow C$ satisfying

$$
\Delta \circ \phi=\left(\operatorname{id}_{C} \otimes \phi+\phi \otimes \operatorname{id}_{C}\right) \circ \Delta
$$

is called an $\mathcal{R}$-coderivation of the graded $\mathcal{R}$-coalgebra $C$. We denote the collection of all $\mathcal{R}$ coderivations of $C$ by $\operatorname{coDer}_{\mathcal{R}}(C)$.

Let $\left(\mathcal{R}, d_{\mathcal{R}}\right)$ be a $\operatorname{dg}$ commutative ring, and $\left(C, d_{C}\right)$ be a $\operatorname{dg}\left(\mathcal{R}, d_{\mathcal{R}}\right)$-module. Then the map

$$
d_{C \otimes 2}: C \otimes_{\mathcal{R}} C \rightarrow C \otimes_{\mathcal{R}} C
$$

defined by

$$
d_{C \otimes 2}\left(c_{1} \otimes c_{2}\right)=d_{C}\left(c_{1}\right) \otimes c_{2}+(-1)^{\left|c_{1}\right|} c_{1} \otimes d_{C}\left(c_{2}\right)
$$

for homogeneous elements $c_{1}, c_{2} \in C$, is a well-defined degree +1 differential. Such a differential is called the induced differential on $C \otimes_{\mathcal{R}} C$. 
Definition 3.1. Let $\left(\mathcal{R}, d_{\mathcal{R}}\right)$ be a dg commutative ring. A $\boldsymbol{d g}$ coalgebra $\left(C, d_{C}\right)$ over $\left(\mathcal{R}, d_{\mathcal{R}}\right)$ is a $\operatorname{dg}\left(\mathcal{R}, d_{\mathcal{R}}\right)$-module $\left(C, d_{C}\right)$, equipped with a graded coalgebra structure on $C$ over $\mathcal{R}$ where the comultiplication and the counit map respect the differentials. That is,

$$
\begin{gathered}
\Delta \circ d_{C}=d_{C \otimes 2} \circ \Delta, \\
\epsilon \circ d_{C}=d_{\mathcal{R}} \circ \epsilon
\end{gathered}
$$

where $\Delta: C \rightarrow C \otimes_{\mathcal{R}} C$ is the comultiplication and $\epsilon: C \rightarrow \mathcal{R}$ is the counit map.

3.1.2. dg coalgebras associated to $d g$ manifolds. Any $\operatorname{dg}$ manifold $(\mathcal{M}, Q)$ determines a pair of $\mathrm{dg}$ coalgebras over the dg ring $\left(C^{\infty}(\mathcal{M}), Q\right)$, namely $\mathcal{D}(\mathcal{M})$ and $\Gamma\left(S\left(T_{\mathcal{M}}\right)\right)$. Below we will briefly describe these dg coalgebra structures. In the sequel, unless specified otherwise, we will always identify $\left(\mathcal{R}, d_{\mathcal{R}}\right) \cong\left(C^{\infty}(\mathcal{M}), Q\right)$.

First, let us consider the dg coalgebra structure on the left $\mathcal{R}$-module $\mathcal{D}(\mathcal{M})$ of differential operators on $\mathcal{M}$.

The comultiplication

$$
\Delta: \mathcal{D}(\mathcal{M}) \rightarrow \mathcal{D}(\mathcal{M}) \otimes_{\mathcal{R}} \mathcal{D}(\mathcal{M})
$$

is defined by

$$
(\Delta D)(f \otimes g)=D(f \cdot g)
$$

where $f, g \in C^{\infty}(\mathcal{M})$ and $D \in \mathcal{D}(\mathcal{M})$.

The differential $\mathcal{L}_{Q}^{\mathcal{D}}: \mathcal{D}(\mathcal{M}) \rightarrow \mathcal{D}(\mathcal{M})$ is defined as the commutator with $Q$, which is also the Lie derivative along the homological vector field $Q$ :

$$
\mathcal{L}_{Q}^{\mathcal{D}}(D)=\llbracket Q, D \rrbracket=Q \cdot D-(-1)^{|D|} D \cdot Q
$$

for any $D \in \mathcal{D}(\mathcal{M})$, where $\llbracket-,-\rrbracket$ denotes the commutator on $\mathcal{D}(\mathcal{M})$.

The induced differential on $\mathcal{D}(\mathcal{M}) \otimes_{\mathcal{R}} \mathcal{D}(\mathcal{M})$ is again the Lie derivative $\mathcal{L}_{Q}^{\mathcal{D}}$, which coincides with $\llbracket Q,-\rrbracket$, with $\llbracket-,-\rrbracket$ being the Gerstenhaber bracket on polydifferential operators on $\mathcal{M}$.

The counit map

$$
\epsilon: \mathcal{D}(\mathcal{M}) \rightarrow C^{\infty}(\mathcal{M})
$$

is the canonical projection, which evaluates a differential operator $D$ on the constant function 1 .

Note that $\mathcal{D}(\mathcal{M})$ admits a natural ascending filtration by the order of differential operators

$$
C^{\infty}(\mathcal{M})=\mathcal{D}^{\leq 0}(\mathcal{M}) \subset \cdots \subset \mathcal{D}^{\leq n}(\mathcal{M}) \subset \cdots
$$

where $\mathcal{D}^{\leq n}(\mathcal{M})$ denotes the space of differential operators of order $\leq n$. The following proposition can be easily verified.

Proposition 3.2. For any $d g$ manifold $(\mathcal{M}, Q)$, the space of differential operators $\mathcal{D}(\mathcal{M})$ on $\mathcal{M}$, equipped with the comultiplication $\Delta$, the differential $\mathcal{L}_{Q}^{\mathcal{D}}$ and the counit $\epsilon$ as in (7), (8) and (9), is a filtered $d g$ cocommutative coalgebra over $\left(C^{\infty}(\mathcal{M}), Q\right)$.

Next we describe the dg coalgebra structure on the left $\mathcal{R}$-module $\Gamma\left(S\left(T_{\mathcal{M}}\right)\right)$.

The comultiplication

is given by

$$
\Delta: \Gamma\left(S\left(T_{\mathcal{M}}\right)\right) \rightarrow \Gamma\left(S\left(T_{\mathcal{M}}\right)\right) \otimes_{\mathcal{R}} \Gamma\left(S\left(T_{\mathcal{M}}\right)\right)
$$

$$
\begin{aligned}
\Delta\left(X_{1} \odot \cdots \odot X_{n}\right) & =1 \otimes\left(X_{1} \odot \cdots \odot X_{n}\right)+\left(X_{1} \odot \cdots \odot X_{n}\right) \otimes 1 \\
& +\sum_{k=1}^{n-1} \sum_{\sigma \in \mathfrak{S}_{k}^{n-k}} \varepsilon \cdot\left(X_{\sigma(1)} \odot \cdots \odot X_{\sigma(k)}\right) \otimes\left(X_{\sigma(k+1)} \odot \cdots \odot X_{\sigma(n)}\right),
\end{aligned}
$$


where $X_{1}, \cdots, X_{n} \in \Gamma\left(T_{\mathcal{M}}\right)$. The symbol $\mathfrak{S}_{k}^{n-k}$ denotes the set of all $(k, n-k)$-shuffles and the symbol $\varepsilon:=\varepsilon\left(X_{1}, X_{2}, \cdots, X_{n}\right)$ denotes the Koszul signs arising from the reordering of the homogeneous objects $X_{1}, X_{2}, \cdots, X_{n}$ in each term of the right hand side.

The differential

$$
\mathcal{L}_{Q}: \Gamma\left(S\left(T_{\mathcal{M}}\right)\right) \rightarrow \Gamma\left(S\left(T_{\mathcal{M}}\right)\right)
$$

is the Lie derivative along the homological vector field $Q$. The induced differential on $\Gamma\left(S\left(T_{\mathcal{M}}\right)\right) \otimes_{\mathcal{R}} \Gamma\left(S\left(T_{\mathcal{M}}\right)\right) \cong \Gamma\left(S\left(T_{\mathcal{M}}\right) \otimes S\left(T_{\mathcal{M}}\right)\right)$ is again the Lie derivative $\mathcal{L}_{Q}$.

The counit map

$$
\epsilon: \Gamma\left(S\left(T_{\mathcal{M}}\right)\right) \rightarrow C^{\infty}(\mathcal{M})
$$

is the canonical projection.

Note that $\Gamma\left(S\left(T_{\mathcal{M}}\right)\right)$ admits a canonical ascending filtration

$$
C^{\infty}(\mathcal{M})=\Gamma\left(S^{\leq 0}\left(T_{\mathcal{M}}\right)\right) \subset \cdots \subset \Gamma\left(S^{\leq n}\left(T_{\mathcal{M}}\right)\right) \subset \cdots .
$$

The following proposition is easily verified.

Proposition 3.3. For any $d g$ manifold $(\mathcal{M}, Q)$, the space $\Gamma\left(S\left(T_{\mathcal{M}}\right)\right)$, equipped with the comultiplication $\Delta$, the differential $\mathcal{L}_{Q}$ and the counit map $\epsilon$ as in (10), (11) and (12), is a filtered dg cocommutative coalgebra over $\left(C^{\infty}(\mathcal{M}), Q\right)$.

3.2. Formal exponential map of a $\mathbf{d g}$ manifold. Let $\mathcal{M}$ be a finite dimensional graded manifold and $\nabla$ be an affine connection on $\mathcal{M}$. A purely algebraic description of the Poincaré-Birkhoff-Witt map has been extended to the context of $\mathbb{Z}$-graded manifolds by Liao-Stiénon [32]. As pointed out in the introduction, for an ordinary smooth manifold, the PBW map is a formal exponential map. In the same way, one can think of the PBW map of a $\mathbb{Z}$-graded manifold as an induced formal exponential map of 'the virtual exponential map'

$$
\exp ^{\nabla}: T_{\mathcal{M}} \rightarrow \mathcal{M} \times \mathcal{M}
$$

by taking fiberwise $\infty$-jets.

Recall that the Poincaré-Birkhoff-Witt map

$$
\operatorname{pbw}^{\nabla}: \Gamma\left(S\left(T_{\mathcal{M}}\right)\right) \rightarrow \mathcal{D}(\mathcal{M})
$$

is defined by the inductive formula [32]:

$$
\begin{aligned}
& \operatorname{pbw}^{\nabla}(f)=f, \quad \forall f \in C^{\infty}(\mathcal{M}) ; \\
& \operatorname{pbw}^{\nabla}(X)=X, \quad \forall X \in \mathfrak{X}(\mathcal{M}) ;
\end{aligned}
$$

and

$$
\operatorname{pbw}^{\nabla}\left(X_{1} \odot \cdots \odot X_{n}\right)=\frac{1}{n} \sum_{k=1}^{n} \varepsilon_{k}\left(X_{k} \operatorname{pbw}^{\nabla}\left(\boldsymbol{X}^{\{k\}}\right)-\operatorname{pbw}^{\nabla}\left(\nabla_{X_{k}} \boldsymbol{X}^{\{k\}}\right)\right),
$$

where $\boldsymbol{X}=X_{1} \odot \cdots \odot X_{n} \in \Gamma\left(S^{n}\left(T_{\mathcal{M}}\right)\right)$ for homogeneous vector fields $X_{1}, \cdots, X_{n} \in \mathfrak{X}(\mathcal{M})$ and $\varepsilon_{k}=(-1)^{\left|X_{k}\right|\left(\left|X_{1}\right|+\cdots+\left|X_{k-1}\right|\right)}$ is the Koszul sign.

Theorem $3.4([32])$. The map $\mathrm{pbw}^{\nabla}$ is an isomorphism of graded coalgebras from $\Gamma\left(S\left(T_{\mathcal{M}}\right)\right)$ to $\mathcal{D}(\mathcal{M})$ over $C^{\infty}(\mathcal{M})$.

Now, we assume there exists a homological vector field $Q$ on $\mathcal{M}$ so that $(\mathcal{M}, Q)$ is a dg manifold. Then, both $\Gamma\left(S\left(T_{\mathcal{M}}\right)\right)$ and $\mathcal{D}(\mathcal{M})$ in $(14)$ are dg coalgebras over $\left(C^{\infty}(\mathcal{M}), Q\right)$, according to Propositions 3.2 and 3.3. We think of the elements of the dg coalgebra $\left(\Gamma\left(S\left(T_{\mathcal{M}}\right)\right), \mathcal{L}_{Q}\right)$ as fiberwise $\mathrm{dg}$ distributions on the dg vector bundle $\pi: T_{\mathcal{M}} \rightarrow \mathcal{M}$ with support the zero section - the homological vector field on $T_{\mathcal{M}}$ is the complete lift $\hat{Q}$ of the homological vector field $Q$ on $\mathcal{M}$ [38,51]. Likewise, 
we think of the elements of the dg coalgebra $\left(\mathcal{D}(\mathcal{M}), \mathcal{L}_{Q}^{\mathcal{D}}\right)$ as fiberwise dg distributions on the dg fiber bundle $\operatorname{pr}_{1}: \mathcal{M} \times \mathcal{M} \rightarrow \mathcal{M}$ with support the diagonal $\Delta$ - the homological vector field on $\mathcal{M} \times \mathcal{M}$ is $(Q, Q)$. On the level of fiberwise $\infty$-jets, the fact that the virtual exponential map (13) is a map of dg manifolds is equivalent to the map pbw ${ }^{\nabla}:\left(\Gamma\left(S\left(T_{\mathcal{M}}\right)\right), \mathcal{L}_{Q}\right) \rightarrow\left(\mathcal{D}(\mathcal{M}), \mathcal{L}_{Q}^{\mathcal{D}}\right)$ being an isomorphism of dg coalgebras over $\left(C^{\infty}(\mathcal{M}), Q\right)$. This consideration leads to the following

Theorem 3.5. Let $(\mathcal{M}, Q)$ be a dg manifold. The Atiyah class $\alpha_{(\mathcal{M}, Q)}$ vanishes if and only if there exists a torsion-free affine connection $\nabla$ on $\mathcal{M}$ such that

$$
\operatorname{pbw}^{\nabla}:\left(\Gamma\left(S\left(T_{\mathcal{M}}\right)\right), \mathcal{L}_{Q}\right) \rightarrow\left(\mathcal{D}(\mathcal{M}), \mathcal{L}_{Q}^{\mathcal{D}}\right)
$$

is an isomorphism of dg coalgebras over $\left(C^{\infty}(\mathcal{M}), Q\right)$.

Remark 3.6. A similar theorem in the same spirit concerning the Atiyah class of Lie pairs was obtained in [31, Theorem 5.10]. It would be interesting to establish a result that encompasses both [31, Theorem 5.10] and Theorem 3.5 under a unified framework.

In order to prove Theorem 3.5, we first introduce a linear map

$$
C^{\nabla}: \Gamma\left(S\left(T_{\mathcal{M}}\right)\right) \rightarrow \mathcal{D}(\mathcal{M})
$$

by

$$
C^{\nabla}:=\mathcal{L}_{Q}^{\mathcal{D}} \circ \mathrm{pbw}^{\nabla}-\mathrm{pbw}^{\nabla} \circ \mathcal{L}_{Q}
$$

One can easily check that $C^{\nabla}$ is a $C^{\infty}(\mathcal{M})$-linear map of degree +1 . Moreover, for $n \geq 0$,

$$
C^{\nabla}\left(\Gamma\left(S^{\leq n}\left(T_{\mathcal{M}}\right)\right)\right) \subseteq \mathcal{D}^{\leq n-1}(\mathcal{M})
$$

The following proposition indicates that $C^{\nabla}$ can be completely determined by a recursive formula.

Proposition 3.7. Let $(\mathcal{M}, Q)$ be a dg manifold, and $\nabla$ a torsion-free affine connection on $\mathcal{M}$. Then the map $C^{\nabla}$ satisfies

$$
\begin{gathered}
C^{\nabla}(f)=0 ; \\
C^{\nabla}(X)=0 ; \\
C^{\nabla}(X \odot Y)=-\operatorname{At}_{(\mathcal{M}, Q)}^{\nabla}(X, Y),
\end{gathered}
$$

for all $f \in C^{\infty}(\mathcal{M}), X, Y \in \mathfrak{X}(\mathcal{M})$, and, for $n \geq 3$, it satisfies the recursive formula

$$
\begin{aligned}
C^{\nabla}(\boldsymbol{X})=\frac{1}{n} \sum_{k=1}^{n} \varepsilon_{k}\left[(-1)^{\left|X_{k}\right|} X_{k}\right. & \left.\cdot C^{\nabla}\left(\boldsymbol{X}^{\{k\}}\right)-C^{\nabla}\left(\nabla_{X_{k}} \boldsymbol{X}^{\{k\}}\right)\right] \\
& -\frac{2}{n} \sum_{i<j} \varepsilon_{i} \varepsilon_{j}(-1)^{\left|X_{i}\right| \cdot\left|X_{j}\right|} \operatorname{pbw}^{\nabla}\left(\operatorname{At}_{(\mathcal{M}, Q)}^{\nabla}\left(X_{i}, X_{j}\right) \odot \boldsymbol{X}^{\{i, j\}}\right),
\end{aligned}
$$

where $\boldsymbol{X}=X_{1} \odot \cdots \odot X_{n} \in \Gamma\left(S^{n}\left(T_{\mathcal{M}}\right)\right)$ denotes the symmetric tensor product of $n$ homogeneous vector fields $X_{1}, \cdots, X_{n} \in \mathfrak{X}(\mathcal{M}) ; \boldsymbol{X}^{\{k\}}=X_{1} \odot \cdots \odot \widehat{X}_{k} \odot \cdots \odot X_{n}$ for any $1 \leq k \leq n$; $\boldsymbol{X}^{\{i, j\}}=X_{1} \odot \cdots \odot \widehat{X}_{i} \odot \cdots \odot \widehat{X}_{j} \odot \cdots \odot X_{n}$ for any $1 \leq i<j \leq n$; and $\varepsilon_{k}=(-1)^{\left|X_{k}\right|\left(\left|X_{1}\right|+\cdots+\left|X_{k-1}\right|\right)}$ is the Koszul sign arising from the reordering $X_{1}, X_{2}, \cdots, X_{n} \mapsto X_{k}, X_{1}, X_{2}, \cdots, X_{k-1}, X_{k+1}, \cdots, X_{n}$.

We now prove Theorem 3.5 based on Proposition 3.7.

Proof of Theorem 3.5. Observe that, according to Proposition 2.5, $\alpha_{(\mathcal{M}, Q)}$ vanishes if and only if there exists an affine connection $\nabla^{\prime}$ for which $\operatorname{At}_{(\mathcal{M}, Q)}^{\nabla^{\prime}}=0$. It follows from $\mathcal{L}_{Q}\left(\nabla^{\prime}\right)=\operatorname{At}_{(\mathcal{M}, Q)}^{\nabla^{\prime}}=0$ that $\mathcal{L}_{Q}\left(T^{\nabla^{\prime}}\right)=0$. Therefore, if the Atiyah cocycle of the affine connection $\nabla^{\prime}$ vanishes, then so 
does the Atiyah cocycle of the torsion-free connection $\nabla=\nabla^{\prime}-\frac{1}{2} T^{\nabla^{\prime}}$ :

$$
\operatorname{At}_{(\mathcal{M}, Q)}^{\nabla}=\mathcal{L}_{Q}(\nabla)=\mathcal{L}_{Q}\left(\nabla^{\prime}-\frac{1}{2} T^{\nabla^{\prime}}\right)=\operatorname{At}_{(\mathcal{M}, Q)}^{\nabla^{\prime}}-\frac{1}{2} \mathcal{L}_{Q}\left(T^{\nabla^{\prime}}\right)=0
$$

Thus, it suffices to prove that $C^{\nabla}=0$ if and only if $\operatorname{At}_{(\mathcal{M}, Q)}^{\nabla}=0$.

Assume that $C^{\nabla}=0$. By Proposition 3.7, we have

$$
\operatorname{At}_{(\mathcal{M}, Q)}^{\nabla}(X, Y)=-C^{\nabla}(X \odot Y)=0
$$

for all $X, Y \in \mathfrak{X}(\mathcal{M})$.

Conversely, assume that $\operatorname{At}_{(\mathcal{M}, Q)}^{\nabla}=0$. Then we have $C^{\nabla}(X \odot Y)=0$ by Proposition 3.7. Hence $C^{\nabla}(\boldsymbol{Y})=0$ for all $\boldsymbol{Y} \in \Gamma\left(S^{\leq 2}\left(T_{\mathcal{M}}\right)\right)$. Moreover, Equation (21) can be written as

$$
C^{\nabla}(\boldsymbol{X})=\frac{1}{n} \sum_{k=1}^{n} \varepsilon_{k}\left[(-1)^{\left|X_{k}\right|} X_{k} \cdot C^{\nabla}\left(\boldsymbol{X}^{\{k\}}\right)-C^{\nabla}\left(\nabla_{X_{k}} \boldsymbol{X}^{\{k\}}\right)\right], \quad \forall \boldsymbol{X} \in\left(\Gamma\left(S^{\geq 3}\left(T_{\mathcal{M}}\right)\right)\right) .
$$

Therefore, $C^{\nabla}=0$ by the inductive argument.

3.3. Proof of Proposition 3.7. Now we turn to the proof of Proposition 3.7. We will divide the proof into several lemmas.

Lemma 3.8. Under the same hypothesis as in Proposition 3.7, Equations (18), (19) and (20) hold.

Proof. Equations (18) and (19) follow immediately from Equation (15).

To prove Equation $(20)$, let $X, Y \in \mathfrak{X}(\mathcal{M})$ be homogeneous vector fields. Since $\nabla$ is torsion-free, we have

$$
\nabla_{X} Y-(-1)^{|X| \cdot|Y|} \nabla_{Y} X=[X, Y]=X Y-Y X .
$$

It then follows from Equation (16) that

$$
\operatorname{pbw}^{\nabla}(X \odot Y)=X Y-\nabla_{X} Y \text {. }
$$

From there, we obtain

$$
\mathcal{L}_{Q}^{\mathcal{D}} \circ \operatorname{pbw}^{\nabla}(X \odot Y)=[Q, X] Y+(-1)^{|X|} X[Q, Y]-\left[Q, \nabla_{X} Y\right]
$$

and

$$
\begin{aligned}
\operatorname{pbw}^{\nabla} \circ \mathcal{L}_{Q}(X \odot Y) & =\operatorname{pbw}^{\nabla}\left([Q, X] \odot Y+(-1)^{|X|} X \odot[Q, Y]\right) \\
& =\left([Q, X] Y-\nabla_{[Q, X]} Y\right)+(-1)^{|X|}\left(X[Q, Y]-\nabla_{X}[Q, Y]\right) .
\end{aligned}
$$

As a result, we have

$$
\begin{aligned}
C^{\nabla}(X \odot Y) & =\left(\mathcal{L}_{Q}^{\mathcal{D}} \circ \mathrm{pbw}^{\nabla}-\mathrm{pbw}^{\nabla} \circ \mathcal{L}_{Q}\right)(X \odot Y) \\
& =-\left(\left[Q, \nabla_{X} Y\right]-\nabla_{[Q, X]} Y-(-1)^{|X|} \nabla_{X}[Q, Y]\right) \\
& =-\operatorname{At}_{(\mathcal{M}, Q)}^{\nabla}(X, Y) .
\end{aligned}
$$

In the sequel, we adopt the following notations. For any $\boldsymbol{X}=X_{1} \odot \cdots \odot X_{n} \in \Gamma\left(S^{n}\left(T_{\mathcal{M}}\right)\right)$, we write $\boldsymbol{X}^{\{k\}}=X_{1} \odot \cdots \odot \widehat{X}_{k} \odot \cdots \odot X_{n}$; for $i \neq j$, we write $\boldsymbol{X}^{\{i, j\}}=X_{1} \odot \cdots \odot \widehat{X}_{i} \odot \cdots \odot \widehat{X}_{j} \odot \cdots \odot X_{n}$, and for all $1 \leq i \leq n, \boldsymbol{X}^{\{i, i\}}=0$. 
Lemma 3.9. Under the same hypothesis as in Proposition 3.7, for all $\boldsymbol{X}=X_{1} \odot \cdots \odot X_{n} \in \Gamma\left(S^{n}\left(T_{\mathcal{M}}\right)\right)$ with $n \geq 3$, we have

$$
\begin{aligned}
\mathcal{L}_{Q}^{\mathcal{D}} \circ \operatorname{pbw}^{\nabla}(\boldsymbol{X})= & \frac{1}{n} \sum_{k=1}^{n} \varepsilon \cdot\left[Q, X_{k}\right] \cdot \operatorname{pbw}^{\nabla}\left(\boldsymbol{X}^{\{k\}}\right) \\
& +\frac{1}{n} \sum_{k=1}^{n}\left(\varepsilon \cdot X_{k} \cdot \mathcal{L}_{Q}^{\mathcal{D}}\left(\operatorname{pbw}^{\nabla}\left(\boldsymbol{X}^{\{k\}}\right)\right)-\varepsilon \cdot \mathcal{L}_{Q}^{\mathcal{D}}\left(\operatorname{pbw}^{\nabla}\left(\nabla_{X_{k}} \boldsymbol{X}^{\{k\}}\right)\right)\right)
\end{aligned}
$$

and

$$
\begin{aligned}
& \operatorname{pbw}^{\nabla} \circ \mathcal{L}_{Q}(\boldsymbol{X}) \\
& =\frac{1}{n} \sum_{k=1}^{n}\left(\varepsilon \cdot\left[Q, X_{k}\right] \cdot \operatorname{pbw}^{\nabla}\left(\boldsymbol{X}^{\{k\}}\right)+\varepsilon \cdot X_{k} \cdot \operatorname{pbw}^{\nabla}\left(\mathcal{L}_{Q}\left(\boldsymbol{X}^{\{k\}}\right)\right)-\varepsilon \cdot \mathrm{pbw}^{\nabla}\left(\mathcal{L}_{Q}\left(\nabla_{X_{k}} \boldsymbol{X}^{\{k\}}\right)\right)\right) \\
& \quad+\frac{1}{n} \sum_{i<j} \varepsilon \cdot \operatorname{pbw}^{\nabla}\left(2 \operatorname{At}_{(\mathcal{M}, Q)}^{\nabla}\left(X_{i}, X_{j}\right) \odot \boldsymbol{X}^{\{i, j\}}\right) .
\end{aligned}
$$

In the two equations above and in the proof of the Lemma as well, the symbol $\varepsilon=\varepsilon\left(Q, X_{1}, \cdots, X_{n}\right)$ denotes the Koszul signs arising from the reordering of the homogeneous objects $Q, X_{1}, \cdots, X_{n}$ in each term of the right hand sides.

Proof. The formula for $\mathcal{L}_{Q}^{\mathcal{D}} \circ \mathrm{pbw}^{\nabla}(\boldsymbol{X})$ is immediate from Equation (16).

Next, we will compute pbw ${ }^{\nabla} \circ \mathcal{L}_{Q}(\boldsymbol{X})$. Since $\mathcal{L}_{Q}(\boldsymbol{X})=\sum_{k=1}^{n} \varepsilon \cdot\left(\left[Q, X_{k}\right] \odot \boldsymbol{X}^{\{k\}}\right)$, applying Equation (16), we have

where

$$
\operatorname{pbw}^{\nabla} \circ \mathcal{L}_{Q}(\boldsymbol{X})=\frac{1}{n}\left(\mathcal{A}^{1}-\mathcal{A}^{2}+\mathcal{B}-\mathcal{C}\right)
$$

$$
\begin{aligned}
\mathcal{A}^{1} & :=\sum_{k=1}^{n} \varepsilon \cdot\left[Q, X_{k}\right] \cdot \operatorname{pbw}^{\nabla}\left(\boldsymbol{X}^{\{k\}}\right), \\
\mathcal{A}^{2} & :=\sum_{k=1}^{n} \varepsilon \cdot \operatorname{pbw}^{\nabla}\left(\nabla_{\left[Q, X_{k}\right]} \boldsymbol{X}^{\{k\}}\right), \\
\mathcal{B} & :=\sum_{k=1}^{n} \sum_{i=1}^{n} \varepsilon \cdot X_{i} \cdot \operatorname{pbw}^{\nabla}\left(\left[Q, X_{k}\right] \odot \boldsymbol{X}^{\{i, k\}}\right), \\
\mathcal{C} & :=\sum_{k=1}^{n} \sum_{i=1}^{n} \varepsilon \cdot \operatorname{pbw}^{\nabla}\left(\nabla_{X_{i}}\left(\left[Q, X_{k}\right] \odot \boldsymbol{X}^{\{i, k\}}\right) .\right.
\end{aligned}
$$

First, by changing the order of summation, we obtain

$$
\begin{aligned}
\mathcal{B} & =\sum_{i=1}^{n} \sum_{k=1}^{n} \varepsilon \cdot X_{i} \cdot \operatorname{pbw}^{\nabla}\left(\left[Q, X_{k}\right] \odot \boldsymbol{X}^{\{i, k\}}\right) \\
& =\sum_{i=1}^{n} \varepsilon \cdot X_{i} \cdot \operatorname{pbw}^{\nabla}\left(\mathcal{L}_{Q}\left(\boldsymbol{X}^{\{i\}}\right)\right) .
\end{aligned}
$$


We also can write

$$
\begin{aligned}
\mathcal{A}^{2} & =\sum_{k=1}^{n} \sum_{i=1}^{n} \varepsilon \cdot \mathrm{pbw}^{\nabla}\left(\left(\nabla_{\left[Q, X_{k}\right]} X_{i}\right) \odot \boldsymbol{X}^{\{k, i\}}\right) \\
& =\sum_{k=1}^{n} \sum_{i=1}^{n} \varepsilon \cdot \mathrm{pbw}^{\nabla}\left(\left(\nabla_{\left[Q, X_{i}\right]} X_{k}\right) \odot \boldsymbol{X}^{\{i, k\}}\right) .
\end{aligned}
$$

Now we also have

$$
\begin{aligned}
& \sum_{k=1}^{n} \sum_{i=1}^{n} \varepsilon \cdot \mathrm{pbw}^{\nabla}\left(\left[Q, X_{k}\right] \odot \nabla_{X_{i}} \boldsymbol{X}^{\{i, k\}}\right) \\
= & \sum_{k=1}^{n} \sum_{i=1}^{n} \sum_{j=1}^{n} \varepsilon \cdot \mathrm{pbw}^{\nabla}\left(\left[Q, X_{k}\right] \odot \nabla_{X_{i}} X_{j} \odot \boldsymbol{X}^{\{i, k, j\}}\right) \\
= & \sum_{k=1}^{n} \sum_{i=1}^{n} \sum_{j=1}^{n} \varepsilon \cdot \mathrm{pbw}^{\nabla}\left(\nabla_{X_{i}} X_{j} \odot\left[Q, X_{k}\right] \odot \boldsymbol{X}^{\{i, k, j\}}\right) \\
= & \sum_{i=1}^{n} \sum_{j=1}^{n} \varepsilon \cdot \mathrm{pbw}^{\nabla}\left(\nabla_{X_{i}} X_{j} \odot \mathcal{L}_{Q} \boldsymbol{X}^{\{i, j\}}\right) \\
= & \sum_{i=1}^{n} \sum_{k=1}^{n} \varepsilon \cdot \mathrm{pbw}^{\nabla}\left(\nabla_{X_{i}} X_{k} \odot \mathcal{L}_{Q} \boldsymbol{X}^{\{i, k\}}\right) .
\end{aligned}
$$

Therefore, it follows that

$$
\begin{aligned}
& \sum_{k=1}^{n} \sum_{i=1}^{n} \varepsilon \cdot \operatorname{pbw}^{\nabla}\left(\left[Q, \nabla_{X_{i}} X_{k}\right] \odot \boldsymbol{X}^{\{i, k\}}\right)+\sum_{k=1}^{n} \sum_{i=1}^{n} \varepsilon \cdot \mathrm{pbw}^{\nabla}\left(\left[Q, X_{k}\right] \odot \nabla_{X_{i}} \boldsymbol{X}^{\{i, k\}}\right) \\
= & \sum_{k=1}^{n} \sum_{i=1}^{n} \varepsilon \cdot \operatorname{pbw}^{\nabla}\left(\left[Q, \nabla_{X_{i}} X_{k}\right] \odot \boldsymbol{X}^{\{i, k\}}\right)+\sum_{i=1}^{n} \sum_{k=1}^{n} \varepsilon \cdot \operatorname{pbw}^{\nabla}\left(\nabla_{X_{i}} X_{k} \odot \mathcal{L}_{Q} \boldsymbol{X}^{\{i, k\}}\right) \\
= & \sum_{i=1}^{n} \sum_{k=1}^{n} \varepsilon \cdot \operatorname{pbw}^{\nabla} \mathcal{L}_{Q}\left(\nabla_{X_{i}} X_{k} \odot \boldsymbol{X}^{\{i, k\}}\right) \\
= & \sum_{i=1}^{n} \varepsilon \cdot \operatorname{pbw}^{\nabla}\left(\mathcal{L}_{Q}\left(\nabla_{X_{i}} X^{\{i\}}\right)\right) .
\end{aligned}
$$

Moreover,

$$
\mathcal{C}=\sum_{k=1}^{n} \sum_{i=1}^{n} \varepsilon \cdot \operatorname{pbw}^{\nabla}\left(\left(\nabla_{X_{i}}\left[Q, X_{k}\right]\right) \odot \boldsymbol{X}^{\{i, k\}}\right)+\sum_{k=1}^{n} \sum_{i=1}^{n} \varepsilon \cdot \mathrm{pbw}^{\nabla}\left(\left[Q, X_{k}\right] \odot \nabla_{X_{i}} \boldsymbol{X}^{\{i, k\}}\right) .
$$


Then by combining Equations (24), (25) and (26) and using the definition of Atiyah cocycles, we obtain

$$
\begin{aligned}
\mathcal{A}^{2}+\mathcal{C}= & \sum_{k=1}^{n} \sum_{i=1}^{n} \varepsilon \cdot \operatorname{pbw}^{\nabla}\left(\left(\left[Q, \nabla_{X_{i}} X_{k}\right]-\operatorname{At}_{(\mathcal{M}, Q)}^{\nabla}\left(X_{i}, X_{k}\right)\right) \odot \boldsymbol{X}^{\{i, k\}}\right) \\
& +\sum_{k=1}^{n} \sum_{i=1}^{n} \varepsilon \cdot \operatorname{pbw}^{\nabla}\left(\left[Q, X_{k}\right] \odot \nabla_{X_{i}} \boldsymbol{X}^{\{i, k\}}\right) \\
= & \sum_{i=1}^{n} \varepsilon \cdot \operatorname{pbw}^{\nabla}\left(\mathcal{L}_{Q}\left(\nabla_{X_{i}} X^{\{i\}}\right)\right)-\sum_{i<j} \varepsilon \cdot \operatorname{pbw}^{\nabla}\left(2 \operatorname{At}_{(\mathcal{M}, Q)}^{\nabla}\left(X_{i}, X_{j}\right) \odot \boldsymbol{X}^{\{i, j\}}\right) .
\end{aligned}
$$

The conclusion thus follows from Equations (22), (23), and (27).

Proof of Proposition 3.7. Equations (18), (19) and (20) have been proved in Lemma 3.8. It remains to prove Equation (21). According to Lemma 3.9, we have

$$
\begin{aligned}
\mathcal{L}_{Q}^{\mathcal{D}} \circ \operatorname{pbw}^{\nabla}(\boldsymbol{X})-\mathrm{pbw}^{\nabla} \circ \mathcal{L}_{Q}(\boldsymbol{X})= & \frac{1}{n} \sum_{k=1}^{n} \varepsilon_{k}(-1)^{\left|X_{k}\right|} X_{k} \cdot\left(\mathcal{L}_{Q}^{\mathcal{D}} \circ \mathrm{pbw}^{\nabla}-\mathrm{pbw}^{\nabla} \circ \mathcal{L}_{Q}\right)\left(\boldsymbol{X}^{\{k\}}\right) \\
& -\frac{1}{n} \sum_{k=1}^{n} \varepsilon_{k}\left(\mathcal{L}_{Q}^{\mathcal{D}} \circ \mathrm{pbw}^{\nabla}-\mathrm{pbw}^{\nabla} \circ \mathcal{L}_{Q}\right)\left(\nabla_{X_{k}} \boldsymbol{X}^{\{k\}}\right) \\
& -\frac{1}{n} \sum_{i<j} \varepsilon_{i} \varepsilon_{j}(-1)^{\left|X_{i}\right| \cdot\left|X_{j}\right|} \mathrm{pbw}^{\nabla}\left(2 \operatorname{At}_{(\mathcal{M}, Q)}\left(X_{i}, X_{j}\right) \odot \boldsymbol{X}^{\{i, j\}}\right) .
\end{aligned}
$$

This concludes the proof of Proposition 3.7.

\section{Atiyah Class and homotopy Lie algebras}

This section is devoted to the study of homotopy Lie algebras associated to the Atiyah class of dg manifolds.

4.1. Kapranov $L_{\infty}[1]$ algebras of $\mathbf{d g}$ manifolds. The Atiyah class of a holomorphic vector bundle is closely related to $L_{\infty}$ [1] algebras as shown by the pioneer work of Kapranov $[25,42,43]$. These $L_{\infty}$ [1] algebras play an important role in derived geometry [10,36, 42] and construction of Rozansky-Witten invariants [25, 27, 43, 45, 53].

In this section, following Kapranov [25], we show that the Atiyah class of a dg manifold is related to $L_{\infty}[1]$ algebras in a similar fashion. We refer to [17, Sections 4 and 5] for the interpretation in terms of derived category.

Let $(\mathcal{M}, Q)$ be a dg manifold and let $\nabla$ be an affine connection on $\mathcal{M}$. The Lie derivative $\mathcal{L}_{Q}^{\mathcal{D}}$ along the homological vector field $Q$ is a degree +1 coderivation of the $\operatorname{dg}$ coalgebra $\mathcal{D}(\mathcal{M})$ over $\left(C^{\infty}(\mathcal{M}), Q\right)$ according to Proposition 3.2.

Transferring $\mathcal{L}_{Q}^{\mathcal{D}}$ from $\mathcal{D}(\mathcal{M})$ to $\Gamma\left(S\left(T_{\mathcal{M}}\right)\right.$ ) by the graded coalgebra isomorphism pbw ${ }^{\nabla}$ (14), we obtain a degree +1 coderivation $\delta^{\nabla}$ of $\Gamma\left(S\left(T_{\mathcal{M}}\right)\right)$ :

$$
\delta^{\nabla}:=\left(\mathrm{pbw}^{\nabla}\right)^{-1} \circ \mathcal{L}_{Q}^{\mathcal{D}} \circ \mathrm{pbw}^{\nabla} .
$$

Therefore

$$
\left(\Gamma\left(S\left(T_{\mathcal{M}}\right)\right), \delta^{\nabla}\right)
$$

is a $\mathrm{dg}$ coalgebra over the $\mathrm{dg} \operatorname{ring}\left(C^{\infty}(\mathcal{M}), Q\right)$. 
Finally, dualizing $\delta^{\nabla}$ over $\left(C^{\infty}(\mathcal{M}), Q\right)$, we obtain a degree +1 derivation:

$$
D^{\nabla}: \Gamma\left(\widehat{S}\left(T_{\mathcal{M}}^{\vee}\right)\right) \rightarrow \Gamma\left(\widehat{S}\left(T_{\mathcal{M}}^{\vee}\right)\right)
$$

Here we used the identification $\Gamma\left(\widehat{S}\left(T_{\mathcal{M}}^{\vee}\right)\right) \cong \operatorname{Hom}_{C^{\infty}(\mathcal{M})}\left(\Gamma\left(S\left(T_{\mathcal{M}}\right)\right), C^{\infty}(\mathcal{M})\right)$.

The following theorem was first announced in [38], but a proof was omitted. We will present a complete proof below.

Theorem 4.1. Let $(\mathcal{M}, Q)$ be a dg manifold, and let $\nabla$ be a torsion-free affine connection on $\mathcal{M}$.

(i) The operator $D^{\nabla}$ is a derivation of degree +1 of the graded algebra $\Gamma\left(\widehat{S}\left(T_{\mathcal{M}}^{\vee}\right)\right)$ satisfying $\left(D^{\nabla}\right)^{2}=0$. Thus $\left(\Gamma\left(\widehat{S}\left(T_{\mathcal{M}}^{\vee}\right)\right), D^{\nabla}\right)$ is a dg algebra.

(ii) There exists a sequence of degree +1 sections $R_{k} \in \Gamma\left(S^{k}\left(T_{\mathcal{M}}^{\vee}\right) \otimes T_{\mathcal{M}}\right), k \geqslant 2$ whose first term $R_{2}$ equals to $-\operatorname{At}_{(\mathcal{M}, Q)}^{\nabla}$, such that

$$
D^{\nabla}=\mathcal{L}_{Q}+\sum_{k=2}^{\infty} \widetilde{R_{k}}
$$

where each $\widetilde{R_{k}}: \Gamma\left(\widehat{S}\left(T_{\mathcal{M}}^{\vee}\right)\right) \rightarrow \Gamma\left(\widehat{S}\left(T_{\mathcal{M}}^{\vee}\right)\right)$ denotes the $\mathcal{R}$-linear degree +1 derivation corresponding to $R_{k}$.

(iii) Different choices of torsion-free affine connections $\nabla$ induce isomorphic $d g$ algebras $\left(\Gamma\left(\widehat{S}\left(T_{\mathcal{M}}^{\vee}\right)\right), D^{\nabla}\right)$.

Remark 4.2. The graded algebra $\Gamma\left(\widehat{S}\left(T_{\mathcal{M}}^{\vee}\right)\right)$ can be thought of as the graded algebra of functions on a graded manifold $\widetilde{T}_{\mathcal{M}}$ with support $M$ and $D^{\nabla}$ as a homological vector field on $\widetilde{T}_{\mathcal{M}}$. Note that $T_{\mathcal{M}}$ and $\widetilde{T}_{\mathcal{M}}$ are different graded manifolds: the support of $T_{\mathcal{M}}$ is $T_{M}$ while the support of $\widetilde{T}_{\mathcal{M}}$ is $M$.

Before we prove this theorem, we need to recall some basic notations.

Recall that given a graded commutative algebra $\mathcal{R}$ and a graded $\mathcal{R}$-module $V$, the symmetric tensor algebra $\left(S_{\mathcal{R}}(V), \mu\right)$ over $\mathcal{R}$ admits a canonical graded coalgebra structure $\Delta: S_{\mathcal{R}}(V) \rightarrow S_{\mathcal{R}}(V) \otimes_{\mathcal{R}} S_{\mathcal{R}}(V)$ defined by [31]

$$
\begin{aligned}
\Delta\left(v_{1} \odot \cdots \odot v_{n}\right)= & 1 \otimes\left(v_{1} \odot \cdots \odot v_{n}\right)+\left(v_{1} \odot \cdots \odot v_{n}\right) \otimes 1 \\
& +\sum_{k=1}^{n-1} \sum_{\sigma \in \mathfrak{S}_{k}^{n-k}} \varepsilon \cdot\left(v_{\sigma(1)} \odot \cdots \odot v_{\sigma(k)}\right) \otimes\left(v_{\sigma(k+1)} \odot \cdots \odot v_{\sigma(n)}\right)
\end{aligned}
$$

for homogeneous elements $v_{1}, \cdots, v_{n} \in V$. Here the symbol $\varepsilon=\varepsilon\left(v_{1}, v_{2}, \cdots, v_{n}\right)$ denotes the Koszul signs arising from the reordering of the homogeneous objects $v_{1}, v_{2}, \cdots, v_{n}$ in each term of the right hand side.

The following lemma is standard - see, for example, [35, 31].

Lemma 4.3. Let $\mathcal{R}$ be a graded commutative algebra and $V$ be an $\mathcal{R}$-module. There is a natural isomorphism

$$
\operatorname{coDer}_{\mathcal{R}}\left(S_{\mathcal{R}}(V), S_{\mathcal{R}}(V)\right) \stackrel{\simeq}{\rightarrow} \prod_{k=0}^{\infty} \operatorname{Hom}_{\mathcal{R}}\left(S_{\mathcal{R}}^{k}(V), V\right)
$$

as $\mathcal{R}$-modules. 
More explicitly, the correspondence between a sequence of maps $\left\{q_{k}\right\}_{k \geq 0}$ with $q_{k} \in \operatorname{Hom}_{\mathcal{R}}\left(S_{\mathcal{R}}^{k}(V), V\right)$ and a coderivation $Q \in \operatorname{coDer}_{\mathcal{R}}\left(S_{\mathcal{R}}(V), S_{\mathcal{R}}(V)\right)$ is given by

$$
\begin{aligned}
Q\left(v_{1} \odot \cdots \odot v_{n}\right)= & q_{0}(1) \odot v_{1} \odot \cdots \odot v_{n}+q_{n}\left(v_{1} \odot \cdots \odot v_{n}\right) \odot 1 \\
& +\sum_{k=1}^{n-1} \sum_{\sigma \in \mathfrak{S}_{k}^{n-k}} \varepsilon \cdot q_{k}\left(v_{\sigma(1)} \odot \cdots \odot v_{\sigma(k)}\right) \odot v_{\sigma(k+1)} \odot \cdots \odot v_{\sigma(n)}
\end{aligned}
$$

for homogeneous vectors $v_{1}, \cdots, v_{n} \in V$.

For a given graded $\mathcal{R}$-coalgebra $(C, \Delta)$ and a graded $\mathcal{R}$-algebra $(A, \mu)$, the convolution product $\star$ on the graded vector space $\operatorname{Hom}_{\mathcal{R}}(C, A)$ is defined by

$$
f \star g=\mu \circ(f \otimes g) \circ \Delta
$$

$\forall f, g \in \operatorname{Hom}_{\mathcal{R}}(C, A)$. It is clear that $\left(\operatorname{Hom}_{\mathcal{R}}(C, A), \star\right)$ is a graded $\mathcal{R}$-algebra. In particular, since $S_{\mathcal{R}}(V)$ is both a graded coalgebra and a graded algebra, the space of $\mathcal{R}$-linear maps $\operatorname{Hom}_{\mathcal{R}}\left(S_{\mathcal{R}}(V), S_{\mathcal{R}}(V)\right)$ admits a convolution product:

$$
(f \star g)(\mathbf{v})=\sum_{(\mathbf{v})}(-1)^{|g| \cdot\left|\mathbf{v}_{(1)}\right|} f\left(\mathbf{v}_{(1)}\right) \odot g\left(\mathbf{v}_{(2)}\right),
$$

where $\mathbf{v} \in S_{\mathcal{R}}(V)$ and $\Delta(\mathbf{v})=\sum_{(\mathbf{v})} \mathbf{v}_{(1)} \otimes \mathbf{v}_{(2)}$.

Using the above notation (32), we may write Equation (31) as

$$
Q=\sum_{k=0}^{\infty}\left(\bar{q}_{k} \star \operatorname{id}_{S_{\mathcal{R}}(V)}\right),
$$

where the map $\bar{q}_{k}: S_{\mathcal{R}}(V) \rightarrow S_{\mathcal{R}}(V)$ is defined by the following commutative diagram:

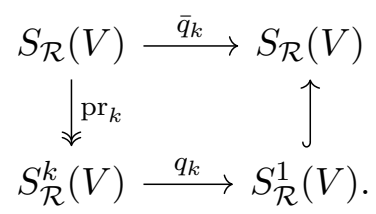

Here $\operatorname{pr}_{k}: S_{\mathcal{R}}(V) \rightarrow S_{\mathcal{R}}^{k}(V)$ denotes the canonical projection. We write id for $\operatorname{id}_{S_{\mathcal{R}}(V)}$ below if there is no confusion. We are now ready to give a detailed proof of Theorem 4.1.

Proof of Theorem 4.1. For (i), by construction, it is clear that the operator $D^{\nabla}$ in (30) is indeed a degree +1 derivation. Since $Q$ is a homological vector field, from (28), it follows that $\left(\delta^{\nabla}\right)^{2}=0$. Therefore $\left(D^{\nabla}\right)^{2}=0$.

To prove (ii), consider the case when $\mathcal{R}=C^{\infty}(\mathcal{M})$ and $V=\Gamma\left(T_{\mathcal{M}}\right)$ in Lemma 4.3. Recall that $C^{\nabla}$ in (17) is $\mathcal{R}$-linear, and pbw ${ }^{\nabla}: \Gamma\left(S\left(T_{\mathcal{M}}\right)\right) \rightarrow \mathcal{D}(\mathcal{M})$ is an isomorphism of graded coalgebras over $\mathcal{R}$. Since $\mathcal{L}_{Q} \in \operatorname{coDer}_{\mathcal{R}}\left(\Gamma\left(S\left(T_{\mathcal{M}}\right)\right)\right)$ and $\mathcal{L}_{Q}^{\mathcal{D}} \in \operatorname{coDer}_{\mathcal{R}}(\mathcal{D}(\mathcal{M}))$, it thus follows that

$$
\left(\mathrm{pbw}^{\nabla}\right)^{-1} \circ C^{\nabla}=\left(\mathrm{pbw}^{\nabla}\right)^{-1} \circ \mathcal{L}_{Q}^{\mathcal{D}} \circ \mathrm{pbw}^{\nabla}-\mathcal{L}_{Q} \in \operatorname{coDer}_{\mathcal{R}}\left(\Gamma\left(S\left(T_{\mathcal{M}}\right)\right)\right) .
$$

Since both $\mathcal{L}_{Q}^{\mathcal{D}}$ and $\mathcal{L}_{Q}$ are of degree +1 and $\mathrm{pbw}^{\nabla}$ is of degree 0 , it follows from Lemma 4.3 and Equation (33) that there exists a sequence of degree +1 sections $R_{k} \in \Gamma\left(S^{k}\left(T_{\mathcal{M}}^{\vee}\right) \otimes T_{\mathcal{M}}\right), k \geqslant 0$, such that

$$
\left(\mathrm{pbw}^{\nabla}\right)^{-1} \circ \mathcal{L}_{Q}^{\mathcal{D}} \circ \mathrm{pbw}^{\nabla}-\mathcal{L}_{Q}=\sum_{k=0}^{\infty}\left(\bar{R}_{k} \star \mathrm{id}\right) .
$$

Here we think of $R_{k}$ as an $\mathcal{R}$-linear map $R_{k}: \Gamma\left(S^{k}\left(T_{\mathcal{M}}\right)\right) \rightarrow \Gamma\left(T_{\mathcal{M}}\right)$ and $\bar{R}_{k}: \Gamma\left(S\left(T_{\mathcal{M}}\right)\right) \rightarrow \Gamma\left(S\left(T_{\mathcal{M}}\right)\right)$ defined as in Diagram (34). 
From Equations (18), (19) and (20), it follows that

$$
R_{0}=0, \quad R_{1}=0, \quad \text { and } \quad R_{2}=-\operatorname{At}_{(\mathcal{M}, Q)}^{\nabla} .
$$

Thus the conclusion follows immediately from (35) by taking its $\mathcal{R}$-dual.

Finally, assume that $\nabla^{\prime}$ is another torsion-free affine connection. Let $\phi:=\left(\mathrm{pbw}^{\nabla^{\prime}}\right)^{-1} \circ \mathrm{pbw}^{\nabla}$. Then from Proposition 3.2, Proposition 3.3 and Theorem 3.4, it follows that

$$
\phi:\left(\Gamma\left(S\left(T_{\mathcal{M}}\right)\right), \delta^{\nabla}\right) \stackrel{\cong}{\rightarrow}\left(\Gamma\left(S\left(T_{\mathcal{M}}\right)\right), \delta^{\nabla^{\prime}}\right)
$$

is an isomorphism of $\mathrm{dg}$ coalgebras over $\left(C^{\infty}(\mathcal{M}), Q\right)$. By dualizing it over the dg algebra $\left(C^{\infty}(\mathcal{M}), Q\right)$, we have that

$$
\phi^{T}:\left(\Gamma\left(\widehat{S}\left(T_{\mathcal{M}}^{\vee}\right)\right), D^{\nabla^{\prime}}\right) \cong\left(\Gamma\left(\widehat{S}\left(T_{\mathcal{M}}^{\vee}\right)\right), D^{\nabla}\right)
$$

is an isomorphism of dg algebras over $\left(C^{\infty}(\mathcal{M}), Q\right)$. This concludes the proof of the theorem.

Indeed, following Kapranov [25], one may consider $\left(\Gamma\left(\widehat{S}\left(T_{\mathcal{M}}^{\vee}\right)\right), D^{\nabla}\right)$ as the 'dg algebra of functions' on the 'formal neighborhood' of the diagonal $\Delta$ of the product dg manifold $(\mathcal{M} \times \mathcal{M},(Q, Q))$ : the PBW map pbw ${ }^{\nabla}$ is, by construction, a formal exponential map identifying a 'formal neighborhood' of the zero section of $T_{\mathcal{M}}$ to a 'formal neighborhood' of the diagonal of $\mathcal{M} \times \mathcal{M}$ as $\mathbb{Z}$-graded manifolds and Equation (28) asserts that $D^{\nabla}$ is the homological vector field obtained on $T_{\mathcal{M}}$ by pullback of the vector field $(Q, Q)$ on $\mathcal{M} \times \mathcal{M}$ through this formal exponential map. The readers are invited to compare Theorem 4.1 with [25, Theorem 2.8.2].

As an immediate consequence, we are ready to prove the main result of this section.

Theorem 4.4. Let $(\mathcal{M}, Q)$ be a dg manifold. Each choice of an affine connection $\nabla$ on $\mathcal{M}$ determines an $L_{\infty}[1]$ algebra structure on the space of vector fields $\mathfrak{X}(\mathcal{M})$. While the unary bracket $\lambda_{1}: S^{1}(\mathfrak{X}(\mathcal{M})) \rightarrow \mathfrak{X}(\mathcal{M})$ is the Lie derivative $\mathcal{L}_{Q}$ along the homological vector field, the higher multibrackets $\lambda_{k}: S^{k}(\mathfrak{X}(\mathcal{M})) \rightarrow \mathfrak{X}(\mathcal{M})$, with $k \geq 2$, arise as the composition

$$
\lambda_{k}: S^{k}(\mathfrak{X}(\mathcal{M})) \rightarrow \Gamma\left(S^{k}\left(T_{\mathcal{M}}\right)\right) \stackrel{R_{k}}{\rightarrow} \mathfrak{X}(\mathcal{M})
$$

induced by a family of sections $\left\{R_{k}\right\}_{k \geq 2}$ of the vector bundles $S^{k}\left(T_{\mathcal{M}}^{\vee}\right) \otimes T_{\mathcal{M}}$ starting with $R_{2}=-\operatorname{At}_{(\mathcal{M}, Q)}^{\nabla}$.

Furthermore, the $L_{\infty}[1]$ algebra structures on $\mathfrak{X}(\mathcal{M})$ arising from different choices of affine connections are all isomorphic.

For clarity, we point out that $S^{k}(\mathfrak{X}(\mathcal{M}))$ denotes the symmetric tensor product over the field $\mathbb{K}$ of $k$ copies of $\mathfrak{X}(\mathcal{M})$. While $\lambda_{1}$ is merely a $\mathbb{K}$-linear endomorphism of $\mathfrak{X}(\mathcal{M})$, we note that, for all $k \geq 2$, the multibracket $\lambda_{k}$ is $C^{\infty}(\mathcal{M})$-linear in each of its $k$ arguments.

Proof. The first part follows immediately from the fact that $\left(\Gamma\left(S\left(T_{\mathcal{M}}\right)\right), \delta^{\nabla}\right)$ as in $(29)$ is a dg coalgebra over $\left(C^{\infty}(\mathcal{M}), Q\right)$.

The uniqueness is a direct consequence of Theorem 4.1 as well. Indeed, it is easier to derive it using the dg coalgebra $\left(\Gamma\left(S\left(T_{\mathcal{M}}\right)\right), \delta^{\nabla}\right)$ as in $(29)$. If $\nabla^{\prime}$ is another torsion-free affine connection on $\mathcal{M}$, we know that $\phi:\left(\Gamma\left(S\left(T_{\mathcal{M}}\right)\right), \delta^{\nabla}\right) \cong\left(\Gamma\left(S\left(T_{\mathcal{M}}\right)\right), \delta^{\nabla^{\prime}}\right)$ as in (37) is an isomorphism of dg coalgebras over the $\left(C^{\infty}(\mathcal{M}), Q\right)$. Thus it follows that the sequence of maps $\left\{\phi_{k}\right\}_{k \geq 1}$ defined by the composition

$$
\phi_{k}: S^{k}(\mathfrak{X}(\mathcal{M})) \rightarrow \Gamma\left(S^{k}\left(T_{\mathcal{M}}\right)\right) \stackrel{\phi}{\rightarrow} \Gamma\left(S\left(T_{\mathcal{M}}\right)\right) \stackrel{\operatorname{pr}_{1}}{\rightarrow} \Gamma\left(T_{\mathcal{M}}\right)=\mathfrak{X}(\mathcal{M})
$$

is an isomorphism of $L_{\infty}[1]$ algebras. Indeed, it is simple to see from (15) that the linear term $\phi_{1}$ is the identity map. 
Such an $L_{\infty}[1]$ algebra on $\mathfrak{X}(\mathcal{M})$ is called the Kapranov $L_{\infty}[1]$ algebra of the $\operatorname{dg}$ manifold $(\mathcal{M}, Q)$.

4.2. Recursive formula for multibrackets. It is clear that the Kapranov $L_{\infty}[1]$ algebra of a dg manifold in Theorem 4.4 is completely determined by the Atiyah 1-cocycle and

$$
R_{k} \in \Gamma\left(S^{k}\left(T_{\mathcal{M}}^{\vee}\right) \otimes T_{\mathcal{M}}\right) \cong \Gamma\left(\operatorname{Hom}\left(S^{k}\left(T_{\mathcal{M}}\right), T_{\mathcal{M}}\right)\right)
$$

for $k \geq 3$.

Recall that, for the $L_{\infty}[1]$ algebra on the Dolbeault complex $\Omega^{0, \bullet}\left(T_{X}^{1,0}\right)$ associated to the Atiyah class of the tangent bundle $T_{X}$ of a Kähler manifold $X$, Kapranov showed that the multibrackets can be described explicitly by a very simple formula: (4). For a general complex manifold, it was proved in [31] that they can be computed recursively as well. It is thus natural to ask if one can describe the multibrackets in Theorem 4.4 explicitly.

In what follows, we will give a characterization of these multibrackets, or equivalently all terms $R_{k}, k \geq 2$, by showing that they are completely determined by the Atiyah cocycle $\operatorname{At}_{(\mathcal{M}, Q)}^{\nabla}$, the curvature $R^{\nabla}$, and their higher covariant derivatives, by a recursive formula.

We need to introduce some notations first.

By $\widetilde{d^{\nabla}} R_{n-1} \in \Gamma\left(S^{n}\left(T_{\mathcal{M}}^{\vee}\right) \otimes T_{\mathcal{M}}\right)$, we denote the symmetrized covariant derivative of $R_{n-1}$. That is, for any $\boldsymbol{X} \in \Gamma\left(S^{n}\left(T_{\mathcal{M}}\right)\right)$,

$$
\begin{aligned}
\left(\widetilde{d^{\nabla}} R_{n-1}\right)(\boldsymbol{X}) & =\sum_{k=1}^{n} \varepsilon_{k}\left(\nabla_{X_{k}} R_{n-1}\right)\left(\boldsymbol{X}^{\{k\}}\right) \\
& =\sum_{k=1}^{n} \varepsilon_{k}\left((-1)^{\left|X_{k}\right|} \nabla_{X_{k}}\left(R_{n-1}\left(\boldsymbol{X}^{\{k\}}\right)\right)-R_{n-1}\left(\nabla_{X_{k}} \boldsymbol{X}^{\{k\}}\right)\right) .
\end{aligned}
$$

Here $\varepsilon_{k}=(-1)^{\left|X_{k}\right|\left(\left|X_{1}\right|+\cdots+\left|X_{k-1}\right|\right)}$ is the Koszul sign.

Let $B^{\nabla}: \Gamma\left(T_{\mathcal{M}} \otimes S\left(T_{\mathcal{M}}\right)\right) \rightarrow \Gamma\left(S\left(T_{\mathcal{M}}\right)\right)$ be the map defined by

$$
B^{\nabla}(Y ; \boldsymbol{X})=\left(\mathrm{pbw}^{\nabla}\right)^{-1}\left(Y \cdot \mathrm{pbw}^{\nabla}(\boldsymbol{X})\right)-\nabla_{Y} \boldsymbol{X}
$$

$\forall Y \in \mathfrak{X}(\mathcal{M})$ and $\boldsymbol{X} \in \Gamma\left(S^{n}\left(T_{\mathcal{M}}\right)\right)$. The following can be verified directly.

Lemma 4.5. The map $B^{\nabla}$ is well defined and $\mathcal{R}$-linear. Hence $B^{\nabla}$ is indeed a bundle map

$$
B^{\nabla}: T_{\mathcal{M}} \otimes S\left(T_{\mathcal{M}}\right) \rightarrow S\left(T_{\mathcal{M}}\right)
$$

As we will see below, the map $B^{\nabla}$ is completely determined by the curvature $R^{\nabla}$ and its higher covariant derivatives.

Let

$$
\Gamma\left(\widehat{S}\left(T_{\mathcal{M}}^{\vee}\right)\right) \otimes_{\mathcal{R}} \Gamma\left(S\left(T_{\mathcal{M}}\right)\right) \stackrel{\langle-\mid-\rangle}{\longrightarrow} \mathcal{R}
$$

be the duality pairing defined by

$$
\left\langle\alpha_{1} \odot \cdots \odot \alpha_{q} \mid X_{1} \odot \cdots \odot X_{p}\right\rangle= \begin{cases}\sum_{\sigma \in S_{p}} \varepsilon\left\langle\alpha_{1} \mid X_{\sigma(1)}\right\rangle \cdot\left\langle\alpha_{2} \mid X_{\sigma(2)}\right\rangle \cdots\left\langle\alpha_{p} \mid X_{\sigma(p)}\right\rangle & \text { if } p=q \\ 0 & \text { if } p \neq q\end{cases}
$$

for all homogeneous elements $\alpha_{1}, \ldots, \alpha_{q} \in \Gamma\left(T_{\mathcal{M}}^{\vee}\right)$ and $X_{1}, \ldots, X_{p} \in \Gamma\left(T_{\mathcal{M}}\right)$. The symbol $\varepsilon=\varepsilon\left(\alpha_{1}, \alpha_{2}, \cdots, \alpha_{p}, X_{1}, X_{2}, \cdots, X_{p}\right)$ denotes the Koszul signs arising from the reordering of the homogeneous objects $\alpha_{1}, \alpha_{2}, \cdots, \alpha_{p}, X_{1}, X_{2}, \cdots, X_{p}$ in each term of the right hand side.

The following is an immediate consequence of the Fedosov construction of graded manifolds [32, Theorem 5.6 and Proposition 5.2]. A short description on this topic can be found in Appendix A.

\section{Lemma 4.6.}


(i) The bundle map $B^{\nabla}: T_{\mathcal{M}} \otimes S\left(T_{\mathcal{M}}\right) \rightarrow S\left(T_{\mathcal{M}}\right)$ in Lemma 4.5 is completely determined by the curvature $R^{\nabla}$ and its higher covariant derivatives. More precisely, given any $Y \in \mathfrak{X}(\mathcal{M})$, provided that $B^{\nabla}(Y ; \boldsymbol{Y})$ is known for all $\boldsymbol{Y} \in \Gamma\left(S^{\leq n-1}\left(T_{\mathcal{M}}\right)\right)$, one can compute $B^{\nabla}(Y ; \boldsymbol{X})$ for any $\boldsymbol{X} \in \Gamma\left(S^{n}\left(T_{\mathcal{M}}\right)\right)$.

(ii) Moreover, if $R^{\nabla}=0$, then $B^{\nabla}(Y ; \boldsymbol{X})=Y \odot \boldsymbol{X}$, for all $Y \in \mathfrak{X}(\mathcal{M})$ and $\boldsymbol{X} \in \Gamma\left(S\left(T_{\mathcal{M}}\right)\right)$.

Proof. (i). Let

Then by Equation (39),

$$
\nabla_{Y}^{\xi} \boldsymbol{X}=\left(\mathrm{pbw}^{\nabla}\right)^{-1}\left(Y \cdot \mathrm{pbw}^{\nabla}(\boldsymbol{X})\right)
$$

$$
B^{\nabla}(Y ; \boldsymbol{X})=\nabla_{Y}^{\xi} \boldsymbol{X}-\nabla_{Y} \boldsymbol{X}
$$

For the rest of the proof, we follow the notation from Appendix A, in particular, Theorem A.4. For all $\sigma \in \Gamma\left(\widehat{S}\left(T_{\mathcal{M}}^{\vee}\right)\right)$, we have

$$
\begin{aligned}
\left\langle\sigma \mid \nabla_{Y}^{\xi} \boldsymbol{X}-\nabla_{Y} \boldsymbol{X}\right\rangle & =(-1)^{|\sigma| \cdot|Y|}\left\langle\nabla_{Y} \sigma-\nabla_{Y}^{\xi} \sigma \mid \boldsymbol{X}\right\rangle \\
& =(-1)^{|\sigma| \cdot|Y|}\left\langle i_{Y}\left(d^{\nabla}-d^{\nabla^{\xi}}\right)(\sigma) \mid \boldsymbol{X}\right\rangle \\
& =(-1)^{|\sigma| \cdot|Y|}\left\langle i_{Y}\left(\delta-\widetilde{A^{\nabla}}\right)(\sigma) \mid \boldsymbol{X}\right\rangle \\
& =\langle\sigma \mid Y \odot \boldsymbol{X}\rangle-(-1)^{|\sigma| \cdot|Y|}\left\langle i_{Y} \widetilde{A^{\nabla}}(\sigma) \mid \boldsymbol{X}\right\rangle \\
& =\langle\sigma \mid Y \odot \boldsymbol{X}\rangle-\left\langle\sigma \mid\left(i_{Y} \widetilde{A^{\nabla}}\right)^{T} \boldsymbol{X}\right\rangle .
\end{aligned}
$$

Thus it follows that

$$
B^{\nabla}(Y ; \boldsymbol{X})=Y \odot \boldsymbol{X}-\left(i_{Y} \widetilde{A^{\nabla}}\right)^{T} \boldsymbol{X}
$$

The conclusion thus follows from Corollary A.7.

(ii) Moreover, if $R^{\nabla}=0$, then $A^{\nabla}=0$ by Equation (67), and hence we obtain

$$
B^{\nabla}(Y ; \boldsymbol{X})=Y \odot \boldsymbol{X} .
$$

\section{Theorem 4.7.}

(i) The sections $R_{n} \in \Gamma\left(S^{n}\left(T_{\mathcal{M}}^{\vee}\right) \otimes T_{\mathcal{M}}\right)$, with $n \geq 3$, are completely determined by the Atiyah 1-cocycle $\operatorname{At}_{(\mathcal{M}, Q)}^{\nabla}$, the curvature $R^{\nabla}$, and their higher covariant derivatives, through the recursive formula

$$
R_{n}=\frac{2}{n}\left(\bar{R}_{2} \star \mathrm{id}\right)+\frac{1}{n} \sum_{k=2}^{n-1}\left[\left(\widetilde{\widetilde{d}}_{R^{\prime}} \star \mathrm{id}\right)+(1-k)\left(\bar{R}_{k} \star \mathrm{id}\right)-B^{\nabla} \circ\left(\bar{R}_{k} \otimes \mathrm{id}\right) \circ \Delta\right] .
$$

(ii) In particular, if $R^{\nabla}=0$, then $R_{2}=-\mathrm{At}_{(\mathcal{M}, Q)}^{\nabla}$ and $R_{n}=\frac{1}{n} \widetilde{d^{\nabla}} R_{n-1}$ for all $n \geq 3$.

In terms of Sweedler's notation $\Delta \boldsymbol{X}=\boldsymbol{X}_{(1)} \otimes \boldsymbol{X}_{(2)}$, one can rewrite Equation (40) as follows:

$$
\begin{aligned}
& R_{n}(\boldsymbol{X})=\frac{1}{n} \sum_{k=2}^{n-1}\left[\left(\widetilde{d^{\nabla}} R_{k}\left(\boldsymbol{X}_{(1)}\right) \odot \boldsymbol{X}_{(2)}\right)+(1-k)\left(R_{k}\left(\boldsymbol{X}_{(1)}\right) \odot \boldsymbol{X}_{(2)}\right)-B^{\nabla}\left(R_{k}\left(\boldsymbol{X}_{(1)}\right) ; \boldsymbol{X}_{(2)}\right)\right] \\
& +\frac{2}{n}\left(R_{2}\left(\boldsymbol{X}_{(1)}\right) \odot \boldsymbol{X}_{(2)}\right) .
\end{aligned}
$$


Now we proceed to prove Theorem 4.7. For any $\boldsymbol{X} \in \Gamma\left(S^{n}\left(T_{\mathcal{M}}\right)\right)$, we can write

$$
\begin{array}{rlrl}
C^{\nabla}(\boldsymbol{X}) & =\operatorname{pbw}^{\nabla} \circ\left(\left(\mathrm{pbw}^{\nabla}\right)^{-1} \circ \mathcal{L}_{Q}^{\mathcal{D}} \circ \mathrm{pbw}^{\nabla}-\mathcal{L}_{Q}\right)(\boldsymbol{X}) & \\
& =\operatorname{pbw}^{\nabla}\left(\sum_{k=0}^{n}\left(\bar{R}_{k} \star \mathrm{id}\right)(\boldsymbol{X})\right) & & \text { by Equation }(35) \\
& =\sum_{k=2}^{n} \operatorname{pbw}^{\nabla} \circ\left(\bar{R}_{k} \star \mathrm{id}\right)(\boldsymbol{X}) & & \text { by Equations }(36) .
\end{array}
$$

In order to simplify the notation, we introduce a sequence of maps $B_{k}^{\nabla}: \Gamma\left(S\left(T_{\mathcal{M}}\right)\right) \rightarrow \Gamma\left(S\left(T_{\mathcal{M}}\right)\right)$, for $k \geq 2$, defined by

$$
B_{k}^{\nabla}(\boldsymbol{X})=B^{\nabla} \circ\left(\bar{R}_{k} \otimes \mathrm{id}\right) \circ \Delta(\boldsymbol{X}), \quad \forall \boldsymbol{X} \in \Gamma\left(S^{n}\left(T_{\mathcal{M}}\right)\right) .
$$

Explicitly, in terms of Sweedler's notation $\Delta \boldsymbol{X}=\boldsymbol{X}_{(1)} \otimes \boldsymbol{X}_{(2)}$, we write

$$
\begin{aligned}
B_{k}^{\nabla}(\boldsymbol{X}) & =B^{\nabla}\left(R_{k}\left(\boldsymbol{X}_{(1)}\right) ; \boldsymbol{X}_{(2)}\right) \\
& =\left(\mathrm{pbw}^{\nabla}\right)^{-1}\left(R_{k}\left(\boldsymbol{X}_{(1)}\right) \cdot \mathrm{pbw}^{\nabla}\left(\boldsymbol{X}_{(2)}\right)\right)-\nabla_{R_{k}\left(\boldsymbol{X}_{(1)}\right)} \boldsymbol{X}_{(2)} .
\end{aligned}
$$

From Lemma 4.5 , it follows that $B_{k}^{\nabla}$, with $k \geq 2$, is $\mathcal{R}$-linear. That is, $B_{k}^{\nabla}$, with $k \geq 2$, is indeed a bundle map $S\left(T_{\mathcal{M}}\right) \rightarrow S\left(T_{\mathcal{M}}\right)$.

Proof of Theorem 4.7. (i) First, we will prove the recursive formula (40).

Pick any element $\boldsymbol{X}=X_{1} \odot \cdots \odot X_{n}$ in $\Gamma\left(S^{n}\left(T_{\mathcal{M}}\right)\right)$. Again, for the sake of simplicity, we use Sweedler's notation $\Delta \boldsymbol{X}=\boldsymbol{X}_{(1)} \otimes \boldsymbol{X}_{(2)}$ and the Koszul sign $\varepsilon_{k}=(-1)^{\left|X_{k}\right|\left(\left|X_{1}\right|+\cdots+\left|X_{k-1}\right|\right)}$. For each $l$, by Equations (16) and (32), we have

$$
\begin{aligned}
( & -l+1) \mathrm{pbw}^{\nabla} \circ\left(\bar{R}_{l} \star \mathrm{id}\right)(\boldsymbol{X}) \\
= & (n-l+1) \mathrm{pbw}^{\nabla}\left(R_{l}\left(\boldsymbol{X}_{(1)}\right) \odot \boldsymbol{X}_{(2)}\right) \\
= & R_{l}\left(\boldsymbol{X}_{(1)}\right) \cdot \mathrm{pbw}^{\nabla}\left(\boldsymbol{X}_{(2)}\right)-\mathrm{pbw}^{\nabla}\left(\nabla_{R_{l}\left(\boldsymbol{X}_{(1)}\right)} \boldsymbol{X}_{(2)}\right) \\
& +\sum_{k=1}^{n} \varepsilon_{k}(-1)^{\left|X_{k}\right|}\left(X_{k} \cdot \mathrm{pbw}^{\nabla}\left(R_{l}\left(\boldsymbol{X}_{(1)}^{\{k\}}\right) \odot \boldsymbol{X}_{(2)}^{\{k\}}\right)-\mathrm{pbw}^{\nabla}\left(\nabla_{X_{k}}\left(R_{l}\left(\boldsymbol{X}_{(1)}^{\{k\}}\right) \odot \boldsymbol{X}_{(2)}^{\{k\}}\right)\right)\right) \\
= & R_{l}\left(\boldsymbol{X}_{(1)}\right) \cdot \mathrm{pbw}^{\nabla}\left(\boldsymbol{X}_{(2)}\right)-\mathrm{pbw}^{\nabla}\left(\nabla_{R_{l}\left(\boldsymbol{X}_{(1)}\right)} \boldsymbol{X}_{(2)}\right) \\
& +\sum_{k=1}^{n} \varepsilon_{k}(-1)^{\left|X_{k}\right|}\left[X_{k} \cdot \mathrm{pbw}^{\nabla} \circ\left(\bar{R}_{l} \star \mathrm{id}\right)\left(\boldsymbol{X}^{\{k\}}\right)-\mathrm{pbw}^{\nabla}\left(\nabla_{X_{k}}\left(\left(\bar{R}_{l} \star \mathrm{id}\right)\left(\boldsymbol{X}^{\{k\}}\right)\right)\right)\right] .
\end{aligned}
$$

Combining it with Equation (42), we conclude that

$$
\begin{aligned}
& (n-l+1) \mathrm{pbw}^{\nabla} \circ\left(\bar{R}_{l} \star \mathrm{id}\right)(\boldsymbol{X})-\mathrm{pbw}^{\nabla} \circ B_{l}^{\nabla}(\boldsymbol{X}) \\
& =\sum_{k=1}^{n} \varepsilon_{k}(-1)^{\left|X_{k}\right|}\left[X_{k} \cdot \mathrm{pbw}^{\nabla} \circ\left(\bar{R}_{l} \star \mathrm{id}\right)\left(\boldsymbol{X}^{\{k\}}\right)-\mathrm{pbw}^{\nabla}\left(\nabla_{X_{k}}\left(\left(\bar{R}_{l} \star \mathrm{id}\right)\left(\boldsymbol{X}^{\{k\}}\right)\right)\right)\right] .
\end{aligned}
$$

Therefore,

$$
\begin{aligned}
(n-l & +1)\left(\bar{R}_{l} \star \mathrm{id}\right)(\boldsymbol{X})-B_{l}^{\nabla}(\boldsymbol{X}) \\
& =\sum_{k=1}^{n} \varepsilon_{k}(-1)^{\left|X_{k}\right|}\left[\left(\mathrm{pbw}^{\nabla}\right)^{-1}\left(X_{k} \cdot \mathrm{pbw}^{\nabla} \circ\left(\bar{R}_{l} \star \mathrm{id}\right)\left(\boldsymbol{X}^{\{k\}}\right)\right)-\nabla_{X_{k}}\left(\left(\bar{R}_{l} \star \mathrm{id}\right)\left(\boldsymbol{X}^{\{k\}}\right)\right)\right] .
\end{aligned}
$$


Also, for each $l$, by Equation (38), we have

$$
\begin{aligned}
& \left(\widetilde{d^{\nabla}} R_{l} \star \mathrm{id}\right)(\boldsymbol{X}) \\
= & \sum_{k=1}^{n} \varepsilon_{k}\left[\left(d^{\nabla} R_{l}\right)\left(X_{k} ; \boldsymbol{X}_{(1)}^{\{k\}}\right) \odot \boldsymbol{X}_{(2)}^{\{k\}}\right] \\
= & \sum_{k=1}^{n} \varepsilon_{k}\left[(-1)^{\left|X_{k}\right|}\left(\left(\nabla_{X_{k}} R_{l}\left(\boldsymbol{X}_{(1)}^{\{k\}}\right)\right) \odot \boldsymbol{X}_{(2)}^{\{k\}}\right)-\left(R_{l}\left(\nabla_{X_{k}} \boldsymbol{X}_{(1)}^{\{k\}}\right) \odot \boldsymbol{X}_{(2)}^{\{k\}}\right)\right] \\
= & \sum_{k=1}^{n} \varepsilon_{k}\left[(-1)^{\left|X_{k}\right|}\left(\left(\nabla_{X_{k}} R_{l}\left(\boldsymbol{X}_{(1)}^{\{k\}}\right)\right) \odot \boldsymbol{X}_{(2)}^{\{k\}}\right)+(-1)^{\left|X_{k}\right| \cdot\left|\boldsymbol{X}_{(1)}^{\{k\}}\right|}\left(R_{l}\left(\boldsymbol{X}_{(1)}^{\{k\}}\right) \odot\left(\nabla_{X_{k}} \boldsymbol{X}_{(2)}^{\{k\}}\right)\right)\right] \\
& -\sum_{k=1}^{n} \varepsilon_{k}\left[\left(R_{l}\left(\nabla_{X_{k}} \boldsymbol{X}_{(1)}^{\{k\}}\right) \odot \boldsymbol{X}_{(2)}^{\{k\}}\right)+(-1)^{\left|X_{k}\right| \cdot\left|\boldsymbol{X}_{(1)}^{\{k\}}\right|}\left(R_{l}\left(\boldsymbol{X}_{(1)}^{\{k\}}\right) \odot\left(\nabla_{X_{k}} \boldsymbol{X}_{(2)}^{\{k\}}\right)\right)\right] \\
= & \sum_{k=1}^{n} \varepsilon_{k}\left[(-1)^{\left|X_{k}\right|} \nabla_{X_{k}}\left(\left(\bar{R}_{l} \star \mathrm{id}\right)\left(\boldsymbol{X}^{\{k\}}\right)\right)-\left(\bar{R}_{l} \star \mathrm{id}\right)\left(\nabla_{X_{k}} \boldsymbol{X}^{\{k\}}\right)\right] .
\end{aligned}
$$

According to (36), we have $R_{2}=-\operatorname{At}_{(\mathcal{M}, Q)}^{\nabla}$. Hence

$$
\operatorname{pbw}^{\nabla} \circ\left(\bar{R}_{2} \star \mathrm{id}\right)(\boldsymbol{X})=-\sum_{i<j} \varepsilon_{i} \varepsilon_{j}(-1)^{\left|X_{i}\right| \cdot\left|X_{j}\right|} \mathrm{pbw}^{\nabla}\left(\operatorname{At}_{(\mathcal{M}, Q)}^{\nabla}\left(X_{i}, X_{j}\right) \odot \boldsymbol{X}^{\{i, j\}}\right) .
$$

By Equations (21) and (44), we have

$$
\begin{aligned}
& C^{\nabla}(\boldsymbol{X})-\frac{2}{n} \mathrm{pbw}^{\nabla} \circ\left(\bar{R}_{2} \star \mathrm{id}\right)(\boldsymbol{X}) \\
& =\frac{1}{n} \sum_{k=1}^{n} \varepsilon_{k}\left[(-1)^{\left|X_{k}\right|} X_{k} \cdot C^{\nabla}\left(\boldsymbol{X}^{\{k\}}\right)-C^{\nabla}\left(\nabla_{X_{k}} \boldsymbol{X}^{\{k\}}\right)\right] \\
& =\frac{1}{n} \sum_{k=1}^{n} \sum_{l=2}^{n-1} \varepsilon_{k}\left[(-1)^{\left|X_{k}\right|} X_{k} \cdot \mathrm{pbw}^{\nabla} \circ\left(\bar{R}_{l} \star \mathrm{id}\right)\left(\boldsymbol{X}^{\{k\}}\right)-\mathrm{pbw}^{\nabla} \circ\left(\bar{R}_{l} \star \mathrm{id}\right)\left(\nabla_{X_{k}} \boldsymbol{X}^{\{k\}}\right)\right] \\
& =\frac{1}{n} \sum_{k=1}^{n} \sum_{l=2}^{n-1} \varepsilon_{k}(-1)^{\left|X_{k}\right|}\left[X_{k} \cdot \mathrm{pbw}^{\nabla} \circ\left(\bar{R}_{l} \star \mathrm{id}\right)\left(\boldsymbol{X}^{\{k\}}\right)-\mathrm{pbw}^{\nabla}\left(\nabla_{X_{k}}\left(\left(\bar{R}_{l} \star \mathrm{id}\right)\left(\boldsymbol{X}^{\{k\}}\right)\right)\right)\right] \\
& \quad+\frac{1}{n} \sum_{k=1}^{n} \sum_{l=2}^{n-1} \varepsilon_{k}\left[(-1)^{\left|X_{k}\right|} \mathrm{pbw}^{\nabla}\left(\nabla_{X_{k}}\left(\left(\bar{R}_{l} \star \mathrm{id}\right)\left(\boldsymbol{X}^{\{k\}}\right)\right)\right)-\mathrm{pbw}^{\nabla} \circ\left(\bar{R}_{l} \star \mathrm{id}\right)\left(\nabla_{X_{k}} \boldsymbol{X}^{\{k\}}\right)\right],
\end{aligned}
$$

where the second equality is obtained by applying Equation (41) to $C^{\nabla}\left(\boldsymbol{X}^{\{k\}}\right)$ and $C^{\nabla}\left(\nabla_{X_{k}} \boldsymbol{X}^{\{k\}}\right)$.

It thus follows that

where

$$
\left(\mathrm{pbw}^{\nabla}\right)^{-1} \circ C^{\nabla}(\boldsymbol{X})-\frac{2}{n}\left(\bar{R}_{2} \star \mathrm{id}\right)(\boldsymbol{X})=\alpha+\beta,
$$

$$
\alpha=\frac{1}{n} \sum_{k=1}^{n} \sum_{l=2}^{n-1} \varepsilon_{k}(-1)^{\left|X_{k}\right|}\left[\left(\mathrm{pbw}^{\nabla}\right)^{-1}\left(X_{k} \cdot \mathrm{pbw}^{\nabla} \circ\left(\bar{R}_{l} \star \mathrm{id}\right)\left(\boldsymbol{X}^{\{k\}}\right)\right)-\nabla_{X_{k}}\left(\left(\bar{R}_{l} \star \mathrm{id}\right)\left(\boldsymbol{X}^{\{k\}}\right)\right)\right],
$$


and

$$
\begin{aligned}
& \beta=\frac{1}{n} \sum_{k=1}^{n} \sum_{l=2}^{n-1} \varepsilon_{k}\left[(-1)^{\left|X_{k}\right|}\left(\nabla_{X_{k}}\left(\left(\bar{R}_{l} \star \mathrm{id}\right)\left(\boldsymbol{X}^{\{k\}}\right)\right)\right)-\left(\bar{R}_{l} \star \mathrm{id}\right)\left(\nabla_{X_{k}} \boldsymbol{X}^{\{k\}}\right)\right] \\
& =\sum_{l=2}^{n-1} \frac{1}{n}\left(\widetilde{\widetilde{d^{\nabla}} R_{l}} \star \mathrm{id}\right)(\boldsymbol{X}) .
\end{aligned}
$$

Now, according to (43),

$$
\begin{aligned}
\alpha-\sum_{l=2}^{n-1}\left(\bar{R}_{l} \star \mathrm{id}\right)(\boldsymbol{X}) & =\sum_{l=2}^{n-1} \frac{1}{n}\left((n-l+1)\left(\bar{R}_{l} \star \mathrm{id}\right)(\boldsymbol{X})-B_{l}^{\nabla}(\boldsymbol{X})\right)-\sum_{l=2}^{n-1}\left(\bar{R}_{l} \star \mathrm{id}\right)(\boldsymbol{X}) \\
& =\frac{1}{n} \sum_{l=2}^{n-1}\left[(1-l)\left(\left(\bar{R}_{l} \star \mathrm{id}\right)(\boldsymbol{X})\right)-B_{l}^{\nabla}(\boldsymbol{X})\right] .
\end{aligned}
$$

Equation (41) can be rewritten as

$$
R_{n}(\boldsymbol{X})=\left(\mathrm{pbw}^{\nabla}\right)^{-1} \circ C^{\nabla}(\boldsymbol{X})-\sum_{k=2}^{n-1}\left(\bar{R}_{k} \star \mathrm{id}\right)(\boldsymbol{X}) .
$$

Equations (45), (46) and (47) then yield Equation (40).

From (36), we know that $R_{2}=-\operatorname{At}_{(\mathcal{M}, Q)}^{\nabla}$. According to Lemma 4.6, the bundle map $B^{\nabla}$ is completely determined by the curvature $R^{\nabla}$ and its higher covariant derivatives. It thus follows from the recursive formula (40) that, for any $n \geq 3, R_{n}$ is determined by $R_{k}$ with $k \leq n-1$, their covariant derivatives and the curvature. Thus, by inductive argument, $R_{n}$ is completely determined by the Atiyah 1-cocycle, the curvature and their higher covariant derivatives.

(ii) Assume that $R^{\nabla}=0$. By Lemma 4.6, the bundle map $B^{\nabla}: T_{\mathcal{M}} \otimes S\left(T_{\mathcal{M}}\right) \rightarrow S\left(T_{\mathcal{M}}\right)$ is given by $B^{\nabla}(Y ; \boldsymbol{X})=Y \odot \boldsymbol{X}$. Thus the formula $R_{n}(\boldsymbol{X})=\frac{1}{n} \widetilde{d^{\nabla}} R_{n-1}(\boldsymbol{X})$ can be obtained by induction argument, again using the recursive formula (40).

This concludes the proof of the theorem.

\section{EXAMPLES}

This section is devoted to the study of examples of Kapranov $L_{\infty}[1]$ algebras of some standard dg manifolds including those corresponding to $L_{\infty}[1]$ algebras, foliations and complex manifolds as in Examples 2.2 and 2.3.

5.1. dg manifolds associated to $L_{\infty}[1]$ algebras. Let $\mathfrak{g}$ be a finite dimensional $L_{\infty}$ algebra with $d=\operatorname{dim} \mathfrak{g}$. Then $\mathfrak{g}[1]$ is an $L_{\infty}[1]$ algebra: the (canonical) symmetric coalgebra $(S(\mathfrak{g}[1]), \Delta)$ is equipped with a coderivation $\widetilde{Q} \in \operatorname{coDer}(S(\mathfrak{g}[1]))$ of degree +1 satisfying $\widetilde{Q} \circ \widetilde{Q}=0$ and $\widetilde{Q}(1)=0$. Indeed, $\widetilde{Q}$ is equivalent to a sequence of linear maps $q_{k}: S^{k}(\mathfrak{g}[1]) \rightarrow \mathfrak{g}[1], k \geq 1$, of degree +1 satisfying the generalized Jacobi identities. The map $q_{k}$ is called the $k$-th multibracket.

Given an $L_{\infty}[1]$ algebra $\mathfrak{g}[1]$, we say a vector space $\mathfrak{M}$ is a $\mathfrak{g}[1]$-module if there exists a sequence of maps $\rho_{k}: S^{k}(\mathfrak{g}[1]) \otimes \mathfrak{M} \rightarrow \mathfrak{M}$ of degree $+1, \forall k \geq 0$, satisfying the standard compatibility condition [29]. If we write

$$
\rho=\sum_{k \geq 0} \rho_{k}: S(\mathfrak{g}[1]) \otimes \mathfrak{M} \rightarrow \mathfrak{M}
$$


the compatibility condition is expressed explicitly as

$$
\rho \circ\left(\left(\operatorname{id}_{S(\mathfrak{g}[1])} \otimes \rho\right) \circ\left(\Delta \otimes \operatorname{id}_{\mathfrak{M}}\right)+\widetilde{Q} \otimes \operatorname{id}_{\mathfrak{M}}\right)=0 .
$$

As an obvious example, we have the trivial module $\mathfrak{M}=\mathbb{K}$ together with the trivial action $\rho_{k}=0$ for all $k \geq 0$. Another example is the adjoint module $\mathfrak{M}=\mathfrak{g}[1]$ with the adjoint action $\rho_{k}: S^{k}(\mathfrak{g}[1]) \otimes \mathfrak{g}[1] \rightarrow \mathfrak{g}[1]$ defined by

$$
\rho_{k}(\boldsymbol{X} \otimes X)=q_{k+1}(\boldsymbol{X} \odot X),
$$

where $\boldsymbol{X} \in S^{k}(\mathfrak{g}[1]), X \in \mathfrak{g}[1]$ and $q_{k+1}: S^{k+1}(\mathfrak{g}[1]) \rightarrow \mathfrak{g}[1]$ is the multibracket of the $L_{\infty}$ [1] algebra $\mathfrak{g}[1]$. That is, $\left\{\rho_{k}\right\}_{k \geq 0}$ is defined by the following commutative diagram

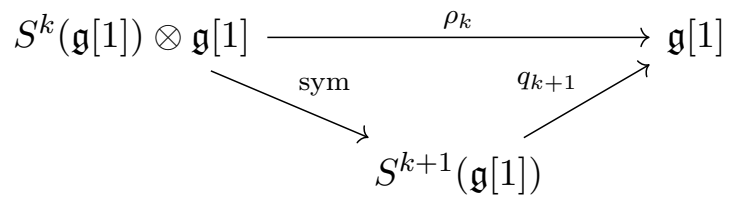

where $\operatorname{sym}: S^{\bullet}(\mathfrak{g}[1]) \otimes \mathfrak{g}[1] \rightarrow S^{\bullet+1}(\mathfrak{g}[1])$ is the canonical symmetrization map. By taking its dual, $(\mathfrak{g}[1])^{\vee}$ is also a $\mathfrak{g}[1]$-module, where the action is called the coadjoint action.

Throughout this section, we denote the degree of a homogeneous element $x \in \mathfrak{g}[1]$ by $|x|$. In particular, if $\mathfrak{g}$ is a Lie algebra concentrated in degree 0 , then for any $x \in \mathfrak{g}[1]$, its degree is $|x|=-1$.

The associated Chevalley-Eilenberg cochain complex of a $\mathfrak{g}[1]$-module $\mathfrak{M}$ is

$$
\mathcal{C}(\mathfrak{g}[1] ; \mathfrak{M})=\left(\operatorname{Hom}(S(\mathfrak{g}[1]), \mathfrak{M}), d_{\mathrm{CE}}^{\mathfrak{M}}\right),
$$

where $d_{\mathrm{CE}}^{\mathfrak{M}}$ is defined by

$$
d_{\mathrm{CE}}^{\mathfrak{M}}(F)=\rho \circ(\mathrm{id} \otimes F) \circ \Delta-(-1)^{|F|} F \circ \widetilde{Q},
$$

for any homogeneous element $F \in \operatorname{Hom}(S(\mathfrak{g}[1]), \mathfrak{M})$.

Observe that when $\mathfrak{M}$ is the trivial module $\mathbb{K}$, the associated Chevalley-Eilenberg cochain complex

$$
\mathcal{C}(\mathfrak{g}[1] ; \mathbb{K})=\left(\operatorname{Hom}(S(\mathfrak{g}[1]), \mathbb{K}), d_{\mathrm{CE}}^{\mathbb{K}}=d_{\mathrm{CE}}\right)
$$

is a dg algebra, equipped with the multiplication

$$
f \odot g=\mu_{\mathbb{K}} \circ(f \otimes g) \circ \Delta: S(\mathfrak{g}[1]) \rightarrow \mathbb{K}
$$

for any $f, g \in \operatorname{Hom}(S(\mathfrak{g}[1]), \mathbb{K})$. In other words, the $\operatorname{dg}$ algebra $\left(C^{\infty}(\mathfrak{g}[1]), Q\right)$ coincides with the Chevalley-Eilenberg cochain complex $\left(\mathcal{C}(\mathfrak{g}[1] ; \mathbb{K}), d_{\mathrm{CE}}\right)$ of the trivial $\mathfrak{g}[1]$-module $\mathbb{K}$. That is, $\left(\mathcal{C}(\mathfrak{g}[1] ; \mathbb{K}), d_{\mathrm{CE}}\right)$ is the dg algebra dual to the dg coalgebra $(S(\mathfrak{g}[1]), \widetilde{Q})$. Moreover, for any $\mathfrak{g}[1]$ module $\mathfrak{M}$, the Chevalley-Eilenberg cochain complex $\left(\mathcal{C}(\mathfrak{g}[1] ; \mathfrak{M}), d_{\mathrm{CE}}^{\mathfrak{M}}\right)$ is a dg module over the dg algebra $\left(C^{\infty}(\mathfrak{g}[1]), Q\right)$, where the action, under the identification $\mu_{0}: \mathbb{K} \otimes \mathfrak{M} \cong \mathfrak{M}$, is given by

$$
f \cdot F=\mu_{0} \circ(f \otimes F) \circ \Delta: S(\mathfrak{g}[1]) \rightarrow \mathfrak{M}
$$

for any $f \in \operatorname{Hom}(S(\mathfrak{g}[1]), \mathbb{K})$ and $F \in \operatorname{Hom}(S(\mathfrak{g}[1]), \mathfrak{M})$. In particular, this means that it satisfies the compatibility condition

$$
d_{\mathrm{CE}}^{\mathfrak{M}}(f \cdot F)=d_{\mathrm{CE}}(f) \cdot F+(-1)^{|f|} f \cdot d_{\mathrm{CE}}^{\mathfrak{M}}(F) .
$$

Therefore, the Chevalley-Eilenberg differential $d_{\mathrm{CE}}^{\mathfrak{M}}$ is completely determined by its image of elements in $\mathfrak{M}$, which is essentially induced by the action (48). More precisely, for any $x \in \mathfrak{M}$,

$$
d_{\mathrm{CE}}^{\mathfrak{M}}(x)=\sum_{k \geq 0} \rho_{k}(-, x) \in \operatorname{Hom}(S(\mathfrak{g}[1]), \mathfrak{M}) .
$$


In particular, if $\mathfrak{M}=\mathfrak{g}[1]$ is the adjoint module of the finite dimensional $L_{\infty}[1]$ algebra $\mathfrak{g}[1]$ described above, the Chevalley-Eilenberg differential $d_{\mathrm{CE}}^{\mathfrak{g}[1]}$ (seen as an operator on $\widehat{S}(\mathfrak{g}[1])^{\vee} \otimes \mathfrak{g}[1]$ ) is determined by the relation

$$
d_{\mathrm{CE}}^{\mathfrak{g}[1]}(x)=\sum_{k=1}^{\infty} \frac{1}{(k-1) !} \xi^{i_{k-1}} \odot \cdots \odot \xi^{i_{1}} \otimes q_{k}\left(e_{i_{1}} \odot \cdots \odot e_{i_{k-1}} \odot x\right), \quad \forall x \in \mathfrak{g}[1],
$$

where $\left\{e_{1}, \cdots, e_{d}\right\}$ is a basis for $\mathfrak{g}[1]$ and $\left\{\xi^{1}, \cdots, \xi^{d}\right\}$ is the dual basis for $(\mathfrak{g}[1])^{\vee}$. In Equation (52) and in the remainder of the present section, we use the Einstein notation tacitly to avoid inserting summations over the indices $i_{1}, \ldots, i_{k-1}$ in many equations.

Remark 5.1. In terms of Sweedler's notation, we may write (49) as

$$
(f \odot g)(\boldsymbol{X})=\sum_{(\boldsymbol{X})}(-1)^{|g| \cdot\left|\boldsymbol{X}_{(1)}\right|} f\left(\boldsymbol{X}_{(1)}\right) g\left(\boldsymbol{X}_{(2)}\right)
$$

and (50) as

$$
(f \cdot F)(\boldsymbol{X})=\sum_{(\boldsymbol{X})}(-1)^{|F| \cdot\left|\boldsymbol{X}_{(1)}\right|} f\left(\boldsymbol{X}_{(1)}\right) F\left(\boldsymbol{X}_{(2)}\right),
$$

where $f, g \in \operatorname{Hom}(S(\mathfrak{g}[1]), \mathbb{K}), F \in \operatorname{Hom}(S(\mathfrak{g}[1]), \mathfrak{M}), \boldsymbol{X} \in S(\mathfrak{g}[1])$ are homogeneous elements and $\Delta \boldsymbol{X}=\sum_{(\boldsymbol{X})} \boldsymbol{X}_{(1)} \otimes \boldsymbol{X}_{(2)}$

We now proceed to describe the Kapranov $L_{\infty}[1]$ algebra of the dg manifold $\left(\mathfrak{g}[1], d_{\mathrm{CE}}\right)$. Recall that $Q=d_{\mathrm{CE}}$ is defined by

$$
Q(f)=d_{\mathrm{CE}}(f)=-(-1)^{|f|} f \circ \widetilde{Q}
$$

for any homogeneous element $f \in \operatorname{Hom}(S(\mathfrak{g}[1]), \mathbb{K}) \cong C^{\infty}(\mathfrak{g}[1])$.

Let $\left\{e_{1}, \cdots, e_{d}\right\}$ be a basis of $\mathfrak{g}[1]$ and $\left\{x^{1}, \cdots, x^{d}\right\}$ its induced coordinate functions on $\mathfrak{g}[1]$ satisfying

$$
x^{i}\left(e_{j}\right)=\left\langle x^{i} \mid e_{j}\right\rangle=\left\{\begin{array}{ll}
1 & \text { if } i=j \\
0 & \text { if } i \neq j
\end{array} .\right.
$$

We also use the notation

$$
\frac{\partial}{\partial x^{j}} x^{i}:=(-1)^{\left|x^{i}\right| \cdot\left|x^{j}\right|}\left\langle x^{i} \mid e_{j}\right\rangle \text {. }
$$

Lemma 5.2. Under the above notation, write the multibrackets as

$$
q_{k}\left(e_{i_{1}}, \cdots, e_{i_{k}}\right)=\sum_{j} c_{i_{1} \cdots i_{k}}^{j} e_{j}, \quad \forall k \geq 1 .
$$

Then the homological vector field $Q \in \mathfrak{X}(\mathfrak{g}[1])$ can be written as

$$
Q=-\sum_{j} \sum_{k=1}^{\infty} \frac{1}{k !} c_{i_{1} \cdots i_{k}}^{j} x^{i_{k}} \odot \cdots \odot x^{i_{1}} \frac{\partial}{\partial x^{j}} .
$$

Here, we are making tacit use of the Einstein summation convention for the indices $i_{1}, \ldots, i_{k}$.

Proof. As a vector field, $Q$ can be written as $Q=\sum_{j} Q^{j} \frac{\partial}{\partial x^{j}}$ for some $Q^{j} \in C^{\infty}(\mathfrak{g}[1])$. Then, as a derivation of $C^{\infty}(\mathfrak{g}[1]), Q$ satisfies $Q\left(x^{j}\right)=(-1)^{\mid x^{j}} \mid Q^{j}$ according to (54). On the other hand, according to (53), we have

$$
\begin{aligned}
\left\langle Q\left(x^{j}\right) \mid e_{i_{1}} \odot \cdots \odot e_{i_{k}}\right\rangle & =-(-1)^{\left|x^{j}\right|}\left\langle x^{j} \mid \widetilde{Q}\left(e_{i_{1}} \odot \cdots \odot e_{i_{k}}\right)\right\rangle \\
& =-(-1)^{\left|x^{j}\right|} c_{i_{1} \cdots i_{k}}^{j}
\end{aligned}
$$

for any $k \geq 1$. 
Therefore, we may conclude that

$$
Q^{j}=-\sum_{k=1}^{\infty} \frac{1}{k !} c_{i_{1} \cdots i_{k}}^{j} x^{i_{k}} \odot \cdots \odot x^{i_{1}} .
$$

This completes the proof.

Note that we have a canonical trivialization of the tangent bundle

$$
T_{\mathfrak{g}[1]} \cong \mathfrak{g}[1] \times \mathfrak{g}[1] .
$$

Hence, we have the following identification

$$
\begin{aligned}
C^{\infty}(\mathfrak{g}[1]) \otimes \mathfrak{g}[1] & \leftarrow \mathfrak{X}(\mathfrak{g}[1]) \rightarrow \operatorname{Hom}(S(\mathfrak{g}[1]), \mathfrak{g}[1]) \\
f \otimes e_{i} & \leftarrow f \frac{\partial}{\partial x^{i}} \mapsto\left(\boldsymbol{X} \mapsto(-1)^{\left|e_{i}\right| \cdot|\boldsymbol{X}|}\langle f \mid \boldsymbol{X}\rangle \cdot e_{i}\right),
\end{aligned}
$$

where $f \in \operatorname{Hom}(S(\mathfrak{g}[1]), \mathbb{K}) \cong C^{\infty}(\mathfrak{g}[1])$ is homogeneous and $\boldsymbol{X} \in S(\mathfrak{g}[1])$.

Lemma 5.3. Under the identification (56), the Lie derivative $\mathcal{L}_{Q}=[Q,-] \in \operatorname{End}(\mathfrak{X}(\mathfrak{g}[1]))$ corresponds to the Chevalley-Eilenberg differential $d_{\mathrm{CE}}^{\mathfrak{g}[1]}$, where $\mathfrak{g}[1]$ acts on $\mathfrak{g}[1]$ by the adjoint action.

Proof. Recall that the Chevalley-Eilenberg differential $d_{\mathrm{CE}}^{\mathfrak{g}[1]}$ on $\mathfrak{g}[1]$ satisfies (51). On the other hand, we have

$$
\mathcal{L}_{Q}(f \cdot F)=[Q, f \cdot F]=Q(f) \cdot F+(-1)^{|f|} f \cdot[Q, F]=Q(f) \cdot F+(-1)^{|f|} f \cdot \mathcal{L}_{Q}(F),
$$

for any homogeneous element $f \in C^{\infty}(\mathfrak{g}[1]) \cong \operatorname{Hom}(S(\mathfrak{g}[1]), \mathbb{K})$ and $F \in \mathfrak{X}(\mathfrak{g}[1]) \cong \operatorname{Hom}(S(\mathfrak{g}[1]), \mathfrak{g}[1])$. Since $Q(f)=d_{\mathrm{CE}}(f)$ according to Equation (53), it suffices to prove the claim for each $\frac{\partial}{\partial x^{i}}$, $i=1, \ldots, d$.

We keep the notation $Q=\sum_{j} Q^{j} \frac{\partial}{\partial x^{j}}$. Now, by Lemma 5.2 , we have

$$
\begin{aligned}
\mathcal{L}_{Q}\left(\frac{\partial}{\partial x^{i}}\right) & =-(-1)^{\left|\frac{\partial}{\partial x^{i}}\right|} \sum_{j} \frac{\partial}{\partial x^{i}}\left(Q^{j}\right) \frac{\partial}{\partial x^{j}} \\
& =-(-1)^{\left|\frac{\partial}{\partial x^{i}}\right|}\left(-\sum_{j} \sum_{k=1}^{\infty} \frac{1}{k !} c_{i_{1} \cdots i_{k}}^{j} \frac{\partial\left(x^{i_{k}} \odot \cdots \odot x^{i_{1}}\right)}{\partial x^{i}} \frac{\partial}{\partial x^{j}}\right) \\
& =(-1)^{\left|\frac{\partial}{\partial x^{i}}\right|+\left|x^{i}\right|} \sum_{j} \sum_{k=1}^{\infty} \frac{1}{(k-1) !} c_{i_{1} \cdots i_{k-1} i}^{j} x^{i_{k-1}} \odot \cdots \odot x^{i_{1}} \frac{\partial}{\partial x^{j}} \\
& =\sum_{j} \sum_{k=1}^{\infty} \frac{1}{(k-1) !} c_{i_{1} \cdots i_{k-1} i}^{j} x^{i_{k-1}} \odot \cdots \odot x^{i_{1}} \frac{\partial}{\partial x^{j}} .
\end{aligned}
$$

The conclusion thus follows immediately by comparing the equation above with (52).

The trivialization of the tangent bundle (55) induces an isomorphism

$$
T_{\mathfrak{g}[1]}^{\vee} \otimes \operatorname{End}\left(T_{\mathfrak{g}[1]}\right) \cong \mathfrak{g}[1] \times\left((\mathfrak{g}[1])^{\vee} \otimes(\mathfrak{g}[1])^{\vee} \otimes \mathfrak{g}[1]\right)
$$

of vector bundles. Lemma 5.3, comparing with (6), indicates that we have an isomorphism of cochain complexes:

$$
\left(\Gamma\left(\mathfrak{g}[1] ; T_{\mathfrak{g}[1]}^{\vee} \otimes \operatorname{End}\left(T_{\mathfrak{g}[1]}\right)\right)^{\bullet}, \mathcal{Q}\right) \stackrel{\cong}{\rightarrow}\left(\operatorname{Hom}^{\bullet}(S(\mathfrak{g}[1]), \mathfrak{M}), d_{\mathrm{CE}}^{\mathfrak{M}}\right),
$$

where $\mathfrak{M}=(\mathfrak{g}[1])^{\vee} \otimes(\mathfrak{g}[1])^{\vee} \otimes \mathfrak{g}[1]$ is the tensor product of adjoint and coadjoint modules.

Thus we have the following 
Corollary 5.4. Let $(\mathcal{M}, Q)=\left(\mathfrak{g}[1], d_{\mathrm{CE}}\right)$ be the $d g$ manifold corresponding to a finite-dimensional $L_{\infty}[1]$ algebra $\mathfrak{g}[1]$. There is a canonical isomorphism, for any $k \in \mathbb{Z}$,

$$
H^{k}\left(\Gamma\left(T_{\mathfrak{g}[1]}^{\vee} \otimes \operatorname{End}\left(T_{\mathfrak{g}[1]}\right)\right)^{\bullet}, \mathcal{Q}\right) \cong H_{\mathrm{CE}}^{k}\left(\mathfrak{g}[1],(\mathfrak{g}[1])^{\vee} \otimes(\mathfrak{g}[1])^{\vee} \otimes \mathfrak{g}[1]\right)
$$

where the right hand side stands for the Chevalley-Eilenberg cohomology of the $L_{\infty}[1]$ algebra $\mathfrak{g}[1]$ with values in $(\mathfrak{g}[1])^{\vee} \otimes(\mathfrak{g}[1])^{\vee} \otimes \mathfrak{g}[1]$.

Remark 5.5. It is sometimes useful to use the Chevalley-Eilenberg cohomology of $L_{\infty}$ algebra rather than $L_{\infty}[1]$ algebra. Then Corollary 5.4 can be rephrased as follows.

For any finite-dimensional $L_{\infty}$ algebra $\mathfrak{g}$, there is a canonical isomorphism, for any $k \in \mathbb{Z}$,

$$
H^{k}\left(\Gamma\left(T_{\mathfrak{g}[1]}^{\vee} \otimes \operatorname{End}\left(T_{\mathfrak{g}[1]}\right)\right)^{\bullet}, \mathcal{Q}\right) \cong H_{\mathrm{CE}}^{k-1}\left(\mathfrak{g}, \mathfrak{g}^{\vee} \otimes \mathfrak{g}^{\vee} \otimes \mathfrak{g}\right)
$$

where the right hand side stands for the Chevalley-Eilenberg cohomology of the $L_{\infty}$ algebra $\mathfrak{g}$ with values in $\mathfrak{g}^{\vee} \otimes \mathfrak{g}^{\vee} \otimes \mathfrak{g}$. Note that there is a degree shifting here.

We still keep the notation $d_{\mathrm{CE}}=Q=\sum_{l} Q^{l} \frac{\partial}{\partial x^{l}}$. Let $\nabla: \mathfrak{X}(\mathfrak{g}[1]) \otimes \mathfrak{X}(\mathfrak{g}[1]) \rightarrow \mathfrak{X}(\mathfrak{g}[1])$ be the trivial (torsion-free) connection: $\nabla_{\frac{\partial}{\partial x^{i}}} \frac{\partial}{\partial x^{j}}=0$. The corresponding Atiyah 1-cocycle $\operatorname{At}_{\left(\mathfrak{g}[1], d_{\mathrm{CE}}\right)}^{\nabla} \in \Gamma\left(\operatorname{Hom}\left(S^{2}\left(T_{\mathfrak{g}[1]}\right), T_{\mathfrak{g}[1]}\right)\right)$ is completely determined by the relations

$$
\begin{aligned}
\operatorname{At}_{\left(\mathfrak{g}[1], d_{\mathrm{CE}}\right)}^{\nabla}\left(\frac{\partial}{\partial x^{i}}, \frac{\partial}{\partial x^{j}}\right) & =-(-1)^{\left|x^{i}\right|} \nabla_{\frac{\partial}{\partial x^{i}}} \mathcal{L}_{Q}\left(\frac{\partial}{\partial x^{j}}\right) \\
& =\sum_{l}(-1)^{\left|x^{i}\right|+\left|x^{j}\right|} \frac{\partial^{2}}{\partial x^{i} \partial x^{j}}\left(Q^{l}\right) \frac{\partial}{\partial x^{l}} \\
& =\sum_{l}(-1)^{\left|x^{i}\right|+\left|x^{j}\right|} \frac{\partial^{2}}{\partial x^{i} \partial x^{j}}\left(-\sum_{k=1}^{\infty} \frac{1}{k !} c_{i_{1} \cdots i_{k}}^{l} x^{i_{k}} \odot \cdots \odot x^{i_{1}}\right) \frac{\partial}{\partial x^{l}} \\
& =-\sum_{l} \sum_{k=2}^{\infty} \frac{1}{(k-2) !} c_{i_{1} \cdots i_{k-2} i j} x^{i_{k-2}} \odot \cdots \odot x^{i_{1}} \frac{\partial}{\partial x^{l}},
\end{aligned}
$$

for all $i, j \in\{1, \ldots, d\}$.

Let $\mathrm{At}_{\left(\mathfrak{g}[1], d_{\mathrm{CE}}\right)}$ be the map defined by the following commutative diagram

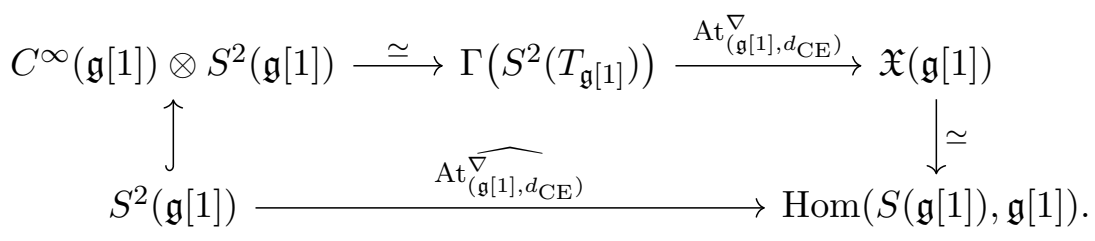

Equation (58) implies that

$$
\operatorname{At}_{\left(\mathfrak{g}[1], d_{\mathrm{CE}}\right)}\left(e_{i}, e_{j}\right): \quad e_{l_{1}} \odot \cdots \odot e_{l_{k}} \mapsto-q_{k+2}\left(e_{i} \odot e_{j} \odot e_{l_{1}} \odot \cdots \odot e_{l_{k}}\right) .
$$

Therefore, under the identification above, we have

$$
\operatorname{At}_{\left(\mathfrak{g}[1], d_{\mathrm{CE}}\right)}(x, y): \quad \boldsymbol{X} \mapsto-q_{n+2}(x \odot y \odot \boldsymbol{X}),
$$

for any $x, y \in \mathfrak{g}[1]$ and $\boldsymbol{X} \in S^{n}(\mathfrak{g}[1])$. Thus, by abuse of notation, we may write

$$
\operatorname{At}_{\left(\mathfrak{g}[1], d_{\mathrm{CE}}\right)}^{\nabla}=-\sum_{k \geq 2} q_{k}
$$


Proposition 5.6. Let $\mathfrak{g}[1]$ be an $L_{\infty}[1]$ algebra with multibrackets $q_{k}: S^{k}(\mathfrak{g}[1]) \rightarrow \mathfrak{g}[1], k \geq 1$. Then the Atiyah class $\alpha_{\left(\mathfrak{g}[1], d_{\mathrm{CE}}\right)}$ of the dg manifold $\left(\mathfrak{g}[1], d_{\mathrm{CE}}\right)$ is

$$
\alpha_{\left(\mathfrak{g}[1], d_{\mathrm{CE}}\right)}=-\left[\sum_{k \geq 2} q_{k}\right] \in H_{\mathrm{CE}}^{1}\left(\mathfrak{g}[1],(\mathfrak{g}[1])^{\vee} \otimes(\mathfrak{g}[1])^{\vee} \otimes \mathfrak{g}[1]\right) \cong H^{1}\left(\Gamma\left(T_{\mathfrak{g}[1]}^{\vee} \otimes \operatorname{End} T_{\mathfrak{g}[1]}\right)^{\bullet}, \mathcal{Q}\right) .
$$

Remark 5.7. We can rephrase Proposition 5.6 in terms of multibrackets of $L_{\infty}$ algebra $\mathfrak{g}$ instead of $L_{\infty}$ [1] algebra $\mathfrak{g}[1]$. For a finite dimensional $L_{\infty}$ algebra $\mathfrak{g}$ equipped with multibrackets $l_{k}: \Lambda^{k} \mathfrak{g} \rightarrow \mathfrak{g}$ of degree $2-k$ for $k \geq 1$, the Atiyah class $\alpha_{\left(\mathfrak{g}[1], d_{\mathrm{CE}}\right)}$ of the $\operatorname{dg}$ manifold $\left(\mathfrak{g}[1], d_{\mathrm{CE}}\right)$ is

$$
\alpha_{\left(\mathfrak{g}[1], d_{\mathrm{CE}}\right)}=\left[\sum_{k \geq 2} l_{k}\right] \in H_{\mathrm{CE}}^{0}\left(\mathfrak{g}, \mathfrak{g}^{\vee} \otimes \mathfrak{g}^{\vee} \otimes \mathfrak{g}\right) \cong H^{1}\left(\Gamma\left(T_{\mathfrak{g}[1]}^{\vee} \otimes \operatorname{End} T_{\mathfrak{g}[1]}\right), \mathcal{Q}\right),
$$

where $H_{\mathrm{CE}}^{0}\left(\mathfrak{g}, \mathfrak{g}^{\vee} \otimes \mathfrak{g}^{\vee} \otimes \mathfrak{g}\right)$ denotes the 0-th Chevalley-Eilenberg cohomology of the $L_{\infty}$ algebra $\mathfrak{g}$ with values in the tensor product of adjoint and coadjoint modules $\mathfrak{g}^{\vee} \otimes \mathfrak{g}^{\vee} \otimes \mathfrak{g}$.

Since the trivial connection $\nabla$ is flat, by the second part of Theorem 4.7 , we know that

$$
R_{n}=\frac{1}{n} \widetilde{d^{\nabla}} R_{n-1} \in \Gamma\left(\operatorname{Hom}\left(S^{n}\left(T_{\mathfrak{g}[1]}\right), T_{\mathfrak{g}[1]}\right)\right)
$$

for $n \geq 3$. As the connection $\nabla$ is trivial, Equation (38) implies that

$$
\begin{aligned}
\widetilde{d^{\nabla}} R_{n-1}\left(\frac{\partial}{\partial x^{i_{1}}} \odot \cdots \odot \frac{\partial}{\partial x^{i_{n}}}\right) & =\sum_{k=1}^{n} \varepsilon_{k}(-1)^{\left|x^{i_{k}}\right|} \nabla_{\frac{\partial}{\partial x^{i_{k}}}}\left(R_{n-1}\left(\frac{\partial}{\partial x^{i_{1}}} \odot \cdots \frac{\widehat{\partial}}{\partial x^{i_{k}}} \cdots \odot \frac{\partial}{\partial x^{i_{n}}}\right)\right) \\
& =\sum_{k=1}^{n} \varepsilon_{k}(-1)^{\left|x^{i_{k}}\right|} \frac{\partial}{\partial x^{i_{k}}}\left(R_{n-1}\left(\frac{\partial}{\partial x^{i_{1}}} \odot \cdots \frac{\widehat{\partial}}{\partial x^{i_{k}}} \cdots \odot \frac{\partial}{\partial x^{i_{n}}}\right)\right)
\end{aligned}
$$

Here, $\epsilon_{k}=(-1)^{\left|x^{i_{k}}\right|\left(\left|x^{i_{1}}\right| \cdots+\left|x^{i_{k-1}}\right|\right)}$ is the Koszul sign. Starting from

$$
R_{2}\left(\frac{\partial}{\partial x^{i_{1}}} \odot \frac{\partial}{\partial x^{i_{2}}}\right)=-(-1)^{\left|x^{i_{1}}\right|+\left|x^{i_{2}}\right|} \sum_{j} \frac{\partial^{2} Q^{j}}{\partial x^{i_{1}} \partial x^{i_{2}}} \frac{\partial}{\partial x^{j}},
$$

as in (57), we inductively obtain that

$$
R_{n}\left(\frac{\partial}{\partial x^{i_{1}}} \odot \cdots \odot \frac{\partial}{\partial x^{i_{n}}}\right)=-(-1)^{\left|x^{i_{1}}\right|+\cdots+\left|x^{i_{n}}\right|} \sum_{j} \frac{\partial^{n} Q^{j}}{\partial x^{i_{1}} \cdots \partial x^{i_{n}}} \frac{\partial}{\partial x^{j}} .
$$

According to Corollary 4.4, we obtain the following

Proposition 5.8. Let $\mathfrak{g}[1]$ be a finite dimensional $L_{\infty}[1]$ algebra with multibrackets $q_{k}: S^{k}(\mathfrak{g}[1]) \rightarrow \mathfrak{g}[1]$, $k \geq 1$. Let $(\mathcal{M}, Q)=\left(\mathfrak{g}[1], d_{\mathrm{CE}}\right)$ be its corresponding $d g$ manifold. Choose the trivial connection. Then the multibrackets $\left\{\lambda_{n}\right\}_{n \geq 1}$ of the Kapranov $L_{\infty}[1]$ algebra structure on $\operatorname{Hom}(S(\mathfrak{g}[1]), \mathfrak{g}[1]) \cong \widehat{S}(\mathfrak{g}[1])^{\vee} \otimes \mathfrak{g}[1]$, being identified with $\mathfrak{X}(\mathfrak{g}[1])$ as in Equation (56), are given as follows.

(1) The unary bracket $\lambda_{1}$ coincides with the Chevalley-Eilenberg differential with values in the $L_{\infty}[1]$-adjoint module $\mathfrak{g}[1]$ :

$$
\lambda_{1}=d_{\mathrm{CE}}^{\mathfrak{g}[1]}: \widehat{S}(\mathfrak{g}[1])^{\vee} \otimes \mathfrak{g}[1] \rightarrow \widehat{S}(\mathfrak{g}[1])^{\vee} \otimes \mathfrak{g}[1]
$$

(2) For any $n \geq 2, \lambda_{n}$ is $\widehat{S}(\mathfrak{g}[1])^{\vee}$-linear in each of its $n$ argument, and therefore can be considered as a linear map

$$
\lambda_{n}: S^{n}(\mathfrak{g}[1]) \rightarrow \widehat{S}(\mathfrak{g}[1])^{\vee} \otimes \mathfrak{g}[1]
$$


which is completely determined by

$$
\lambda_{n}(\boldsymbol{X})=\sum_{k=n}^{\infty} q_{k}(\boldsymbol{X} \odot-), \quad n \geq 2,
$$

where $\boldsymbol{X} \in S^{n}(\mathfrak{g}[1])$, and each $q_{k}(\boldsymbol{X} \odot-): S^{k-n}(\mathfrak{g}[1]) \rightarrow \mathfrak{g}[1]$ is defined by $\boldsymbol{Y} \mapsto q_{k}(\boldsymbol{X} \odot \boldsymbol{Y})$ for all $\boldsymbol{Y} \in S^{k-n}(\mathfrak{g}[1])$.

Example 5.9. If $\mathfrak{g}$ is a finite dimensional Lie algebra, then the Kapranov $L_{\infty}$ algebra (i.e. (-1)shifted Kapranov $L_{\infty}[1]$ algebra) of the dg manifold $\left(\mathfrak{g}[1], d_{\mathrm{CE}}\right)$ is the dgla $\Lambda \mathfrak{g}^{\vee} \otimes \mathfrak{g}$, where the differential is the Chevalley-Eilenberg differential $d_{\mathrm{CE}}^{\mathfrak{g}}$ of the $\mathfrak{g}$-module $\mathfrak{g}$ (for the adjoint action), and the Lie bracket is $[\xi \otimes x, \eta \otimes y]=\xi \wedge \eta \otimes[x, y]$ for homogeneous $\xi, \eta \in \Lambda \mathfrak{g}^{\vee}$ and $x, y \in \mathfrak{g}$.

5.2. dg manifolds associated to complex manifolds and integrable distributions. Every complex manifold $X$ determines a dg manifold $\left(T_{X}^{0,1}[1], \bar{\partial}\right)$ — see Example 2.3. This section is devoted to the description of the corresponding Kapranov $L_{\infty}[1]$ algebra. Recall that for a Kähler manifold $X$, Kapranov obtained an explicit description of an $L_{\infty}[1]$ algebra structure on the Dolbeault complex $\Omega^{0, \bullet}\left(T_{X}^{1,0}\right)$, where the unary bracket is the Dolbeault operator $\bar{\partial}$ and the binary bracket is the Dolbeault cocycle of the Atiyah class of $T_{X}$ [25, Theorem 2.6]. Kapranov proved the existence of an $L_{\infty}$ [1] algebra structure associated to the Atiyah class of the holomorphic tangent bundle of any complex manifold using formal geometry and PROP [25, Theorem 4.3]. See Theorem 5.11 below for the Dolbeault representations. Since $T_{X}^{0,1} \subset T_{\mathbb{C}} X$ is a complex integrable distribution, we will consider general integrable distributions over $\mathbb{K}$. Indeed such $L_{\infty}$ [1] algebra structures can be obtained in a more general perspective in terms of Lie pairs [31]. We recall its construction briefly below.

Let $F \subseteq T_{\mathbb{K}} M$ be an integrable distribution. Then $\left(F[1], d_{F}\right)$ is a dg manifold, whose algebra of smooth functions $C^{\infty}(F[1], \mathbb{K})$ is identified with $\Omega_{F}:=\Gamma\left(\wedge F^{\vee}\right)$ and the homological vector field is the leafwise de Rham differential, i.e. the Chevalley-Eilenberg differential $d_{F}: \Omega_{F}^{\bullet} \rightarrow \Omega_{F}^{\bullet+1}$ of the Lie algebroid $F$. It is well known that the normal bundle $B:=T_{\mathbb{K}} M / F$ is naturally an $F$-module, where the $F$-action is known as the Bott connection [14], defined by

$$
\nabla_{a}^{\mathrm{Bott}} b=q([a, \tilde{b}]),
$$

for all $a \in \Gamma(F), b \in \Gamma(B)$ and $\tilde{b} \in \Gamma\left(T_{\mathbb{K}} M\right)$ such that $q(\tilde{b})=b$. Here $q: T_{\mathbb{K}} M \rightarrow B$ denotes the canonical projection. Let $\mathcal{D}(M)$ be the space of $\mathbb{K}$-linear differential operators on $M$, and $R=C^{\infty}(M ; \mathbb{K})$ be the space of $\mathbb{K}$-valued smooth functions on $M$. Then $\mathcal{D}(M)$ is an $R$-coalgebra equipped with the standard coproduct

$$
\Delta: \mathcal{D}(M) \rightarrow \mathcal{D}(M) \otimes_{R} \mathcal{D}(M)
$$

Let $\mathcal{D}(M) \Gamma(F) \subseteq \mathcal{D}(M)$ be the left ideal of $\mathcal{D}(M)$ generated by $\Gamma(F)$. Since

$$
\Delta(\mathcal{D}(M) \Gamma(F)) \subseteq \mathcal{D}(M) \otimes_{R} \mathcal{D}(M) \Gamma(F)+\mathcal{D}(M) \Gamma(F) \otimes_{R} \mathcal{D}(M),
$$

the coproduct (59) descends to a well-defined coproduct over $R$

$$
\Delta: \mathcal{D}(B) \rightarrow \mathcal{D}(B) \otimes_{R} \mathcal{D}(B),
$$

on the quotient space $\mathcal{D}(B):=\frac{\mathcal{D}(M)}{\mathcal{D}(M) \Gamma(F)}$. Hence $\mathcal{D}(B)$ is an $R$-coalgebra as well, called the $R$ coalgebra of differential operators transverse to $F[52]$.

It is well known that $\mathcal{D}(B)$ is an $F$-module $[31,30]$, where the $F$-action is given by

$$
a \cdot \bar{u}=\overline{a \circ u},
$$

for any $a \in \Gamma(F)$ and $u \in \mathcal{D}(M)$ - the symbol $\bar{x}$ denotes the image of $x$ under the quotient map $\mathcal{D}(M) \rightarrow \mathcal{D}(B)$. Here $\circ$ denotes the composition of differential operators. Moreover, $F$ acts on 
$\mathcal{D}(B)$ by coderivations. Indeed, the associated Chevalley-Eilenberg differential

$$
d_{F}^{\mathcal{U}}: \quad \Omega_{F}^{\bullet}(\mathcal{D}(B)) \rightarrow \Omega_{F}^{\bullet+1}(\mathcal{D}(B))
$$

is a coderivation of the $\Omega_{F}$-linear coproduct

$$
\Delta: \Omega_{F}(\mathcal{D}(B)) \rightarrow \Omega_{F}(\mathcal{D}(B)) \otimes_{\Omega_{F}} \Omega_{F}(\mathcal{D}(B))
$$

extending the coproduct $(60)$ on $\mathcal{D}(B)$. Thus $\left(\Omega_{F}(\mathcal{D}(B)), d_{F}^{\mathcal{U}}, \Delta\right)$ is a dg coalgebra over $\left(\Omega_{F}, d_{F}\right)$.

Let $j: B \rightarrow T_{\mathbb{K}} M$ be a splitting of the short exact sequence of vector bundles over $M$ :

$$
0 \rightarrow F \stackrel{i}{\rightarrow} T_{\mathbb{K}} M \stackrel{q}{\rightarrow} B \rightarrow 0 .
$$

Choose a torsion-free linear connection $\nabla^{B}$ of the vector bundle $B$, i.e. a $T_{\mathbb{K}} M$-connection on $B$ satisfying the condition:

$$
\nabla_{X}^{B}(q(Y))-\nabla_{Y}^{B}(q(X))-q([X, Y])=0
$$

for any $X, Y \in \Gamma\left(T_{\mathbb{K}} M\right)$. It is known [31, Lemma 5.2] that a torsion-free linear connection $\nabla^{B}$ automatically extends the Bott representation of $F$ on $B$, that is, $\nabla_{a}^{B} \bar{X}=\nabla_{a}^{\text {Bott }} \bar{X}, \forall a \in \Gamma(F)$ and $\bar{X} \in \Gamma(B)$.

According to [31, 30], the pair $\left(j, \nabla^{B}\right)$ determines an isomorphism of $R$-coalgebras

$$
\overline{\mathrm{pbw}}: \Gamma(S(B)) \rightarrow \mathcal{D}(B),
$$

called the PBW isomorphism for the Lie pair $\left(T_{\mathbb{K}} M, F\right)$, which is defined recursively by the relations

$$
\begin{aligned}
& \overline{\mathrm{pbw}}(f)=f, \quad \forall f \in R, \\
& \overline{\operatorname{pbw}}(b)=\overline{j(b)}, \quad \forall b \in \Gamma(B),
\end{aligned}
$$

and

$$
\overline{\operatorname{pbw}}\left(b_{1} \odot \cdots \odot b_{n}\right)=\frac{1}{n} \sum_{k=1}^{n}\left\{j\left(b_{k}\right) \cdot \overline{\operatorname{pbw}}\left(b^{\{k\}}\right)-\overline{\operatorname{pbw}}\left(\nabla_{j\left(b_{k}\right)}^{B}\left(b^{\{k\}}\right)\right)\right\},
$$

where we keep the notation from $(61)$ and $b^{\{k\}}=b_{1} \odot \cdots \odot b_{k-1} \odot b_{k+1} \odot \cdots \odot b_{n}$. Extending this isomorphism of $R$-coalgebras $\Omega_{F}$-linearly, we obtain an isomorphism of $\Omega_{F}$-coalgebras

$$
\overline{\mathrm{pbw}}: \Omega_{F}(S(B)) \rightarrow \Omega_{F}(\mathcal{D}(B)) .
$$

Transferring the coderivation $d_{F}^{\mathcal{U}}$ of $\Omega_{F}(\mathcal{D}(B))$ to $\Omega_{F}(S(B))$ via the isomorphism (63), we obtain a degree +1 coderivation $\bar{\delta}$ of $\Omega_{F}(S(B))$ :

$$
\bar{\delta}:=(\overline{\mathrm{pbw}})^{-1} \circ d_{F}^{\mathcal{U}} \circ \overline{\mathrm{pbw}}: \Omega_{F}^{\bullet}(S(B)) \rightarrow \Omega_{F}^{\bullet+1}(S(B)) .
$$

Thus

is a dg coalgebra over $\left(\Omega_{F}, d_{F}\right)$.

$$
\left(\Omega_{F}(S(B)), \bar{\delta}, \Delta\right)
$$

By dualizing $\bar{\delta}$ over the dg algebra $\left(\Omega_{F}, d_{F}\right)$, we obtain a degree +1 derivation

$$
D: \Omega_{F}^{\bullet}\left(\widehat{S}\left(B^{\vee}\right)\right) \rightarrow \Omega_{F}^{\bullet+1}\left(\widehat{S}\left(B^{\vee}\right)\right) .
$$

According to [31, Theorem 5.7], $D$ in (64) can be expressed as

$$
D=d_{F}^{\nabla^{\mathrm{Bott}}}+\sum_{k=2}^{\infty} \widetilde{\mathcal{R}}_{k},
$$

where

(1) $d_{F}^{\nabla^{\text {Bott }}}$ is the Chevalley-Eilenberg differential corresponding to the Bott connection of $F$ on $\widehat{S}\left(B^{\vee}\right)$; 
(2) for any $k \geq 2, \widetilde{\mathcal{R}}_{k}: \Omega_{F}^{\bullet}\left(\widehat{S}\left(B^{\vee}\right)\right) \rightarrow \Omega_{F}^{\bullet+1}\left(\widehat{S}\left(B^{\vee}\right)\right)$ is the $\Omega_{F}^{\bullet}$-linear degree +1 derivation acting by contraction induced from a section $\mathcal{R}_{k} \in \Omega_{F}^{1}\left(S^{k}\left(B^{\vee}\right) \otimes B\right)$;

(3) $\mathcal{R}_{2} \in \Omega_{F}^{1}\left(S^{2}\left(B^{\vee}\right) \otimes B\right)$ is the Atiyah 1-cocycle $\mathrm{At}_{T_{\mathbb{K}} M / F}^{\nabla^{\text {Bott }}}$ associated with the connection $\nabla^{B}$ defined by

$$
\mathcal{R}_{2}(a, \bar{X})=\nabla_{a}^{B} \nabla_{X}^{B}-\nabla_{X}^{B} \nabla_{a}^{B}-\nabla_{[a, X]}^{B},
$$

for all $a \in \Gamma(F)$ and $X \in \Gamma\left(T_{\mathbb{K}} M\right)$, where $\bar{X} \in \Gamma(B)$ denotes the image of $X$ under the quotient $\operatorname{map} T_{\mathbb{K}} M \rightarrow T_{\mathbb{K}} M / F$.

A priori, $\mathcal{R}_{2} \in \Omega_{F}^{1}\left(B^{\vee} \otimes \operatorname{End}(B)\right)$, but the torsion-free assumption guarantees that it is indeed an element in $\Omega_{F}^{1}\left(S^{2}\left(B^{\vee}\right) \otimes B\right)$. Its cohomology class $\alpha_{T_{\mathbb{K}} M / F} \in \mathbb{H}_{\mathrm{CE}}^{1}\left(F, B^{\vee} \otimes \operatorname{End}(B)\right)$ is independent of the choice of $\nabla^{B}$ and is called the Atiyah class of the Lie pair $\left(T_{\mathbb{K}} M, F\right)$ [14]. Note that $\Omega_{F}\left(\widehat{S}\left(B^{\vee}\right)\right)$ is the algebra of functions on $F[1] \oplus B$. Thus $(F[1] \oplus B, D)$ is a dg manifold with support $M$, called a Kapranov $\boldsymbol{d g}$ manifold associated to the Lie pair $\left(T_{\mathbb{K}} M, F\right)$ [31]. One can prove that the various Kapranov dg manifold structures on $F[1] \oplus B$ resulting from all possible choices of splitting and connection are all isomorphic.

Theorem 5.10 ([31, Theorem 5.7]). Let $F \subseteq T_{\mathbb{K}} M$ be an integrable distribution. The choice of a splitting $j: B \rightarrow T_{\mathbb{K}} M$ of the short exact sequence (62) and a torsion-free linear connection $\nabla^{B}$ of the vector bundle $B$ determines an $L_{\infty}[1]$ algebra structure on the graded vector space $\Omega_{F}^{\bullet}(B)$ defined by a sequence $\left(\lambda_{k}\right)_{k \in \mathbb{N}}$ of multibrackets such that each $\lambda_{k}$, with $k \geqslant 2$, is $\Omega_{F-\text { multilinear, }}$ and

- the unary bracket $\lambda_{1}$ is the Chevalley-Eilenberg differential $d_{F}^{\nabla^{\text {Bott }}}$ associated with the Bott connection $\nabla^{\text {Bott }}$ of $F$ on $B$;

- the binary bracket $\lambda_{2}$ is the map

$$
\lambda_{2}: \Omega_{F}^{j_{1}}(B) \otimes \Omega_{F}^{j_{2}}(B) \rightarrow \Omega_{F}^{j_{1}+j_{2}+1}(B)
$$

induced by the Atiyah 1-cocycle $\mathcal{R}_{2} \in \Omega_{F}^{1}\left(S^{2}\left(B^{\vee}\right) \otimes B\right)$;

- for every $k \geqslant 3$, the $k$-th multibracket $\lambda_{k}$ is the composition of the wedge product

$$
\Omega_{F}^{j_{1}}(B) \otimes \cdots \otimes \Omega_{F}^{j_{k}}(B) \rightarrow \Omega_{F}^{j_{1}+\cdots+j_{k}}\left(B^{\otimes k}\right)
$$

with the map

$$
\Omega_{F}^{j_{1}+\cdots+j_{k}}\left(B^{\otimes k}\right) \rightarrow \Omega_{F}^{j_{1}+\cdots+j_{k}+1}(B)
$$

induced by an element $\left.R_{k} \in \Omega_{F}^{1}\left(S^{k}\left(B^{\vee}\right) \otimes B\right) \subset \Omega_{F}^{1}\left(\left(B^{\vee}\right)^{\otimes k} \otimes B\right)\right)$.

Moreover, the $L_{\infty}[1]$ algebra structure on $\Omega_{F}^{\bullet}(B)$ is unique up to isomorphisms in the sense that those resulting from all possible choices of splitting and connection are all isomorphic.

Any such $L_{\infty}[1]$ algebra structure on $\Omega_{F}^{\bullet}(B)$ is called a Kapranov $L_{\infty}[1]$ algebra of the integrable distribution $F$.

As a special case, consider a complex manifold $X$. The subbundle $F=T_{X}^{0,1} \subset T_{\mathbb{C}} X$ is an integrable distribution, and the normal bundle $B:=T_{\mathbb{C}} X / T_{X}^{0,1}$ is naturally identified with $T_{X}^{1,0}$. Moreover, the Chevalley-Eilenberg differential associated with the Bott $F$-connection on $T_{X}^{1,0}$ becomes the Dolbeault operator

$$
\bar{\partial}: \Omega_{X}^{0, \bullet}\left(T_{X}^{1,0}\right) \rightarrow \Omega_{X}^{0, \bullet+1}\left(T_{X}^{1,0}\right) .
$$

The following is an immediate consequence of Theorem 5.10, which extends Kapranov's construction for Kähler manifolds [25, Theorem 2.6] to all complex manifolds.

Theorem 5.11 ([31, Theorem 5.24]). For a given complex manifold $X$, any torsion-free $T_{X}^{1,0}$ connection $\nabla^{1,0}$ on $T_{X}^{1,0}$ determines an $L_{\infty}[1]$ algebra structure on the Dolbeault complex $\Omega^{0, \bullet}\left(T_{X}^{1,0}\right)$ such that 
- the unary bracket $\lambda_{1}$ is the Dolbeault operator

$$
\bar{\partial}: \Omega^{0, j}\left(T_{X}^{1,0}\right) \rightarrow \Omega^{0, j+1}\left(T_{X}^{1,0}\right) ;
$$

- the binary bracket $\lambda_{2}$ is the map

$$
\lambda_{2}: \Omega^{0, j_{1}}\left(T_{X}^{1,0}\right) \otimes \Omega^{0, j_{2}}\left(T_{X}^{1,0}\right) \rightarrow \Omega^{0, j_{1}+j_{2}+1}\left(T_{X}^{1,0}\right)
$$

induced by the Dolbeault representative of the Atiyah 1-cocycle $R_{2} \in \Omega^{0,1}\left(S^{2}\left(T_{X}^{1,0}\right)^{\vee} \otimes T_{X}^{1,0}\right)$;

- for every $k \geqslant 3$, the $k$-th multibracket $\lambda_{k}$ is the composition of the wedge product

$$
\Omega^{0, j_{1}}\left(T_{X}^{1,0}\right) \otimes \cdots \otimes \Omega^{0, j_{k}}\left(T_{X}^{1,0}\right) \rightarrow \Omega^{0, j_{1}+\cdots+j_{k}}\left(\left(T_{X}^{1,0}\right)^{\otimes k}\right)
$$

with the map

$$
\Omega^{0, j_{1}+\cdots+j_{k}}\left(\left(T_{X}^{1,0}\right)^{\otimes k}\right) \rightarrow \Omega^{0, j_{1}+\cdots+j_{k}+1}\left(T_{X}^{1,0}\right)
$$

induced by an element $R_{k}$ of $\Omega^{0,1}\left(S^{k}\left(\left(T_{X}^{1,0}\right)^{\vee}\right) \otimes T_{X}^{1,0}\right) \subset \Omega^{0,1}\left(\left(\left(T_{X}^{1,0}\right)^{\vee}\right)^{\otimes k} \otimes T_{X}^{1,0}\right)$, which is itself completely determined by the Atiyah 1-cocycle $R_{2}$, the curvature of $\nabla^{1,0}$, and their higher covariant derivatives.

Moreover, the $L_{\infty}[1]$ algebra structure on $\Omega^{0, \bullet}\left(T_{X}^{1,0}\right)$ is unique up to isomorphisms.

Now we are ready to consider the Kapranov $L_{\infty}[1]$ algebra of the dg manifold $\left(F[1], d_{F}\right)$. Let

$$
\tilde{\Phi}: \mathcal{D}(F[1]) \rightarrow \Omega_{F}(\mathcal{D}(B))
$$

be the map defined by $\tilde{\Phi}(D)=\overline{\pi_{*}(D)}$, where $\pi_{*}: \mathcal{D}(F[1]) \rightarrow \Omega_{F} \otimes_{R} \mathcal{D}(M)$ is the pushforward map

$$
\pi_{*}(D)(f)=D\left(\pi^{*} f\right), \quad \forall D \in \mathcal{D}(F[1]), \forall f \in R
$$

and $\overline{\pi_{*}(D)} \in \Omega_{F}(\mathcal{D}(B))$ denotes the class of $\pi_{*}(D)$ in $\Omega_{F} \otimes_{R} \frac{\mathcal{D}(M)}{\mathcal{D}(M) \Gamma(F)} \cong \Omega_{F}(\mathcal{D}(B))$.

Theorem $5.12([52,16])$. There exists a contraction of $d g \Omega_{F}$-modules

$$
\tilde{H} \bigodot\left(\mathcal{D}(F[1]), \mathcal{L}_{Q}^{\mathcal{D}}\right) \underset{\tilde{\Psi}}{\stackrel{\tilde{\Phi}}{\rightleftarrows}}\left(\Omega_{F}(\mathcal{D}(B)), d_{F}^{\mathcal{U}}\right),
$$

where the projection $\tilde{\Phi}$ is a morphism of $\Omega_{F}$-coalgebras.

Choose a torsion-free affine connection $\nabla$ on $F[1]$. We write

$$
\text { pbw : } \Gamma\left(S\left(T_{F[1]}\right)\right) \rightarrow \mathcal{D}(F[1])
$$

for the corresponding Poincaré-Birkhoff-Witt map as in (14).

By conjugating the PBW maps pbw and $\overline{\mathrm{pbw}}$, respectively, on the left hand side and on the right hand side of (65), we obtain

Corollary 5.13. There exists a contraction of $d g \Omega_{F}$-modules

$$
H \bigodot\left(\Gamma\left(S\left(T_{F[1]}\right)\right), \mathrm{pbw}^{-1} \circ \mathcal{L}_{Q}^{\mathcal{D}} \circ \mathrm{pbw}\right) \underset{\Psi}{\stackrel{\Phi}{\rightleftarrows}}\left(\Omega_{F}(S(B)), \overline{\mathrm{pbw}}^{-1} \circ d_{F}^{\mathcal{U}} \circ \overline{\mathrm{pbw}}\right),
$$

where the projection $\Phi:=\overline{\mathrm{pbw}}^{-1} \circ \tilde{\Phi} \circ \mathrm{pbw}$ is a morphism of $\Omega_{F}$-coalgebras.

The projection $\Phi$ determines a sequence of maps $\left\{\phi_{k}\right\}_{k \geq 1}$ making the diagrams

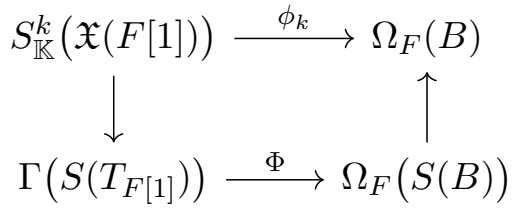


commutative. Note that $\phi_{1}: \mathfrak{X}(F[1]) \rightarrow \Omega_{F}(B)$ is the composition

$$
\mathfrak{X}(F[1]) \stackrel{\pi_{*}}{\rightarrow} \Omega_{F}\left(T_{\mathbb{K}} M\right) \stackrel{q}{\rightarrow} \Omega_{F}(B) .
$$

Theorem 5.14. Let $F \subseteq T_{\mathbb{K}} M$ be an integrable distribution. Then the sequence of $\Omega_{F}$-multilinear maps $\left\{\phi_{k}\right\}_{k \geq 1}$ defined by the commutative diagrams (66) constitutes a quasi-isomorphism from the Kapranov $L_{\infty}[1]$ algebra $\mathfrak{X}(F[1])$ arising from the dg manifold $\left(F[1], d_{F}\right)$ to the Kapranov $L_{\infty}[1]$ algebra $\Omega_{F}^{\bullet}(B)$ arising (as in Theorem 5.10) from the integrable distribution $F$.

As an immediate consequence, we have

Corollary 5.15. For any complex manifold $X$, consider its corresponding dg manifold $\left(T_{X}^{0,1}[1], \bar{\partial}\right)$ as in Example 2.3. The Kapranov $L_{\infty}[1]$ algebra $\mathfrak{X}\left(T_{X}^{0,1}[1]\right)$ is quasi-isomorphic to the $L_{\infty}$ [1] algebra $\Omega^{0, \bullet}\left(T_{X}^{1,0}\right)$-see Theorem 5.11. The quasi-isomorphism $\left\{\phi_{k}\right\}_{k \geq 1}$, in which each map $\phi_{k}$ is $\Omega_{X}^{0, \bullet}$ multilinear, is given by (66) (with $F=T_{X}^{0,1}$ and $B=T_{X}^{1,0}$ ), and in particular, the linear part $\phi_{1}: \mathfrak{X}\left(T_{X}^{0,1}[1]\right) \rightarrow \Omega^{0, \bullet}\left(T_{X}^{1,0}\right)$ is given by the composition

$$
\mathfrak{X}\left(T_{X}^{0,1}[1]\right) \stackrel{\pi_{*}}{\rightarrow} \Omega^{0, \bullet}\left(T_{X}^{\mathbb{C}}\right) \stackrel{\mathrm{pr}}{\longrightarrow} \Omega^{0, \bullet}\left(T_{X}^{1,0}\right) .
$$

\section{Appendix A. Fedosov COnStruction on Graded manifolds}

This section is to give a brief description of Fedosov construction of graded manifolds. We refer readers to $[18,19,32]$ for more details.

Throughout this section, $\mathcal{M}$ is a finite dimensional graded manifold and $\nabla$ is a torsion-free affine connection on $\mathcal{M}$. There is an induced linear connection on $\widehat{S}\left(T_{\mathcal{M}}^{\vee}\right)$, which is denoted by the same symbol $\nabla$ by abuse of notation.

Consider the map $\nabla^{\mathfrak{k}}: \mathfrak{X}(\mathcal{M}) \times \Gamma\left(S\left(T_{\mathcal{M}}\right)\right) \rightarrow \Gamma\left(S\left(T_{\mathcal{M}}\right)\right)$

$$
\nabla_{Y}^{\xi} \boldsymbol{X}=\left(\mathrm{pbw}^{\nabla}\right)^{-1}\left(Y \cdot \operatorname{pbw}^{\nabla}(\boldsymbol{X})\right)
$$

for any $Y \in \mathfrak{X}(\mathcal{M})$ and $\boldsymbol{X} \in \Gamma\left(S\left(T_{\mathcal{M}}\right)\right)$.

Lemma A.1. The above map $\nabla^{\text {主}}$ defines a flat connection on $S\left(T_{\mathcal{M}}\right)$.

Abusing notation, we write the same symbol $\nabla^{\xi}$ to denote the induced flat connection on $\widehat{S}\left(T_{\mathcal{M}}^{\vee}\right)$. Then the associated covariant derivative $d^{\nabla^{k}}$ satisfies $\left(d^{\nabla^{k}}\right)^{2}=0$.

In the following, we use the identification

$$
\Omega^{p}\left(\widehat{S}\left(T_{\mathcal{M}}^{\vee}\right)\right) \cong \Gamma\left(\Lambda^{p}\left(T_{\mathcal{M}}^{\vee}\right) \otimes \widehat{S}\left(T_{\mathcal{M}}^{\vee}\right)\right) \cong \Gamma\left(\operatorname{Hom}\left(\Lambda^{p}\left(T_{\mathcal{M}}\right) \otimes S\left(T_{\mathcal{M}}\right), \mathbb{K}\right)\right)
$$

and the total degree of $\omega \in \Omega^{p}\left(\widehat{S}\left(T_{\mathcal{M}}^{\vee}\right)\right)$ is $p+|\omega|$, where $p$ is the cohomological degree and $|\omega|$ is the internal degree.

Define two operators

and

$$
\delta: \Omega^{p}\left(\widehat{S}\left(T_{\mathcal{M}}^{\vee}\right)\right) \rightarrow \Omega^{p+1}\left(\widehat{S}\left(T_{\mathcal{M}}^{\vee}\right)\right)
$$

$\mathfrak{h}: \Omega^{p}\left(\widehat{S}\left(T_{\mathcal{M}}^{\vee}\right)\right) \rightarrow \Omega^{p-1}\left(\widehat{S}\left(T_{\mathcal{M}}^{\vee}\right)\right)$

by

$(\delta \omega)\left(X_{1} \wedge \cdots \wedge X_{p+1} ; Y_{1} \odot \cdots \odot Y_{q-1}\right)=\sum_{i=1}^{p+1}(-1)^{i+1} \varepsilon \cdot \omega\left(X_{1} \wedge \cdots \wedge \widehat{X}_{i} \wedge \cdots \wedge X_{p+1} ; X_{i} \odot Y_{1} \odot \cdots \odot Y_{q-1}\right)$ 
and

$(\mathfrak{h} \omega)\left(X_{1} \wedge \cdots \wedge X_{p-1} ; Y_{1} \odot \cdots \odot Y_{q+1}\right)=\frac{1}{p+q} \sum_{j=1}^{q+1} \varepsilon \cdot \omega\left(Y_{j} \wedge X_{1} \wedge \cdots \wedge X_{p-1} ; Y_{1} \odot \cdots \odot \widehat{Y}_{j} \odot \cdots \odot Y_{q+1}\right)$,

for all $\omega \in \Omega^{p}\left(\widehat{S}\left(T_{\mathcal{M}}^{\vee}\right)\right)$ and all homogeneous $X_{1}, \cdots, X_{p+1}, Y_{1}, \cdots, Y_{q+1} \in \mathfrak{X}(\mathcal{M})$. The symbol $\varepsilon$ denotes the Koszul signs: either $\varepsilon\left(X_{1}, \cdots, X_{p+1}, Y_{1}, \cdots, Y_{q-1}\right)$ or $\varepsilon\left(X_{1}, \cdots, X_{p-1}, Y_{1}, \cdots, Y_{q+1}\right)$, as appropriate.

Both $\delta$ and $\mathfrak{h}$ are $C^{\infty}(\mathcal{M})$-linear, and $\delta$ is the Koszul operator. Observe that $\delta$ has total degree +1 and $\mathfrak{h}$ has total degree -1 . However neither $\delta$ nor $\omega$ change the internal degree: $|\delta \omega|=|\omega|$ and $|\mathfrak{h} \omega|=|\omega|$ for $\omega \in \Omega^{p}\left(\widehat{S}\left(T_{\mathcal{M}}^{\vee}\right)\right)$.

Remark A.2. In $[18,19,32]$, the operator $\mathfrak{h}$ is written as $\delta^{-1}$. We avoid this notation because $\mathfrak{h}$ is not an inverse map of $\delta$, and it is rather a homotopy operator.

Lemma A.3. The operator $\delta$ satisfies $\delta^{2}=0$. That is,

$$
0 \rightarrow \Omega^{0}\left(\widehat{S}\left(T_{\mathcal{M}}^{\vee}\right)\right) \stackrel{\delta}{\rightarrow} \Omega^{1}\left(\widehat{S}\left(T_{\mathcal{M}}^{\vee}\right)\right) \stackrel{\delta}{\rightarrow} \Omega^{2}\left(\widehat{S}\left(T_{\mathcal{M}}^{\vee}\right)\right) \stackrel{\delta}{\rightarrow} \cdots
$$

forms a cochain complex. Moreover, it satisfies

$$
\delta \circ \mathfrak{h}+\mathfrak{h} \circ \delta=\mathrm{id}-\pi_{0}
$$

where $\pi_{0}: \Omega^{\bullet}\left(\widehat{S}\left(T_{\mathcal{M}}^{\vee}\right)\right) \rightarrow C^{\infty}(\mathcal{M})$ is the natural projection.

We have the following theorem

Theorem A.4 ([32, Theorem 5.6]). Let $\mathcal{M}$ be a finite dimensional graded manifold and $\nabla$ be a torsion-free affine connection on $\mathcal{M}$. Then the covariant derivative $d^{\nabla^{\xi}}$ decomposes as

$$
d^{\nabla^{\xi}}=d^{\nabla}-\delta+\widetilde{A^{\nabla}}
$$

where the operator $\widetilde{A^{\nabla}}: \Omega^{\bullet}\left(\widehat{S}\left(T_{\mathcal{M}}^{\vee}\right)\right) \rightarrow \Omega^{\bullet+1}\left(\widehat{S}\left(T_{\mathcal{M}}^{\vee}\right)\right)$, is a (total) degree +1 derivation determined by $A^{\nabla} \in \Omega^{1}\left(\mathcal{M}, \widehat{S}^{\geq 2}\left(T_{\mathcal{M}}^{\vee}\right) \otimes T_{\mathcal{M}}\right)$, satisfying

$$
\mathfrak{h} \circ A^{\nabla}=0 .
$$

Remark A.5. The operator $\widetilde{A^{\nabla}}$ increases the cohomological degree by +1 while it preserves the internal degree. That is, although the total degree of $\widetilde{A^{\nabla}}$ is +1 , we have the internal degree $\left|\widetilde{A^{\nabla}}\right|=0$

Write

$$
A^{\nabla}=\sum_{n \geq 2} A_{n}^{\nabla}, \quad A_{n}^{\nabla} \in \Omega^{1}\left(\mathcal{M}, S^{n}\left(T_{\mathcal{M}}^{\vee}\right) \otimes T_{\mathcal{M}}\right)
$$

Let $R^{\nabla} \in \Omega^{2}\left(\mathcal{M} ; \operatorname{End}\left(T_{\mathcal{M}}\right)\right)$ denote the curvature of $\nabla$.

Proposition A.6. We have the following recursive formula for $A_{n}^{\nabla}$ :

$$
\begin{gathered}
A_{2}^{\nabla}=\mathfrak{h} \circ R^{\nabla}, \\
A_{n+1}^{\nabla}=\mathfrak{h} \circ\left(d^{\nabla} A_{n}^{\nabla}+\sum_{p+q=n} \frac{1}{2}\left[A_{p}^{\nabla}, A_{q}^{\nabla}\right]\right), \quad \forall n \geq 2 .
\end{gathered}
$$

Proof. By Theorem A.4, the covariant derivative $d^{\nabla^{\xi}}=d^{\nabla}-\delta+A^{\nabla}$ and satisfies $\left(d^{\nabla^{\xi}}\right)^{2}=0$. 
By Lemma A.3, we know $\delta^{2}=0$ and $\delta \circ \mathfrak{h}+\mathfrak{h} \circ \delta=\mathrm{id}-\pi_{0}$. Also, $\left(d^{\nabla}\right)^{2}=R^{\nabla}$. Since $\nabla$ is torsion-free, we have

$$
\left[\delta, d^{\nabla}\right]=\delta \circ d^{\nabla}+d^{\nabla} \circ \delta=0 .
$$

As a result, $\left(d^{\nabla^{4}}\right)^{2}=0$ implies that

$$
\delta \circ A^{\nabla}+A^{\nabla} \circ \delta=R^{\nabla}+d^{\nabla} A^{\nabla}+\frac{1}{2}\left[A^{\nabla}, A^{\nabla}\right]
$$

By applying the operator $\mathfrak{h}$, we get

$$
A^{\nabla}=\mathfrak{h} \circ \delta \circ A^{\nabla}=\mathfrak{h} \circ\left(R^{\nabla}+d^{\nabla} A^{\nabla}+\frac{1}{2}\left[A^{\nabla}, A^{\nabla}\right]\right)
$$

because $\mathfrak{h} \circ A^{\nabla}=0$ and $\pi_{0} \circ A^{\nabla}=0$.

Since $\mathfrak{h}\left(\Omega^{2}\left(\widehat{S}^{q}\left(T_{\mathcal{M}}^{\vee}\right)\right) \subset \Omega^{1}\left(\widehat{S}^{q+1}\left(T_{\mathcal{M}}^{\vee}\right)\right)\right.$, applying the canonical projections

$$
\Omega^{1}\left(\mathcal{M}, \hat{S}\left(T_{\mathcal{M}}^{\vee}\right) \otimes T_{\mathcal{M}}\right) \rightarrow \Omega^{1}\left(\mathcal{M}, S^{n}\left(T_{\mathcal{M}}^{\vee}\right) \otimes T_{\mathcal{M}}\right)
$$

(for each $n \geq 2$ ) to the equality

$$
A^{\nabla}=\mathfrak{h} \circ\left(R^{\nabla}+d^{\nabla} A^{\nabla}+\frac{1}{2}\left[A^{\nabla}, A^{\nabla}\right]\right) \in \Omega^{1}\left(\mathcal{M}, \hat{S}\left(T_{\mathcal{M}}^{\vee}\right) \otimes T_{\mathcal{M}}\right)
$$

yields the relations

$$
\begin{gathered}
A_{2}^{\nabla}=\mathfrak{h} \circ R^{\nabla}, \\
A_{n+1}^{\nabla}=\mathfrak{h} \circ\left(d^{\nabla} A_{n}^{\nabla}+\sum_{p+q=n} \frac{1}{2}\left[A_{p}^{\nabla}, A_{q}^{\nabla}\right]\right), \quad \forall n \geq 2 .
\end{gathered}
$$

This completes the proof.

Corollary A.7. Under the same hypothesis as in Theorem A.4, the element $A_{n}^{\nabla} \in \Omega^{1}\left(\mathcal{M}, S^{n}\left(T_{\mathcal{M}}^{\vee}\right) \otimes T_{\mathcal{M}}\right)$, with $n \geq 2$, is completely determined by the curvature $R^{\nabla}$ and its higher covariant derivatives. In fact, $A_{n}^{\nabla}$ satisfies the recursive formula (67) involving $A_{k}^{\nabla}$, with $k \leq n-1$.

\section{ACKNOWLEDGMENTS}

We would like to thank Ruggero Bandiera, Camille Laurent-Gengoux, Hsuan-Yi Liao, Rajan Mehta and Luca Vitagliano for fruitful discussions and useful comments. Seokbong Seol is grateful to the Korea Institute for Advanced Study for its hospitality and generous support.

\section{REFERENCES}

[1] M. Alexandrov, A. Schwarz, O. Zaboronsky, and M. Kontsevich. "The geometry of the master equation and topological quantum field theory". In: Internat. J. Modern Phys. A 12.7 (1997), pp. 1405-1429. DOI: $10.1142 / \mathrm{S} 0217751 \mathrm{X} 97001031$.

[2] Ruggero Bandiera. "Formality of Kapranov's brackets in Kähler geometry via pre-Lie deformation theory". In: Int. Math. Res. Not. IMRN 21 (2016), pp. 6626-6655. DOI: 10.1093/imrn/rnv362.

[3] Ruggero Bandiera. "Homotopy abelian $L_{\infty}$ algebras and splitting property". In: Rend. Mat. Appl. (7) 37.1-2 (2016), pp. 105-122.

[4] Ruggero Bandiera, Zhuo Chen, Mathieu Stiénon, and Ping Xu. "Shifted derived Poisson manifolds associated with Lie pairs". In: Comm. Math. Phys. 375.3 (2020), pp. 1717-1760. DOI: 10.1007/s00220019-03457-w.

[5] Kai Behrend, Hsuan-Yi Liao, and Ping Xu. Derived Differentiable Manifolds. 2020. arXiv: 2006.01376 [math.DG].

[6] J. Michael Boardman. "The principle of signs". In: Enseign. Math. (2) 12 (1966), pp. 191-194. 
[7] Giuseppe Bonavolontà and Norbert Poncin. "On the category of Lie n-algebroids". In: J. Geom. Phys. 73 (2013), pp. 70-90. DOI: 10.1016/j.geomphys.2013.05.004.

[8] Andrew James Bruce. "From $L_{\infty}$-algebroids to higher Schouten/Poisson structures". In: Rep. Math. Phys. 67.2 (2011), pp. 157-177. DOI: 10.1016/S0034-4877(11)00010-3.

[9] Andrew James Bruce, Janusz Grabowski, and Luca Vitagliano. "Representations up to homotopy from weighted Lie algebroids". In: J. Lie Theory 28.3 (2018), pp. 715-737.

[10] Andrei Căldăraru and Simon Willerton. "The Mukai pairing. I. A categorical approach". In: New York J. Math. 16 (2010), pp. 61-98.

[11] David Carchedi and Dmitry Roytenberg. Homological Algebra for Superalgebras of Differentiable Functions. 2012. arXiv: 1212.3745 [math.AG].

[12] David Carchedi and Dmitry Roytenberg. "On theories of superalgebras of differentiable functions". In: Theory Appl. Categ. 28 (2013), No. 30, 1022-1098.

[13] Alberto S. Cattaneo and Florian Schätz. "Introduction to supergeometry". In: Rev. Math. Phys. 23.6 (2011), pp. 669-690. DOI: 10.1142/S0129055X11004400.

[14] Zhuo Chen, Mathieu Stiénon, and Ping Xu. "From Atiyah classes to homotopy Leibniz algebras". In: Comm. Math. Phys. 341.1 (2016), pp. 309-349. DOI: 10.1007/s00220-015-2494-6.

[15] Zhuo Chen, Maosong Xiang, and Ping Xu. "Atiyah and Todd classes arising from integrable distributions". In: J. Geom. Phys. 136 (2019), pp. 52-67. DOI: 10.1016/j.geomphys.2018.10.011.

[16] Zhuo Chen, Maosong Xiang, and Ping Xu. Hochschild cohomology of dg manifolds associated to integrable distributions. 2021. arXiv: 2103.08096 [math.DG].

[17] Jiahao Cheng, Zhuo Chen, and Dadi Ni. "Hopf algebras arising from dg manifolds". In: J. Algebra 584 (2021), pp. 19-68. DOI: 10.1016/j.jalgebra.2021.05.004.

[18] Vasiliy A. Dolgushev. "Covariant and equivariant formality theorems". In: Adv. Math. 191.1 (2005), pp. 147-177. DOI: 10.1016/j .aim. 2004.02.001.

[19] Claudio Emmrich and Alan Weinstein. "The differential geometry of Fedosov's quantization". In: Lie theory and geometry. Vol. 123. Progr. Math. Birkhäuser Boston, Boston, MA, 1994, pp. 217-239. DOI: 10.1007/978-1-4612-0261-5\_7.

[20] Katarzyna Grabowska and Janusz Grabowski. " $n$-tuple principal bundles". In: Int. J. Geom. Methods Mod. Phys. 15.12 (2018), pp. 1850211, 18. DOI: 10.1142/S0219887818502110.

[21] Janusz Grabowski. "Modular classes of skew algebroid relations". In: Transform. Groups 17.4 (2012), pp. 989-1010. DOI: $10.1007 / \mathrm{s} 00031-012-9197-2$.

[22] Madeleine Jotz Lean. "Lie 2-algebroids and matched pairs of 2-representations: a geometric approach". In: Pacific J. Math. 301.1 (2019), pp. 143-188. DOI: 10.2140/pjm.2019.301.143.

[23] Madeleine Jotz Lean, Rajan Amit Mehta, and Theocharis Papantonis. Modules and representations up to homotopy of Lie n-algebroids. 2020. arXiv: 2001.01101 [math.DG].

[24] Dominic Joyce. "An introduction to d-manifolds and derived differential geometry". In: Moduli spaces. Vol. 411. London Math. Soc. Lecture Note Ser. Cambridge Univ. Press, Cambridge, 2014, pp. 230-281.

[25] Mikhail Kapranov. "Rozansky-Witten invariants via Atiyah classes". In: Compositio Math. 115.1 (1999), pp. 71-113. DOI: 10.1023/A:1000664527238.

[26] Maxim Kontsevich. "Deformation quantization of Poisson manifolds". In: Lett. Math. Phys. 66.3 (2003), pp. 157-216. DOI: 10.1023/B:MATH.0000027508.00421.bf.

[27] Maxim Kontsevich. "Rozansky-Witten invariants via formal geometry". In: Compositio Math. 115.1 (1999), pp. 115-127. DOI: 10.1023/A:1000619911308.

[28] Alexei Kotov and Thomas Strobl. "Characteristic classes associated to Q-bundles". In: Int. J. Geom. Methods Mod. Phys. 12.1 (2015), pp. 1550006, 26. DOI: 10.1142/S0219887815500061.

[29] Tom Lada and Martin Markl. "Strongly homotopy Lie algebras". In: Comm. Algebra 23.6 (1995), pp. 2147-2161. DOI: $10.1080 / 00927879508825335$.

[30] Camille Laurent-Gengoux, Mathieu Stiénon, and Ping Xu. "Exponential map and $L_{\infty}$ algebra associated to a Lie pair". In: C. R. Math. Acad. Sci. Paris 350.17-18 (2012), pp. 817-821. DoI: 10.1016/j . crma.2012.08.009.

[31] Camille Laurent-Gengoux, Mathieu Stiénon, and Ping Xu. "Poincaré-Birkhoff-Witt isomorphisms and Kapranov dg-manifolds". In: Adv. Math. 387 (2021), Paper No. 107792, 62 pp. DOI: 10.1016/j . aim. 2021.107792. 
[32] Hsuan-Yi Liao and Mathieu Stiénon. "Formal exponential map for graded manifolds". In: Int. Math. Res. Not. IMRN 3 (2019), pp. 700-730. DOI: 10.1093/imrn/rnx130.

[33] Hsuan-Yi Liao, Mathieu Stiénon, and Ping Xu. "Formality theorem for differential graded manifolds". In: C. R. Math. Acad. Sci. Paris 356.1 (2018), pp. 27-43. DOI: 10.1016/j.crma.2017.11.017.

[34] S. L. Lyakhovich, E. A. Mosman, and A. A. Sharapov. "Characteristic classes of $Q$-manifolds: classification and applications". In: J. Geom. Phys. 60.5 (2010), pp. 729-759. DOI: 10.1016/j .geomphys . 2010.01 .008$.

[35] Marco Manetti. "On some formality criteria for DG-Lie algebras". In: J. Algebra 438 (2015), pp. 90118. DOI: $10.1016 / \mathrm{j} \cdot \mathrm{jalgebra.2015.04.029.}$

[36] Nikita Markarian. "The Atiyah class, Hochschild cohomology and the Riemann-Roch theorem". In: $J$. Lond. Math. Soc. (2) 79.1 (2009), pp. 129-143. DOI: 10.1112/jlms/jdn064.

[37] Rajan A. Mehta. "Q-algebroids and their cohomology". In: J. Symplectic Geom. 7.3 (2009), pp. 263293.

[38] Rajan A. Mehta, Mathieu Stiénon, and Ping Xu. "The Atiyah class of a dg-vector bundle". In: C. R. Math. Acad. Sci. Paris 353.4 (2015), pp. 357-362. DOI: 10.1016/j.crma.2015.01.019.

[39] Rajan Amit Mehta. Supergroupoids, double structures, and equivariant cohomology. 2006. arXiv: math/ 0605356 [math.DG].

[40] J. P. Pridham. "A differential graded model for derived analytic geometry". In: Adv. Math. 360 (2020), pp. 106922, 29. DOI: 10.1016/j.aim.2019.106922.

[41] J. P. Pridham. An outline of shifted Poisson structures and deformation quantisation in derived differential geometry. 2018. arXiv: 1804.07622 [math.DG].

[42] Ajay C. Ramadoss. "The big Chern classes and the Chern character". In: Internat. J. Math. 19.6 (2008), pp. 699-746. DOI: 10.1142/S0129167X08004856.

[43] Justin Roberts and Simon Willerton. "On the Rozansky-Witten weight systems". In: Algebr. Geom. Topol. 10.3 (2010), pp. 1455-1519. DOI: 10.2140/agt.2010.10.1455.

[44] Dmitry Roytenberg. "On the structure of graded symplectic supermanifolds and Courant algebroids". In: Quantization, Poisson brackets and beyond (Manchester, 2001). Vol. 315. Contemp. Math. Amer. Math. Soc., Providence, RI, 2002, pp. 169-185. DOI: 10.1090/conm/315/05479.

[45] Justin Sawon. Rozansky-Witten invariants of hyperkähler manifolds. Thesis (Ph.D.)-University of Cambridge. 2000. arXiv: math/0404360 [math.DG].

[46] Albert Schwarz. "Geometry of Batalin-Vilkovisky quantization". In: Comm. Math. Phys. 155.2 (1993), pp. 249-260.

[47] Pavol Ševera. Letters to Alan Weinstein about Courant algebroids. 2017. arXiv: 1707.00265 [math.DG].

[48] Pavol Ševera. "Some title containing the words "homotopy" and "symplectic", e.g. this one". In: Travaux mathématiques. Fasc. XVI. Vol. 16. Trav. Math. Univ. Luxemb., Luxembourg, 2005, pp. 121-137.

[49] Boris Shoikhet. On the Duflo formula for $L_{\infty}$-algebras and Q-manifolds. 1998. arXiv: math/9812009 [math.QA].

[50] David I. Spivak. "Derived smooth manifolds". In: Duke Math. J. 153.1 (2010), pp. 55-128. DoI: 10. 1215/00127094-2010-021.

[51] Mathieu Stiénon and Ping Xu. "Atiyah classes and Kontsevich-Duflo type theorem for DG manifolds". In: Homotopy algebras, deformation theory and quantization. Vol. 123. Banach Center Publ. Polish Acad. Sci. Inst. Math., Warsaw, 2021, pp. 63-110. DOI: 10.4064/bc123-3.

[52] Luca Vitagliano. "On the strong homotopy associative algebra of a foliation". In: Commun. Contemp. Math. 17.2 (2015), pp. 1450026, 34. DOI: 10.1142/S0219199714500266.

[53] Yannick Voglaire and Ping Xu. "Rozansky-Witten-type invariants from symplectic Lie pairs". In: Comm. Math. Phys. 336.1 (2015), pp. 217-241. DOI: 10.1007/s00220-014-2221-8.

[54] Theodore Th. Voronov. "Q-manifolds and higher analogs of Lie algebroids". In: XXIX Workshop on Geometric Methods in Physics. Vol. 1307. AIP Conf. Proc. Amer. Inst. Phys., Melville, NY, 2010, pp. 191-202.

[55] Theodore Th. Voronov. "Q-manifolds and Mackenzie theory". In: Comm. Math. Phys. 315.2 (2012), pp. 279-310. DOI: $10.1007 / \mathrm{s} 00220-012-1568-\mathrm{y}$.

[56] Theodore Th. Voronov. "Graded Geometry, Q-Manifolds, and Microformal Geometry". In: Fortschritte der Physik 67.8-9 (May 2019), p. 1910023. DOI: 10.1002/prop. 201910023. 
[57] Kentaro Yano and Shigeru Ishihara. Tangent and cotangent bundles: differential geometry. Pure and Applied Mathematics, No. 16. Marcel Dekker, Inc., New York, 1973, pp. ix+423.

Department of Mathematics, Pennsylvania State University

Email address: sxs803@psu.edu

Department of Mathematics, Pennsylvania State University

Email address: stienon@psu.edu

Department of Mathematics, Pennsylvania State University

Email address: ping@math.psu.edu 